\title{
Error-analysis and comparison to analytical models of numerical waveforms produced by the NRAR Collaboration
}

\author{
Ian Hinder ${ }^{1}$, Alessandra Buonanno ${ }^{2}$, Michael Boyle ${ }^{3}$, \\ Zachariah B. Etienne $^{4}$, James Healy ${ }^{5}$, Nathan K. \\ Johnson-McDaniel ${ }^{6}$, Alessandro Nagar ${ }^{7}$, Hiroyuki \\ Nakano $^{8,9}$, Yi Pan $^{2}$, Harald P. Pfeiffer ${ }^{10,11}$, Michael \\ Pürrer $^{12}$, Christian Reisswig ${ }^{13}$, Mark A. Scheel ${ }^{13}$, Erik \\ Schnetter $^{14,15,16}$, Ulrich Sperhake ${ }^{13,17,18,19}$, Bela Szilágyi ${ }^{13}$, \\ Wolfgang Tichy ${ }^{20}$, Barry Wardell ${ }^{1,21}$, Anıl Zenginoğlu ${ }^{13}$, \\ Daniela Alic ${ }^{1}$, Sebastiano Bernuzzi ${ }^{6}$, Tanja Bode ${ }^{5}$, Bernd \\ Brügmann $^{6}$, Luisa T. Buchman ${ }^{13}$, Manuela Campanelli ${ }^{8}$, \\ Tony Chu ${ }^{10,13}$, Thibault Damour ${ }^{8}$, Jason D. Grigsby ${ }^{6}$, Mark \\ Hannam $^{12}$, Roland Haas ${ }^{5,13}$, Daniel A. Hemberger ${ }^{3,13}$, \\ Sascha Husa ${ }^{26}$, Lawrence E. Kidder ${ }^{3}$, Pablo Laguna ${ }^{5}$, \\ Lionel London ${ }^{5}$, Geoffrey Lovelace ${ }^{3,13,22}$, Carlos O. Lousto ${ }^{8}$, \\ Pedro Marronetti ${ }^{20,23}$, Richard A. Matzner ${ }^{24}$, Philipp \\ Mösta $^{1,13}$, Abdul Mroué ${ }^{10}$, Doreen Müller ${ }^{6}$, Bruno C. \\ Mundim ${ }^{1,8}$, Andrea Nerozzi ${ }^{25}$, Vasileios Paschalidis ${ }^{4}$, Denis \\ Pollney $^{26,27}$, George Reifenberger ${ }^{20}$, Luciano Rezzolla ${ }^{1,29}$, \\ Stuart L. Shapiro ${ }^{4,28}$, Deirdre Shoemaker ${ }^{5}$, Andrea \\ Taracchini $^{2}$, Nicholas W. Taylor ${ }^{13}$, Saul A. Teukolsky ${ }^{3}$, \\ Marcus Thierfelder ${ }^{6}$, Helvi Witek ${ }^{17,25}$, Yosef Zlochower ${ }^{8}$ \\ ${ }^{1}$ Max-Planck-Institut für Gravitationsphysik, Albert-Einstein-Institut, Am \\ Mühlenberg 1, D-14476 Golm, Germany \\ 2 Maryland Center for Fundamental Physics \& Joint Space-Science Institute, \\ Department of Physics, University of Maryland, College Park, MD 20742, USA \\ 3 Center for Radiophysics and Space Research, Cornell University, Ithaca, New \\ York 14853, USA \\ ${ }^{4}$ Department of Physics, University of Illinois at Urbana-Champaign, Urbana, \\ IL 61801 \\ ${ }^{5}$ Center for Relativistic Astrophysics, School of Physics, Georgia Institute of \\ Technology, Atlanta, GA 30332-0430 \\ 6 Theoretisch-Physikalisches Institut, Friedrich-Schiller-Universität, \\ Max-Wien-Platz 1, 07743 Jena, Germany \\ 7 Institut des Hautes Études Scientifiques, 91440 Bures-sur-Yvette, France \\ 8 Center for Computational Relativity and Gravitation, and School of \\ Mathematical Sciences, Rochester Institute of Technology, 85 Lomb Memorial \\ Drive, Rochester, New York 14623 \\ 9 Yukawa Institute for Theoretical Physics, Kyoto University, Kyoto, 606-8502, \\ Japan \\ 10 Canadian Institute for Theoretical Astrophysics, University of Toronto, \\ Toronto, Ontario M5S 3H8, Canada \\ ${ }^{11}$ Canadian Institute for Advanced Research, 180 Dundas Street West, Suite \\ 1400, Toronto, Ontario M5G 1Z8, Canada \\ 12 School of Physics and Astronomy, Cardiff University, Queens Building, CF24 \\ 3AA, Cardiff, United Kingdom
}


13 Theoretical Astrophysics 350-17, California Institute of Technology, Pasadena, CA 91125

${ }^{14}$ Perimeter Institute for Theoretical Physics, Waterloo, ON N2L 2Y5, Canada

15 Department of Physics, University of Guelph, Guelph, ON N1G 2W1, Canada

${ }^{16}$ Center for Computation \& Technology, Louisiana State University, Baton Rouge, LA 70803, USA

17 Department of Applied Mathematics and Theoretical Physics, Centre for Mathematical Sciences, University of Cambridge, Wilberforce Road, Cambridge CB3 0WA, United Kingdom

18 Institute of Space Sciences, CSIC-IEEC, 08193 Bellaterra, Spain

19 Department of Physics and Astronomy, The University of Mississippi, University, MS 38677, USA

20 Department of Physics, Florida Atlantic University, Boca Raton, FL 33431, USA

${ }^{21}$ School of Mathematical Sciences and Complex \& Adaptive Systems

Laboratory, University College Dublin, Belfield, Dublin 4, Ireland

${ }^{22}$ Gravitational Wave Physics and Astronomy Center, California State University Fullerton, Fullerton, California 92834, USA

23 Division of Physics, National Science Foundation, Arlington, VA 22230, USA

24 Center for Relativity, Department of Physics, The University of Texas at Austin, Austin, TX 78712

${ }_{25}$ CENTRA, Departamento de Física, Instituto Superior Técnico, Universidade Técnica de Lisboa - UTL, Av. Rovisco Pais 1, 1049 Lisboa, Portugal

${ }^{26}$ Departament de Física, Universitat de les Illes Balears, Palma de Mallorca, E-07122 Spain

27 Department of Mathematics, Rhodes University, Grahamstown, 6139 South Africa

28 Department of Astronomy and NCSA, University of Illinois at Urbana-Champaign, Urbana, IL 61801

${ }^{29}$ Institut für Theoretische Physik, Max-von-Laue-Str. 1, D-60438 Frankfurt am Main, Germany

E-mail: ian.hinder@aei.mpg.de

Abstract. The Numerical-Relativity-Analytical-Relativity (NRAR) collaboration is a joint effort between members of the numerical relativity, analytical relativity and gravitational-wave data analysis communities. The goal of the NRAR collaboration is to produce numerical-relativity simulations of compact binaries and use them to develop accurate analytical templates for the LIGO/Virgo Collaboration to use in detecting gravitational-wave signals and extracting astrophysical information from them. We describe the results of the first stage of the NRAR project, which focused on producing an initial set of numerical waveforms from binary black holes with moderate mass ratios and spins, as well as one non-spinning binary configuration which has a mass ratio of 10 . All of the numerical waveforms are analysed in a uniform and consistent manner, with numerical errors evaluated using an analysis code created by members of the NRAR collaboration. We compare previously-calibrated, non-precessing analytical waveforms, notably the effective-one-body (EOB) and phenomenological template families, to the newly-produced numerical waveforms. We find that when the binary's total mass is $\sim 100-200 M_{\odot}$, current EOB and phenomenological models of spinning, non-precessing binary waveforms have overlaps above $99 \%$ (for advanced LIGO) with all of the non-precessing-binary numerical waveforms with mass ratios $\leq 4$, when maximizing over binary parameters. This implies that the loss of event rate due to modelling error is below $3 \%$. Moreover, the non-spinning EOB waveforms previously calibrated to five non-spinning waveforms with mass ratio smaller than 6 have overlaps above $99.7 \%$ with the numerical waveform with a mass ratio of 10 , without even maximizing on the binary parameters.

PACS numbers: 04.25.dg, 04.30.-w, 04.25.D-, 04.25.-g 


\section{Introduction}

A worldwide network of interferometric gravitational-wave detectors has been operating since 2002. This network includes the three LIGO detectors [1] in the United States, the French-Italian Virgo detector 2 in Italy, and the British-German GEO600 detector 3] in Germany. After five years of collecting and analysing data (2005-2010), the LIGO and Virgo detectors were temporarily shut down and are currently being upgraded to the advanced LIGO and Virgo configurations [4. These upgrades will improve the detector sensitivities by a factor of 10 . As a consequence, event rates for coalescing binary systems will increase by a factor of one thousand, very likely leading to the first detection and establishing the field of gravitational-wave astronomy 5 .

The upgrades to the advanced interferometric configurations are expected to be complete in 2015, with Advanced LIGO expected to reach full design sensitivity around 2018-2019, although several month-long periods of observations are planned to take place as early as 2015 6. Furthermore, an underground cryogenic detector in Japan known as KAGRA is under construction [7, and there are plans for one of the advanced LIGO detectors to be built in India to improve sky localization 6, 8. During this time of upgrades and construction, the GEO600 detector continues to operate in the Astrowatch program to capture any potential strong events, such as a supernova in our galaxy. Finally, efforts to build a gravitational-wave detector in space are under way $[9,10$.

Binary systems of compact objects (compact binaries for short), composed of black holes and/or neutron stars, are among the most promising sources for gravitationalwave detectors. For this class of gravitational-wave sources, signal detection and interpretation are based on the method of matched filtering, where the noisy detector output is cross-correlated with a bank of theoretical templates [11 19]. A detailed and accurate understanding of the gravitational waves radiated as the bodies in a binary spiral towards each other is crucial not only for the initial detection of such sources, but also for maximizing the information that can be obtained from the gravitational-wave signals once they are observed.

The frequency bandwidth of ground-based detectors is $\sim 10-10^{3} \mathrm{~Hz}$, with best sensitivity in the $\sim 100-200 \mathrm{~Hz}$ frequency range. Binary neutron stars, having masses $\sim 1-3 M_{\odot}$, are expected to accumulate the majority of the signal-to-noise ratio (SNR) during the inspiraft with the merger at frequencies $\gtrsim 1 \mathrm{kHz}$. In this case, the gravitational waveform can be computed quite accurately using the post-Newtonian $(\mathrm{PN})$ approach that expands the Einstein equations in the ratio of the characteristic velocity of the binary $v$ to the speed of light 20]. For instance, the most sensitive search for gravitational waves from binary neutron stars with the LIGO and Virgo detectors 21 employed non-spinning inspiral templates computed at 3.5PN order, i.e. $(v / c)^{7}$ 过22 25, which were shown [26, 27] to be sufficient for searches of nonspinning compact object binaries of total mass up to $12 M_{\odot}$.

As the total mass of the binary increases, the frequencies during late inspiral, merger, and ringdown decrease and move into the most sensitive frequency range of the detectors. First, the late inspiral becomes important. Post-Newtonian waveforms become inaccurate in this regime where $v / c$ approaches unity, as investigated in a

$\dagger$ As a rule of thumb, an estimate of the gravitational-wave (GW) frequency at which the inspiral ends can be obtained from the Schwarzschild innermost-stable circular orbit, and is given by $f_{\mathrm{GW}} \simeq 4400 /\left(M / M_{\odot}\right) \mathrm{Hz}, M$ being the total mass of the binary.

$\ddagger$ Powers of $(v / c)^{n}$ correspond to $(n / 2)$ PN order with respect to the leading Newtonian term. 
series of comparisons against numerical-relativity results 28 35], and care must be taken to develop and employ waveform templates with the correct phasing. As the mass increases further, the merger and eventually (at masses of a few hundred solar masses) the ringdown of the final black hole move into the most sensitive frequency range. The late-inspiral and merger phases are also the most energetic parts of the binary evolution, where up to $11 \%$ of the initial total mass of the binary is radiated in gravitational waves 36]. Such high-mass binary systems composed of black holes extend the horizon distance of advanced LIGO and Virgo detectors from $\sim 450 \mathrm{Mpc}$ (for binary neutron stars) to $\sim 1-20 \mathrm{Gpc}$ depending on the binary's total mass, massratio and spin§. To detect binary black holes effectively and to take full advantage of the discovery potential of the detectors, it is crucial to use template banks built from complete and accurate inspiral-merger-ringdown waveform-models. This requires an accurate description of the non-linear, strong-field stages of binary evolution, best provided by numerical relativity simulations.

After the dazzling breakthroughs in 2005 [37 39], today several groups are able to simulate on supercomputers the merger of compact binaries composed of black holes and/or neutron stars (for reviews, see e.g. 40 46]). Important recent advances include simulations of black hole binaries with precession [33, 47, 55], large spins 36, 56, 59, large mass ratios 60, 61, large initial separations [62 and large recoils 63 65], as well as particularly long and accurate simulations [35, 58, 66 and simulations in the scalar-tensor 67 69 and $f(R)$ 70 theories of gravity. However, due to the high computational cost of numerical simulations, template construction is currently not possible with numerical-relativity simulations alone.

Motivated by the construction of LIGO and Virgo detectors, an analytical approach that combines the PN expansion and perturbation theory, known as the effective-one-body (EOB) approach, was introduced $71-73$. This novel approach was aimed at modelling the plunge, merger and ringdown signal of comparablemass black holes using physically-motivated guesses, analogies to the test-particle limit 74 76] and insights from the close-limit approximation [77]. The EOB approach incorporates nonperturbative and strong-field effects that are lost when the dynamics and the waveforms are Taylor-expanded as PN series. Several predictions of the EOB approach, notably the simplicity of the merger signal for non-spinning 72 and spinning, precessing black holes [78, have been confirmed by the results of numericalrelativity simulations. The EOB waveforms have been improved over the years, being calibrated to progressively more accurate numerical-relativity waveforms [31,79 88 .

A second class of phenomenological inspiral-merger-ringdown waveform models has also been developed, starting with 8990 . In this case, the original motivation was to provide LIGO and Virgo detectors with inspiral, merger and ringdown waveforms that could be computed efficiently during searches and be used to observe high-mass binary black holes. The procedure has proved sufficiently flexible and attractive that it was also used to construct the first inspiral-merger-ringdown models of non-precessing binaries calibrated to numerical-relativity waveforms 91, 92. The phenomenological waveforms were constructed by first matching inspiral PN templates and numericalrelativity waveforms in either the time or frequency domain, and then fitting this hybrid waveform in the frequency domain to a stationary phase approximation based template augmented by a Lorentzian function for the ringdown stage. The first

$\S$ The horizon distance is the maximum distance at which advanced LIGO and Virgo can claim a detection for an optimally oriented binary. We compute the horizon distances at a single-detector SNR of 8 . 
searches for gravitational waves from non-spinning high-mass binary black holes with the LIGO and Virgo detectors 93 95] employed EOB templates calibrated to numerical-relativity waveforms to filter the data, while phenomenological templates were used as injection templates to study the efficiency of the search algorithm, and have been used in LIGO-Virgo parameter estimation studies [96].

All these important numerical and analytical advances have brought us closer to the goal of observing and interpreting gravitational waves from compact binaries. However, formidable challenges remain because large portions of the binary parameter space are not yet covered by accurate templates. An efficient way to span the entire parameter space and build accurate waveforms for LIGO and Virgo searches is to coordinate the efforts among the numerical-relativity groups and plan simulations together with the analytical-relativity and gravitational-wave astrophysics communities. This is the main motivation that led to the formation of the NumericalRelativity-Analytical-Relativity (NRAR) collaboration in early 2010. To this end, the U.S. National Science Foundation (NSF) made available to the NRAR collaboration 11 million CPU hours on the Teragrid machine Kraken. The complementary Numerical INJection Analysis (NINJA) project was created in 2008 97 99. NINJA brings together the numerical-relativity and data-analysis communities with the goal of testing the LIGO and Virgo analysis pipelines by adding physically realistic signals, i.e. the numerical-relativity waveforms, to the detector noise in software. When pursuing these tests, analytical template banks based on PN, EOB and phenomenological waveforms, which are available in the LIGO and Virgo software, are used to recover the injected signals. Those analytical template banks are the ones we aim to improve in the NRAR collaboration.

In this first paper, we produce an initial set of numerical waveforms from binary black holes with moderate mass ratios and spins, as well as one non-spinning binary configuration which has a mass ratio of 10 . We provide a comprehensive technical review of current black-hole-binary simulation codes and methods. We evaluate the numerical errors in a uniform and consistent manner, and then compare the numerical waveforms to previously-calibrated analytical waveforms to test their robustness and understand whether they need to be improved to better match the numerical waveforms.

In this work, we compare only the $\ell=2, m=2$ mode of the NR waveforms and analytical models, though other modes are available in the NR data. Since most of the energy is radiated in the $\ell=2, m=2$ mode, most analytical modelling work has focused on this mode, and it is the only mode that has been considered so far in searches for compact binaries. However, several studies have investigated the effects of other modes on gravitational wave detection algorithms $27,54,86$. Since there can be a significant mismatch between waveforms that include other modes and waveforms that only include the dominant mode, these modes will likely need to be calibrated in analytical models in the future.

The paper is organized as follows. We start in Sec. 2 discussing the importance of modelling the late inspiral, merger and ringdown phases of the binary coalescence when searching for gravitational waves with advanced LIGO and Virgo detectors. In Sec. 3 we discuss the scientific plan of the NRAR collaboration, explaining how we selected which numerical-relativity simulations to perform from the binary parameter space. We also review the requirements on the waveforms' length and the accuracy requirements on the waveforms' phase and amplitude. In Sec. 4, we discuss the numerical codes that were used to carry out the simulations, and provide 
a comprehensive review of current methods. In Sec. 5 we analyse the waveforms and compute the numerical errors. This is the most comprehensive error analysis to date that was applied consistently to waveforms produced with different numericalrelativity codes. Whereas the goal of previous code-comparison studies 100,101] was to compare simulations of identical binary configurations, here we consider only one configuration simulated by two codes, and focus instead on a uniform analysis of resolution and waveform extraction uncertainties across 25 waveforms produced by 9 groups using 7 numerical-relativity codes. In Sec. 6 we investigate how existing analytical waveforms match the numerical waveforms produced in this paper. Finally, Sec. 7 summarizes our main results and gives recommendations for future projects within the NRAR collaboration.

\section{Relevance of late-inspiral, merger and ringdown phases for advanced LIGO and Virgo searches}

To illustrate the importance of the late inspiral and merger, figure 1 shows a waveform for a binary black hole system with total mass $M=30 M_{\odot}$, mass ratio $q=3$ and dimensionless spins $\chi_{1}=-0.6, \chi_{2}=0$, where the minus sign indicates that the spin is oriented anti-parallel to the system's orbital angular momentum; we shall follow this sign convention in all non-precessing cases in the paper. The figure also includes the waveform whitened by the square-root of the zero-detune high power noise spectral density of the advanced LIGO detector 102. The waveform parameters agree with one of the numerical waveforms produced in the NRAR collaboration (Case 24 in table 11. The vertical lines mark 10\% intervals for accumulated SNR. For this case, the last 20 gravitational-wave cycles contribute $>50 \%$ of the total SNR. Therefore, for total masses $M \gtrsim 30 M_{\odot}$, the late inspiral, merger and ringdown waveform is crucial for detecting the signal, and in the absence of numerical and analytical modelling, a significant fraction of the SNR will be lost. In fact, the systematic study in 26] suggests that ideally inspiral-merger-ringdown waveforms would be used in searches for binaries with $M \gtrsim 12 M_{\odot}$.

Figure 1 also demonstrates that a significant portion of the SNR is accumulated well before merger; in this case, $40 \%$ of the SNR is accumulated before the last 30 cycles. NR simulations often last for only 20-30 gravitational-wave cycles, and therefore they alone will also not be sufficient to provide templates for LIGO and Virgo detectors. The gravitational-wave frequency at the start of the NR simulation provides an intuitive way to think about length requirements as a function of total mass. Numerical simulations can be rescaled to any total mass; when doing so, their dimensionless orbital frequency $M \Omega_{\text {in }}$ at the start of the waveform is mapped to a gravitational-wave frequency

$$
f_{\mathrm{GW}, \text { in }} \sim 13 \mathrm{~Hz} \frac{M \Omega_{\mathrm{in}}}{0.02}\left(\frac{M}{100 M_{\odot}}\right)^{-1} .
$$

The higher the mass $M$ of the binary, the lower this frequency. For a rather typical $M \Omega_{\text {in }} \sim 0.02$ (20-30 gravitational-wave cycles before merger), 11 indicates that the numerical waveform will cover the entire advanced LIGO frequency band only for $M \gtrsim 100 M_{\odot}$.

To better understand how numerical-relativity waveforms and the analytical modelling are crucial to either detecting or improving the searches for gravitational 
$t$ [sec]

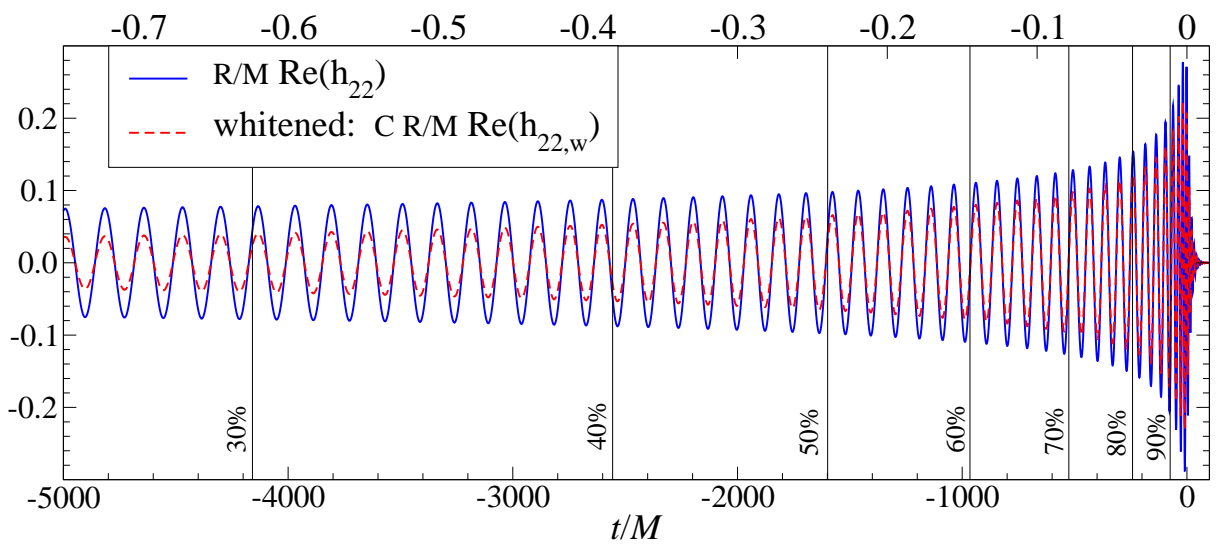

Figure 1. The $(\ell, m)=(2,2)$ mode of a binary black hole waveform with $M=30 M_{\odot}$, mass ratio $q=3$ and dimensionless spins $\chi_{1}=-0.6, \chi_{2}=0$. Also shown is the same signal whitened by the square-root of the noise spectral density of the advanced LIGO detector, and multiplied by $C=3.1 \times 10^{-24}$ for plotting on the same scale. Shown is a time-domain EOB waveform model with parameters that agree with one of the numerical waveforms presented in this paper (Case 24 $(\mathrm{S} 3-60+00)$ in table 1). The vertical lines mark $10 \%$ intervals of accumulated SNR, and are labelled by the fraction of SNR accumulated before each line.

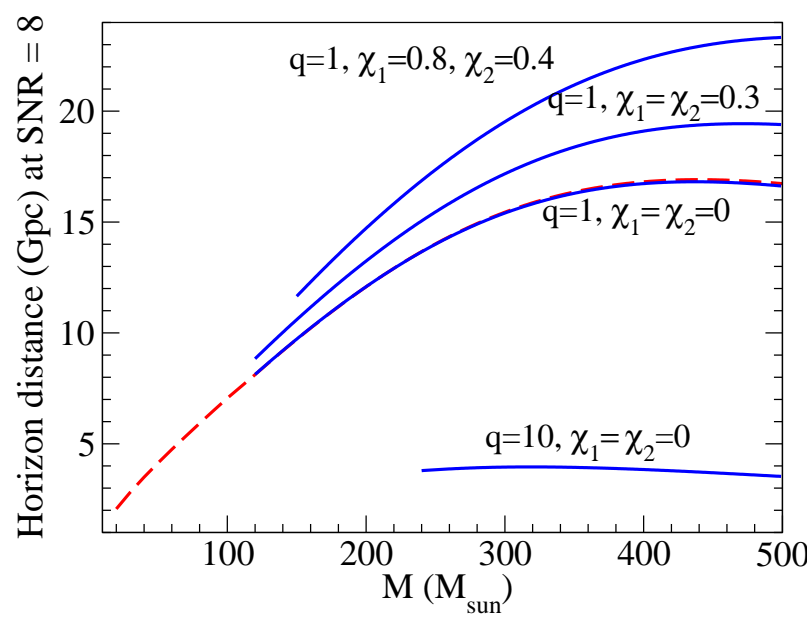

Figure 2. We show the horizon distance versus the total (redshifted) mass using some of the numerical-relativity waveforms produced in this paper (Cases 15 $(\mathrm{P} 1+80+40), 18(\mathrm{~S} 1+30+30)$ and $9(\mathrm{R} 10)$; cf. table 1). For mass ratio $q=1$ and no spin, we also show the numerical-relativity waveform of 103 (solid line) and the EOB calibrated waveform of [86] (dashed line). The numerical lines are shown only for those masses where the numerical waveform starts at frequency $10 \mathrm{~Hz}$ or below. 
waves from binary black holes, we plot in figure 2 the horizon distance, setting the single-detector SNR to 8, versus the total (redshifted) mass, for a few binary mass ratios and spins. The curves are obtained using the numerical-relativity waveforms produced in the NRAR collaboration. We also show the $q=1$ non-spinning numericalrelativity waveform of [103] and the EOB calibrated waveform of [86]. In figure 2, the horizon-distance curves are computed only for those total masses where numerical waveform starts at $\leq 10 \mathrm{~Hz}$, i.e. for which it covers the detector bandwidth. For the case $q=1$, the EOB curve extends to lower masses because the EOB waveform can easily be computed starting at lower orbital frequencies. Thus, unless numericalrelativity waveforms are computed starting at much lower frequencies, the searches covering total masses $M \lesssim 130 M_{\odot}$ have to rely (in part) on analytical waveforms. The latter have to accurately model both the merger and the last tens or hundreds of inspiralling cycles. Longer numerical-relativity waveforms are then crucial in testing the robustness of those analytical waveforms.

\section{Selecting numerical-relativity simulations}

\subsection{Spanning the binary parameter space}

The scientific plan of the NRAR collaboration was set up in early 2010. Considering the results that were available at the time on analytical waveforms calibrated to numerical-relativity waveforms in the non-spinning and spinning, non-precessing cases $31,80,85,90,92,104$ and the limited set of numerical-relativity waveforms in the spinning case, we proposed a general plan to span the parameter space that will (i) underpin an initial version of the analytical model for spinning binary systems, (ii) identify regions of parameter space where the spinning waveforms are so sensitive to changes of parameters that they will require further simulations, (iii) provide detailed input for the design of those further simulations, and (iv) provide an estimate of how many further simulations will be required to provide analytical templates to be used for detection (and later for parameter estimation) by the advanced LIGO and Virgo detectors. As we shall discuss below, we compare previously-calibrated analytical waveforms to the initial set of numerical waveforms produced by the NRAR collaboration, and find that current non-precessing EOB and phenomenological templates match sufficiently well binary systems with mild spins and mass ratios. Thus, future effort should focus on producing simulations of binary systems with larger spins and mass ratios, and stronger spin-induced precessional modulations.

A generic gravitational waveform emitted by a binary system of black holes with spin is described by 7 parameters: the mass ratio $q \equiv m_{1} / m_{2} \geq 1$ and the components of the spin vectors $\boldsymbol{S}_{1}$ and $\boldsymbol{S}_{2}$ at some initial time. (The spin vectors are related to the dimensionless spins $\chi_{1}$ and $\chi_{2}$ by $\left|\mathbf{S}_{1}\right| \equiv m_{1}^{2} \chi_{1}$ and $\left|\mathbf{S}_{2}\right| \equiv m_{2}^{2} \chi_{2}$. $\mathbf{~ T h e ~ t o t a l ~}$ mass of the system, $M=m_{1}+m_{2}$, provides an overall scaling, and is therefore not part of the parameter space of binary configurations that need to be simulated. We also assume that the system has been circularized by gravitational radiation during a lengthy inspiral, so its eccentricity is negligible.) For black holes moving along an adiabatic sequence of inspiralling, circular orbits, up to very close to merger, these 7 parameters reduce to 6 , since the initial spin vectors $\boldsymbol{S}_{1}$ and $\boldsymbol{S}_{2}$ can rotate together around the initial, orbital angular-momentum vector without changing the waveform.

ๆIn the non-precessing, aligned-spin cases, we abuse notation somewhat and give $\chi_{1,2}$ a sign indicating whether the spin is aligned $(+)$ or anti-aligned $(-)$ with the orbital angular momentum. 
At first sight, given the large number of parameters, it seems an ambitious goal to build an accurate analytical model that covers the entire parameter space. In fact, a naive calculation which does not take into account any degeneracy of the parameter space and any hints from the analytical spin modelling leads immediately to a very large number of simulations required to span the parameter space - for example, we obtain $5^{6} \approx 15000$ simulations for merely five sample values in each binary parameter's dimension.

However, exploiting degeneracies of the parameter space, a rough estimation concludes that we need only a few hundred simulations to achieve the goal of building an analytical model accurate enough for detection. One argument goes as follows. Within the accuracy requirement for detection, in roughly $97 \%$ of the binary parameter space, the PN inspiral waveforms are the same as one would get with a suitablychosen single-effective-spin black-hole binary [II [105 107]. This implies that the parameter space's dimensionality is effectively reduced from 6 to 2 (non-precessing) or 3 (precessing) 105 107 ** — at least for the inspiral waveforms. Based on experience with both the one-dimensional non-spinning case $80,86,88,90$ and nonprecessing cases 87 91 92 109, we expect that in each parameter space's dimension $\sim 5$ numerical-relativity waveforms will be sufficient. The feasibility of producing a nonprecessing-binary model using either a handful of non-precessing waveforms in the small mass ratio limit 109 and only two waveforms in the equal-mass case 85, 87, or $\sim 5^{2}=25$ waveforms in the comparable mass case, has been demonstrated in the EOB and phenomenological models in Refs. 85, 87, 91, 92. This then suggests that most of the precessing parameter space might be covered by roughly $5^{3}=125$ inspiral waveforms. We may increase the number of simulations to compare and validate numerical-relativity waveforms produced by different codes. We may also include several simulations to test the degeneracy of the parameter space predicted within the PN description of inspiral waveforms, which went into our counting argument above, for example as done recently in 110. Furthermore, we may need to add a certain number of merger waveforms, because the degeneracy during the inspiral may break in the non-linear, highly relativistic phase. However, short merger waveforms, e.g. less than 10 gravitational-wave cycles, are much cheaper to simulate numerically than lengthy inspirals. Finally, for the ringdown waveforms, results of numerical simulations and symmetry arguments suggest that the mass and spin of the final black hole, and hence ringdown frequencies and quality factors, can be estimated well from the premerger configuration using simple analytical formulas [111 115].

The above considerations lead us to conclude that an initial survey of about 200 numerical simulations may be sufficient for detection purposes. However, at the time the NRAR scientific plan was established, the simulation of a few hundred configurations were still a demanding task for numerical relativity. Thus, we decided to proceed in steps, dividing our initial survey into three stages - starting with less challenging simulations in the first stage, extending to more generic simulations in the second stage and concluding with several more challenging simulations in the third stage.

In the first stage, we planned 58 non-precessing and 19 precessing simulations.

|lThe remaining $3 \%$ of parameter space, which stays six-dimensional in the adiabatic approximation, has at least one dimensionless spin larger than 0.7 and mass ratios $q$ between about 2 and 3 [105.

**We note that for some binary configurations the single effective spin can be described by only 2 parameters 107108 . 


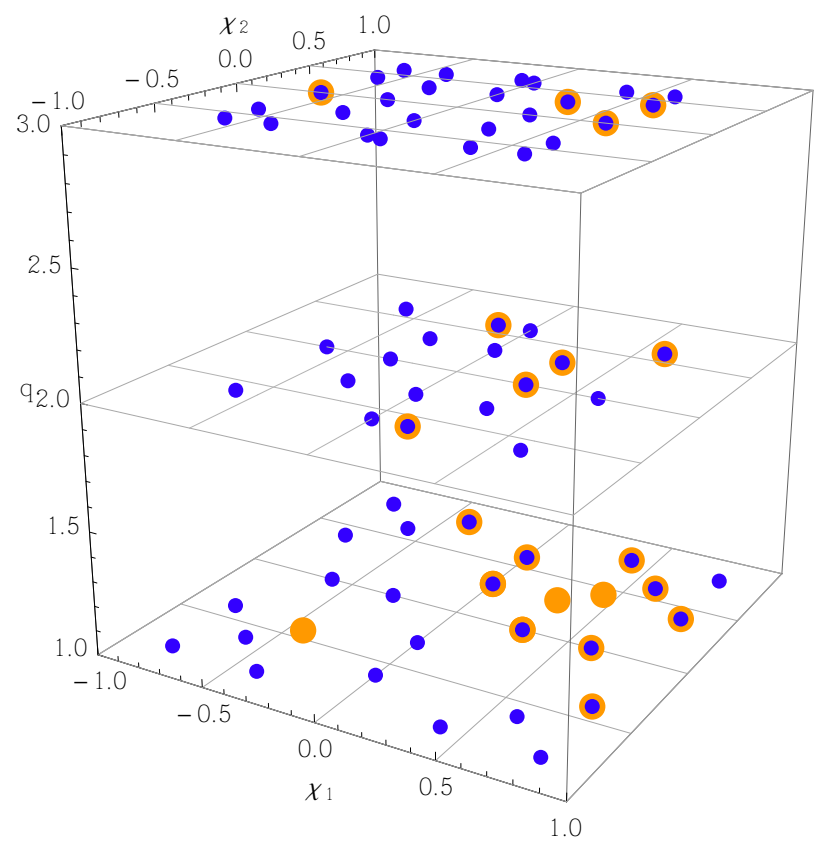

Figure 3. We show in the $\chi_{1}-\chi_{2}-q$ parameter space the 15 spinning nonprecessing configurations simulated in this paper plus the two equal-mass $\chi_{1}=$ $\chi_{2}= \pm 0.44$ contributed simulations (big orange dots), as well as the remaining 43 simulations that were planned in the first stage of the NRAR collaboration (small blue dots). More details on the binary parameters of the 17 simulations that have been performed can be found in table 1 Note that in the $q=1$ plane, the points are symmetric in $\chi_{1}$ and $\chi_{2}$.

Except for a few equal-mass binary simulations with $\chi_{1}=0.8$, the binaries simulated in the first stage have mass ratio $1 \leq q \leq 3$, component spin magnitudes $\chi_{1,2} \leq$ 0.6 and initial angles between the spins and the orbital angular momentum of $0^{\circ}, 60^{\circ}, 120^{\circ}, 180^{\circ}$. $\dagger$ In figure 3 we show the 58 non-precessing configurations in the $\chi_{1^{-}} \chi_{2^{-}} q$ parameter space. They cover the parameter space quite evenly. In this paper we present 21 simulations from the first stage (16 non-precessing and 5 precessing) and 1 from the third stage. Moreover, 42 non-precessing simulations from the first stage are under production by the SXS collaboration. In table 1 we list the binary parameters of the 22 simulations produced for the NRAR project plus three nonprecessing contributed simulations. The initial data parameters used for all of these simulations are summarized in Appendix A Cases 12 and 25 were evolved using the same initial data in order to provide a direct comparison between waveforms produced using different codes. As we discuss below, those simulations can be used to test and improve current analytical non-precessing models $86,88,91,92$, and can be employed

††Note that we obtain the direction of the orbital angular momentum numerically using the Newtonian expression applied to the coordinate positions and momenta of the black holes. This is clearly gauge-dependent, but is the standard procedure, and we expect it to give reasonable results when the holes are (relatively) far apart, as they are near the beginning of the simulation, where we calculate this quantity. 
Table 1. Configurations included in this study. The waveforms are numbered in the first column, while the second and third columns give the simulation group and a descriptive label. The label is composed of the initial of the simulation team (see table 2, the mass-ratio $q$, the letter 'p' in the case of precessing binaries, and the components of the initial dimensionless spins along the orbital angular momentum multiplied by 100 (e.g. the ' +15 ' in the label for Case $6(\mathrm{G} 2+15-60)$ corresponds to $\hat{\mathbf{L}} \cdot \mathbf{S}_{1} / m_{1}^{2}=+0.15$, where $\hat{\mathbf{L}}$ denotes the direction of the orbital angular momentum). We have marked the three contributed waveforms with asterisks. $q$ is the mass ratio $m_{1} / m_{2}$ where $m_{i}$ is the mass at $t_{0} . \mathbf{S}_{i} / m_{i}^{2}$ indicates the components of the dimensionless spin at $t_{0}$ in an orthonormal, right-handed frame where the black holes are on the $x$-axis and the orbital angular momentum points along the $z$-axis. For the non-precessing cases, where the spins are aligned with the orbital angular momentum, we only give the $z$-component of the spins, since the other components are zero. In Cases 1-4 and 23, the spins at $t_{0}$ were not output, so we give the initial spins. $M_{f}$ is the mass of the final black hole, and $M=m_{1}+m_{2} .\left|\chi_{f}\right|$ is the norm of the dimensionless spin of the final black hole.

\begin{tabular}{|c|c|c|c|c|c|c|c|}
\hline$\#$ & Group & Label & $q$ & $\mathbf{S}_{1} / m_{1}^{2}$ & $\mathbf{S}_{2} / m_{2}^{2}$ & $M_{f} / M$ & $\left|\chi_{f}\right|$ \\
\hline 1 & JCP & $\mathrm{J} 1 \mathrm{p}+49+11$ & 1 & $(-0.128,0.171,0.494)$ & $(0.129,-0.149,0.106)$ & 0.941 & 0.774 \\
\hline 2 & & $\mathrm{~J} 2-15+60$ & 2 & -0.150 & 0.600 & 0.961 & 0.611 \\
\hline 3 & FAU & $\mathrm{F} 1 \mathrm{p}+30-30$ & 1 & $(0.000,-0.520,0.300)$ & $(0.520,0.000,-0.300)$ & 0.952 & 0.704 \\
\hline 4 & & $\mathrm{~F} 3+60+40$ & 3 & 0.600 & 0.400 & 0.958 & 0.800 \\
\hline 5 & GATech & $\mathrm{G} 1+60+60$ & 1 & 0.603 & 0.603 & 0.927 & 0.858 \\
\hline 6 & & $\mathrm{G} 2+15-60$ & 2 & 0.150 & -0.607 & 0.962 & 0.635 \\
\hline 7 & & $\mathrm{G} 2+30+00$ & 2 & 0.301 & 0.000 & 0.955 & 0.717 \\
\hline 8 & & $\mathrm{G} 2+60+60$ & 2 & 0.601 & 0.607 & 0.940 & 0.839 \\
\hline 9 & RIT & R10 & 10 & 0.000 & 0.000 & 0.992 & 0.263 \\
\hline 10 & Lean & $\mathrm{L} 4 *$ & 4 & 0.000 & 0.000 & 0.978 & 0.472 \\
\hline 11 & & $\mathrm{~L} 3+60+00$ & 3 & 0.600 & 0.000 & 0.957 & 0.792 \\
\hline 12 & AEI & $\mathrm{A} 1+30+00$ & 1 & 0.300 & 0.000 & 0.947 & 0.732 \\
\hline 13 & & $\mathrm{~A} 1+60+00$ & 1 & 0.602 & 0.000 & 0.942 & 0.775 \\
\hline 14 & $\mathrm{PC}$ & $\mathrm{P} 1+80-40$ & 1 & 0.802 & -0.400 & 0.945 & 0.744 \\
\hline 15 & & $\mathrm{P} 1+80+40$ & 1 & 0.801 & 0.400 & 0.927 & 0.856 \\
\hline 16 & SXS & $\mathrm{S} 1+44+44^{*}$ & 1 & 0.437 & 0.437 & 0.936 & 0.814 \\
\hline 17 & & $\mathrm{~S} 1-44-44^{*}$ & 1 & -0.438 & -0.438 & 0.961 & 0.548 \\
\hline 18 & & $\mathrm{~S} 1+30+30$ & 1 & 0.300 & 0.300 & 0.942 & 0.775 \\
\hline 19 & & $\mathrm{~S} 2+30+30$ & 2 & 0.300 & 0.300 & 0.953 & 0.734 \\
\hline 20 & & $\mathrm{~S} 3+30+30$ & 3 & 0.300 & 0.300 & 0.965 & 0.680 \\
\hline 21 & & $\mathrm{~S} 3 \mathrm{p}+00-15$ & 3 & 0.000 & $(0.260,0.005,-0.150)$ & 0.972 & 0.536 \\
\hline 22 & & $\mathrm{~S} 1 \mathrm{p}+30+30$ & 1 & $(0.054,-0.514,0.305)$ & $(0.054,-0.514,0.305)$ & 0.937 & 0.804 \\
\hline 23 & & $\mathrm{~S} 1 \mathrm{p}-30-30$ & 1 & $(0.000,0.520,-0.300)$ & $(0.000,0.520,-0.300)$ & 0.958 & 0.638 \\
\hline 24 & & $\mathrm{~S} 3-60+00$ & 3 & -0.599 & 0.000 & 0.978 & 0.271 \\
\hline 25 & UIUC & $\mathrm{U} 1+30+00$ & 1 & 0.300 & 0.000 & 0.947 & 0.732 \\
\hline
\end{tabular}

to start building precessing models.

The second stage will consist of a larger number of precessing binary simulations, still with mild mass ratios and spin magnitudes. These will cover the precessing parameter space more densely and allow the construction of analytical precessing waveforms to be used by the advanced LIGO and Virgo detectors to detect precessing systems. Finally, the third stage will be devoted to several challenging simulations which either have high mass ratios $(3 \leq q \leq 15)$, large spin magnitudes $\left(0.6<\chi_{1,2}<\right.$ 1 ), or many orbits (say, $\sim 50$ orbits before merger). It is quite important to test the performance of analytical waveforms against numerical-relativity waveforms that are much longer than the ones used to calibrate the analytical waveforms. We note that the $q=10$ non-spinning simulation completed in this paper (see table 1) already 
Table 2. Abbreviations for NR group names, as used in table 1 and throughout the paper.

\begin{tabular}{ll}
\hline Abbreviation & Group name \\
\hline JCP & Jena-Cardiff-Palma \\
FAU & Florida Atlantic University \\
GATech & Georgia Tech \\
RIT & Rochester Institute of Technology \\
Lean & Ulrich Sperhake \\
AEI & Albert Einstein Institute \\
PC & Palma-Caltech \\
SXS & Simulating eXtreme Spacetimes \\
& (Caltech, Cornell, CITA, CSU Fullerton) \\
UIUC & University of Illinois, Urbana-Champaign \\
\hline
\end{tabular}

belongs to the third stage of the NRAR project. Simulations in the third stage can also be used to test the limits of analytical models developed in the first two stages and to guide our choices for future simulations.

To facilitate rapid progress toward the goal of building accurate waveforms to be used for detection by the advanced LIGO and Virgo detectors, the NSF made available to the NRAR collaboration an allocation of 11 million CPU hours on the Teragrid (now XSEDE) machine Kraken. This allocation, together with the computer resources of individual numerical-relativity groups, was used to carry out the 22 simulations presented in this paper. In addition to the waveforms produced specifically for this project, three previously-produced waveforms (Cases $10(\mathrm{~L} 4), 16(\mathrm{~S} 1+44+44)$ and 17 (S1-44-44) in table 1) were included in the analysis.

Before concluding this section on the selection of numerical simulations, we notice that since the inception of the NRAR collaboration in 2010, two algorithms have been proposed to reduce the dimensionality of the template bank: the singular-valuedecomposition technique [116 118] and the reduced-basis formalism [119 121]. These algorithms could be employed in the future to span the binary parameter space more efficiently.

\subsection{Accuracy and length requirements}

Longer and/or more accurate waveforms are more costly to produce than shorter and/or less accurate waveforms, both in terms of computational cost and in terms of human effort. Therefore, a trade-off is necessary between length and accuracy on the one hand, and breath of parameter-space coverage (number of performed simulations) on the other hand. A series of recent studies has addressed the length and accuracy requirements of numerical-relativity waveforms that are to be used for gravitationalwave data analysis.

A strict upper bound on the length and accuracy of waveforms can be obtained if one requires that the effect of all errors that enter the construction of gravitational waveforms do not lead to any observable consequences in gravitationalwave detectors 122 126]. This point of view was examined in $35,89,92,127,131$, These studies showed that the error budget was dominated by the PN waveforms that

$\ddagger \ddagger$ However, note that these studies did not take the detector's calibration error into account, which can increase the waveform accuracy requirements, as discussed in 124. 
are needed to represent the waveforms before the start of the numerically-computed late inspiral and merger. In most cases the impact of the PN errors decreases with increasing order of the PN expansion. In the non-spinning case, at the presently available $3.5 \mathrm{PN}$ order, numerical relativity may have to perform simulations lasting a hundred or several hundreds of orbits (this number generally increases for larger mass ratios) in order to completely control the errors due to the PN expansion. Hundreds of numerical simulations of this length are impractical today.

It is worth pointing out that the criterion suggested in 125,126 is a sufficient but not necessary requirement for avoiding observable consequences, and it does not say which of the binary parameters will be biased and how large the bias will be. Using the criterion suggested in 125, 126, the authors of 86] concluded that the analytical template family developed in $[86]$ would lead to systematic errors larger than the statistical errors for SNR $=10$ when $q \gtrsim 6$ and the total mass is $>100 M_{\odot}$. However, a direct study 132 carried out with the Markov Chain Monte Carlo technique demonstrated that the template family in 86 is indistinguishable from the numericalrelativity waveforms 66 used to calibrate it up to $\mathrm{SNR}=50$ for the advanced LIGO detectors.

Furthermore, the very first task of gravitational-wave observatories is the detection of signals. Gravitational-wave searches merely require that one of the search templates matches the exact waveform, rather than the template with the same mass and spin parameters as the exact waveform. This criterion of 'effectualness' is much weaker, and 128,130 ] find that approximately 10 numerical-relativity orbits are sufficient for aligned spin binary black holes with moderate spins and moderate mass ratios; for non-spinning binaries 10 orbits are sufficient up to $q \sim 20$. This study also finds that in these cases parameter biases are not likely to affect the astrophysical information that can be inferred from observations. Indeed, the parameter uncertainties due to degeneracies between waveform parameters will in many cases be the dominant source of error for advanced-detector observations 133 135].

Unfortunately, none of these earlier studies is fully applicable to our task. We would like to cover precessing systems, for which accuracy requirements have not yet been studied. Furthermore, instead of simply attaching an existing PN approximant to the numerical waveforms, we intend to calibrate analytical models to the numericalrelativity waveforms. Presumably, a calibration with free parameters (e.g. fourth order PN coefficients) will represent the true waveform better than just a given PN waveform. Unfortunately, it is currently not known how much better, because no longer numerical waveforms exist to compare against (although such longer waveforms are becoming available, see e.g. 35,51]). Earlier studies that calibrated EOB models to numericalrelativity simulations $[31,82,88$ succeeded in pushing the calibration errors to within the numerical truncation error over the entire length of the numerical-relativity simulation. Thus, there is certainly benefit in having waveforms of comparable length (inspiral of $\sim 30$ gravitational-wave cycles) and comparable accuracy (phase error of $\sim 0.05$ radians during the inspiral) to these simulations already used for calibration. However, these criteria are very challenging for numerical-relativity codes - for instance, the NINJA-2 collaboration [99, had a target of 10 usable gravitational-wave cycles and a gravitational-wave phase accuracy of 0.5 radians.

The discussion above shows that there are clear benefits from having higher quality waveforms, where 'higher quality' refers to longer inspirals and smaller numerical errors. However, attempting to increase waveform quality too much over the status quo will be very expensive, and may be hindered by new issues in the 
numerical-relativity codes which may appear when the codes are pushed to compute waveforms of unprecedented length and accuracy. Therefore, we only modestly tighten the tolerances on the quality of the numerical-relativity waveforms, sharpening them by about a factor of two relative to what was achieved in the NINJA-2 project, noting that this corresponds to an increase of significantly more than a factor of two in computational cost.

Specifically, we target:

- About 20 usable gravitational-wave cycles between $t_{0}$ and $t_{\text {ref }}$, where $t_{0}$ is the time after which the effects of 'junk-radiation' - due to the use of non-astrophysical initial data - are no longer visible in the waveform, and $t_{\text {ref }}$ is the time at which the gravitational-wave frequency of the $(2,2)$ mode $M \omega_{\mathrm{GW}}=M \omega_{22}$, ref $\equiv 0.2$.

- A relative amplitude error of the $(2,2)$ mode of the gravitational waves of $\delta A_{22} / A_{22} \lesssim 0.01$ up to the gravitational-wave frequency $M \omega_{22}$, ref $=0.2$.

- A cumulative phase error of $\lesssim 0.25$ radians up to the gravitational-wave frequency $M \omega_{22, \text { ref }}=0.2$.

- Orbital eccentricity $e \lesssim 0.002$.

These criteria form guidelines for the present work. We relax some of them for particularly challenging simulations like the mass ratio $q=10$ case. While in general, most groups have stayed close to the guidelines to maximize parameter-space coverage, we nevertheless have several longer waveforms in the catalog which we will use to gain further insight into ongoing research into length requirements for numerical waveforms.

\section{Numerical-relativity codes}

For the numerical solution of the Einstein field equations, it is necessary to recast the equations in the form of an initial value problem, where one starts from an initial snapshot of the physical system under consideration and evolves forward in time. Approaches to achieve this goal can be classified into (i) characteristic schemes effectively based on the characteristics or light cones of the equations and (ii) Cauchy or ' $3+1$ ' splits where spacetime is decomposed into a one-parameter family of spatial hypersurfaces. Simulations of black-hole binary systems have so far only been performed with Cauchy methods and we shall focus our discussion on these $3+1$ methods. For more details on the characteristic approach see [136].

Quite remarkably, after nearly forty years of research, the breakthrough in numerically evolving black-hole binaries through inspiral and merger was achieved within a relatively short period of time using two significantly different $3+1$ frameworks: Pretorius' 37 work employing the generalized harmonic gauge (GHG) formulation 137 139 combined with black-hole excision, and the moving punctures technique developed by the Brownsville and Goddard groups [38, 39 based on the Baumgarte-Shapiro-Shibata-Nakamura (BSSN) formulation 140.141. (See 142 144 for a theoretical discussion of the moving puncture approach in the Schwarzschild spacetime.) These methods provided the community with two independent approaches to the simulation of black-hole mergers, and the opportunity to validate both via a comparison of the resulting gravitational waveforms [100]. We will briefly review the methods here and point interested readers to the references listed for the individual codes in table 3 . 


\subsection{The Spectral Einstein Code}

The Spectral Einstein Code SpEC [145] used by the SXS collaboration is a pseudospectral multi-domain code that implements a first-order representation 146 of the generalized harmonic system $137-139$. The evolution variables are the ten components of the lower index spacetime metric $\psi_{a b}$ along with the auxiliary variables $\Pi_{a b}$ and $\Phi_{i a b}$ introduced in the process of converting the original (second differential order) system into a first-order representation. Latin letters from the beginning of the alphabet represent space-time indices $(a, b, c, d=0,1,2,3)$, whereas latin letters from the middle of the alphabet represent spatial indices $(i, j, \ldots=1,2,3)$. The equations are given by

$$
\begin{aligned}
\partial_{t} \psi_{a b}- & \left(1+\gamma_{1}\right) \beta^{k} \partial_{k} \psi_{a b}=-\alpha \Pi_{a b}-\gamma_{1} \beta^{i} \Phi_{i a b} \\
\partial_{t} \Pi_{a b}- & \beta^{k} \partial_{k} \Pi_{a b}+\alpha g^{k i} \partial_{k} \Phi_{i a b}-\gamma_{1} \gamma_{2} \beta^{k} \partial_{k} \psi_{a b} \\
= & 2 \alpha \psi^{c d}\left(g^{i j} \Phi_{i c a} \Phi_{j d b}-\Pi_{c a} \Pi_{d b}-\psi^{e f} \Gamma_{a c e} \Gamma_{b d f}\right)-2 \alpha \nabla_{(a} H_{b)} \\
& -\frac{1}{2} \alpha t^{c} t^{d} \Pi_{c d} \Pi_{a b}-\alpha t^{c} \Pi_{c i} g^{i j} \Phi_{j a b} \\
& +\alpha \gamma_{0}\left[2 \delta^{c}{ }_{(a} t_{b)}-\psi_{a b} t^{c}\right]\left(H_{c}+\Gamma_{c}\right)-\gamma_{1} \gamma_{2} \beta^{i} \Phi_{i a b} \\
\partial_{t} \Phi_{i a b}- & \beta^{k} \partial_{k} \Phi_{i a b}+\alpha \partial_{i} \Pi_{a b}-\alpha \gamma_{2} \partial_{i} \psi_{a b} \\
= & \frac{1}{2} \alpha t^{c} t^{d} \Phi_{i c d} \Pi_{a b}+\alpha g^{j k} t^{c} \Phi_{i j c} \Phi_{k a b}-\alpha \gamma_{2} \Phi_{i a b} .
\end{aligned}
$$

In (2)-(4) we used $\alpha, \beta^{i}$ for the $3+1$ lapse and shift and $g^{i j}$ for the inverse of the spatial metric (which differs from the spatial components of the inverse space-time metric $\left.\psi^{a b}\right)$. Furthermore, the space-time vector $t^{a}$ represents the future directed time-like unit normal to the constant- $t$ hypersurfaces, and $\gamma_{0}, \gamma_{1}, \gamma_{2}$ are constraint damping parameters. We have also made use of the four-dimensional Kronecker-delta, $\delta^{a}{ }_{b}$, the four-dimensional Christoffel symbols, $\Gamma_{a b c}$, and of their trace, $\Gamma_{a}=\psi^{b c} \Gamma_{a b c}$. (See 146 for details.)

In this formulation the gauge source functions $H_{a}=\psi_{a b} \nabla_{c} \nabla^{c} x^{b}$ are freelyspecifiable expressions depending on the coordinates $x^{a}$ and the metric components but not on the metric derivatives. At the beginning of our simulations we set these so as to minimize the dynamics of the lapse and shift. For low-spin systems (dimensionless spin $\leq 0.5)$ they are transitioned smoothly in time to harmonic gauge $\left(H_{a}=0\right)$ during the inspiral, while near merger we use the damped harmonic gauge condition

$$
H_{a}=\mu_{0}\left[\ln \left(\frac{\sqrt{g}}{\alpha}\right)\right]^{2}\left[\ln \left(\frac{\sqrt{g}}{\alpha}\right) t_{a}-\alpha^{-1} g_{a i} \beta^{i}\right],
$$

where $\mu_{0}$ is a free coefficient, $g$ is the determinant of the 3-metric and $g_{a i}$ is the spatial metric of the constant $-t$ hypersurfaces. (See 147, 148 for details.) For highspin systems (dimensionless spin $>0.5$ ) we transition to the damped harmonic gauge from the beginning of the simulation.

The SpEC simulations presented here utilize a large number of recent improvements, many of which were driven by the NRAR project itself. Two types of initial data are used: conformally flat quasi-equilibrium initial data 149,150 for lowspin systems and superposed Kerr-Schild initial data 56 for higher spins. Initial-data parameters are tuned to achieve desired physical masses and spins with the root-finding procedure described in [66]. Eccentricity removal for precessing binaries is described in [151. Orbital-plane precession is accounted for by parameterizing the rotation between grid frame and inertial frame using quaternions 152; this technique works as well for non-precessing binaries as do earlier approaches 29,103, 153, and is therefore 
used for all cases. Simulations of the inspiral phase of conformally flat initial data use the domain decomposition described in 29 based on constraint-damping parameters found in 154. Simulations of the inspiral phase of superposed Kerr-Schild initial data use a domain decomposition of touching domains described in 66. Mergers and ringdowns of all inspiral simulations, as well as the inspirals of superposed KerrSchild initial data, are performed with the coordinate mappings and control systems described in 155]. The location of the outer boundary is chosen as a multiple of the initial separation of the black holes and is in the range 450 to $650 \mathrm{M}$.

Time stepping in SpEC is performed with an eighth-order Dormand-Prince time stepper [156], with adaptive time stepping based on a fifth-order embedded updating formula. Output at evolution times other than the precise end of a time step utilizes the embedded interpolation formula of the Dormand-Prince time stepper.

SpEC's apparent horizon finder expands the radius of the apparent horizon as a series in spherical harmonics up to some order $L$. We utilize the fast flow methods developed by Gundlach 157 to determine the expansion coefficients. The quasi-local spin $S$ of each black hole is computed with the spin diagnostics described in 56, based on an angular momentum surface integral [158, 159] using approximate Killing vectors [56, 160 of the apparent horizons.

Gravitational waves are extracted by constructing the Newman-Penrose scalar $\Psi_{4}$ on a set of coordinate spheres far from the source, and decomposing into spinweighted spherical harmonics of weight -2 . Multiple extraction spheres are used to enable extrapolation of the waveform to infinite radius. The $\Psi_{4}$ extraction method used by SpEC is described in more detail in Refs. 29, 103,161 .

\subsection{Moving Punctures Codes}

The moving punctures method [38 39] is based on a canonical ' $3+1$ ' or Arnowitt-DeserMisner (ADM) 162 split of the Einstein field equations reformulated by York 163] which recasts the equations in terms of the spatial metric $\gamma_{i j}$ and the extrinsic curvature $K_{i j}$ as well as a lapse function $\alpha$ and shift vector $\beta^{i}$, which represent the coordinate or gauge freedom of general relativity. In this form, the field equations appear as a set of six evolution equations each for $\gamma_{i j}$ and $K_{i j}$, and four constraint equations (the Hamiltonian and momentum constraints).

The ADM evolution equations are not strongly hyperbolic, and hence do not result in a well-posed initial value problem. They therefore cannot lead to a stable numerical discretisation using standard methods. However, by mixing the constraint equations into the evolution system in specific ways, the evolution system can be made strongly hyperbolic. The BSSN system is one such reformulation, and has been shown $164-166$ to be strongly hyperbolic $\S \S$. In addition to the modification of the evolution system using the constraint equations, the BSSN system also includes a decomposition of the extrinsic curvature into trace and trace-free parts, a conformal transformation and the introduction of the contracted Christoffel symbols as independent variables. These modifications make the system suitable for moving-punctures evolutions.

$\S \S$ Technically, for the gauge choices commonly used, this is true everywhere in the domain except on sets of measure zero, but the effects of the failure of strong hyperbolicity there are negligible. 
The evolution variables used in the BSSN system are defined as

$$
\begin{aligned}
& \phi=\frac{1}{12} \ln \gamma, \quad \tilde{\gamma}_{i j}=e^{-4 \phi} \gamma_{i j}, \\
& K=\gamma^{i j} K_{i j}, \quad \tilde{A}_{i j}=e^{-4 \phi}\left(K_{i j}-\frac{1}{3} \gamma_{i j} K\right), \\
& \tilde{\Gamma}^{i}=\tilde{\gamma}^{m n} \tilde{\Gamma}_{m n}^{i},
\end{aligned}
$$

where $\gamma$ denotes the determinant of $\gamma_{i j}$ and $\tilde{\Gamma}_{m n}^{i}$ are the Christoffel symbols associated with the conformal spatial metric $\tilde{\gamma}_{i j}$. The evolution equations are given by

$$
\begin{aligned}
\partial_{t} \tilde{\gamma}_{i j}= & \beta^{m} \partial_{m} \tilde{\gamma}_{i j}+2 \tilde{\gamma}_{m(i} \partial_{j)} \beta^{m}-\frac{2}{3} \tilde{\gamma}_{i j} \partial_{m} \beta^{m}-2 \alpha \tilde{A}_{i j} \\
\partial_{t} \phi= & \beta^{m} \partial_{m} \phi+\frac{1}{6}\left(\partial_{m} \beta^{m}-\alpha K\right) \\
\partial_{t} \tilde{A}_{i j}= & \beta^{m} \partial_{m} \tilde{A}_{i j}+2 \tilde{A}_{m(i} \partial_{j)} \beta^{m}-\frac{2}{3} \tilde{A}_{i j} \partial_{m} \beta^{m} \\
& +e^{-4 \phi}\left(\alpha R_{i j}-D_{i} D_{j} \alpha\right)^{\mathrm{TF}}+\alpha\left(K \tilde{A}_{i j}-2 \tilde{A}_{i}^{m} \tilde{A}_{m j}\right) \\
\partial_{t} K= & \beta^{m} \partial_{m} K-D^{m} D_{m} \alpha+\alpha\left(\tilde{A}^{m n} \tilde{A}_{m n}+\frac{1}{3} K^{2}\right) \\
\partial_{t} \tilde{\Gamma}^{i}= & \beta^{m} \partial_{m} \tilde{\Gamma}^{i}-\tilde{\Gamma}^{m} \partial_{m} \beta^{i}+\frac{2}{3} \tilde{\Gamma}^{i} \partial_{m} \beta^{m}+2 \alpha \tilde{\Gamma}_{m n}^{i} \tilde{A}^{m n}+\frac{1}{3} \tilde{\gamma}^{i m} \partial_{m} \partial_{n} \beta^{n} \\
& +\tilde{\gamma}^{m n} \partial_{m} \partial_{n} \beta^{i}-\frac{4}{3} \alpha \tilde{\gamma}^{i m} \partial_{m} K+2 \tilde{A}^{i m}\left(6 \alpha \partial_{m} \phi-\partial_{m} \alpha\right)
\end{aligned}
$$

see for example Sec. II in Alcubierre et al. [167]. Here, $D_{i}$ and $R_{i j}$ are the covariant derivative and the Ricci tensor associated with the physical spatial metric $\gamma_{i j}$ and the superscript ' $\mathrm{TF}$ ' denotes the tracefree part.

When introducing a new variable (here $\tilde{\Gamma}^{i}$ ), it is necessary to choose in which places the new variable will be used, and in which the original will be used. Usually, the new variable is used wherever it appears. Either of the following two recipes yield a strongly hyperbolic system:

- Alcubierre et al. 167 use the variable $\tilde{\Gamma}^{i}$ wherever it appears differentiated, but use the original variable $\tilde{\gamma}^{m n} \tilde{\Gamma}_{m n}^{i}$ wherever it appears undifferentiated, i.e. in the computation of $R_{i j}$ and in the second and third terms of the right hand side of (11).

- Yo et al. [168 use $\tilde{\Gamma}^{i}$ everywhere, but add to the right-hand side of 11] a term

$$
\mathcal{C}^{i}=-\left(\sigma+\frac{2}{3}\right)\left(\tilde{\Gamma}^{i}-\tilde{\gamma}^{m n} \tilde{\Gamma}_{m n}^{i}\right) \partial_{k} \beta^{k}
$$

Here, $\sigma$ is a constant set to $\sigma=2 / 3$ for the simulations performed by the GATech group and $\sigma=0$ for those performed by the Lean group; cf. table 3

Note that the definition of the BSSN variables in (6) implies the auxiliary constraints

$$
\begin{aligned}
\operatorname{det} \tilde{\gamma}_{i j} & =1, \\
\operatorname{tr} \tilde{A}_{i j} & =0 .
\end{aligned}
$$

The continuum evolution system in which these constraints are not enforced is only weakly hyperbolic [169], leading to instability of the finite difference scheme. Strong 
hyperbolicity results if both constraints are enforced. Empirically (though this has not yet been formally proven), it is sufficient to explicitly enforce (14) after each time step in order to achieve numerical stability. This is accomplished in all codes by subtracting any residual trace contribution from $\tilde{A}_{i j}$ after each time step. In contrast, enforcing $\operatorname{det} \tilde{\gamma}_{i j}=1$ appears to be optional and is implemented only in some codes; cf. table 3 .

A further freedom exists in the choice of variable for evolving the conformal factor. Alternatives to the variable $\phi$ defined in (6) which have been suggested in the literature are $\chi=e^{-4 \phi} 38$ and $W=e^{-2 \phi}[170$.

In the moving punctures approach, the BSSN equations (7)-111) are complemented by the ' $1+\log$ ' slicing and a $\Gamma$-driver condition for the shift vector. These are given by

$$
\begin{aligned}
& \partial_{t} \alpha=\beta^{m} \partial_{m} \alpha-2 \alpha K, \\
& \partial_{t} \beta^{i}=\zeta_{\beta} \beta^{m} \partial_{m} \beta^{i}+\frac{3}{4} B^{i}, \\
& \partial_{t} B^{i}=\zeta_{\beta} \beta^{m} \partial_{m} B^{i}+\partial_{t} \tilde{\Gamma}^{i}-\zeta_{\beta} \beta^{m} \partial_{m} \tilde{\Gamma}^{i}-\eta B^{i} .
\end{aligned}
$$

Here, the auxiliary variable $B^{i}$ is defined through $(16), \zeta_{\beta}$ is a constant set to 0 or 1 that determines the inclusion of advection terms and $\eta$ is a free parameter or function of dimension length ${ }^{-1}$. The choices of $\zeta_{\beta}$ which yield a strongly hyperbolic system were determined in 165. Van Meter et al. 171] suggest an alternative first-orderin-time evolution equation for the shift vector obtained from integration of $(16)$ and (17), viz.

$$
\partial_{t} \beta^{i}=\zeta_{\beta} \beta^{m} \partial_{m} \beta^{i}+\frac{3}{4} \tilde{\Gamma}^{i}-\eta \beta^{i} .
$$

The evolution system (7)-11) is initialized using binary black hole Bowen-York data 172, 173 using a spectral solver 187 for the calculation of the conformal factor. Particular care is required for the choice of the Bowen-York parameter for the individual holes' linear momentum in order to obtain an initial black-hole binary configuration with (nearly) vanishing eccentricity. This is achieved in the individual codes either by employing post-Newtonian or EOB model predictions for the momenta 34, 176, 177, 188 or using iterative procedures as described in Refs. 151, 161, 176, 181, 189. The shift is initialized as $\beta^{i}=0$ whereas the initial lapse is given as some function of the conformal factor $\phi$. Additionally, the bare mass parameters in the Bowen-York initial data are determined by iterative methods to achieve the desired physical masses, often approximated by the ADM mass evaluated at the punctures 173,190$]$.

All moving punctures codes employ mesh refinement provided by CARPET 191] or BAM [174 192 193 and use 5th order Lagrange polynomial interpolation in space and 2nd order in time (6th order and 3rd order accurate respectively). The GATech, RIT, Lean, AEI, PC and UIUC groups use codes based on the CACTUS framework 194 195 and the Einstein Toolkit 196, 197]. The evolution equations are evolved using finite differencing in space combined with the method of lines with a fourth-order RungeKutta scheme for time integration. Kreiss-Oliger dissipation [198 is added to the evolution equations, characterized by the order $n_{\mathrm{KO}}$ which denotes the power of the spatial grid spacing $\Delta x$ appearing in the dissipation term; see e.g. 175, 180 for details. In addition to mesh refinement, the AEI and PC groups also employ the LlAMA multipatch infrastructure which discretizes the wavezone by a set of six overlapping spherical 'inflated cube' grid patches [184. In most codes, gravitational 
Table 3. Specifications of the moving-punctures codes. We list the choice of the variable $f_{\text {conf }}$ for the conformal factor, the choice for stably evolving $\tilde{\Gamma}^{i}$, the enforcement of the auxiliary constraint $\operatorname{det} \tilde{\gamma}_{i j}=1$, the evolution equations for the shift $\beta^{i}$, the gauge parameters $\eta$ and $\zeta_{\beta}$, the discretization orders $n_{\text {space }}$ in space and $n_{\mathrm{KO}}$ for the Kreiss-Oliger dissipation, the Courant factor $\Delta t / \Delta x$, the initialization of the lapse $\alpha(t=0)\left(\psi_{\mathrm{BL}}\right.$ is the Brill-Lindquist conformal factor given in e.g. (5) in 174), references to the methods employed for reduction of eccentricity in the initial data, the range of the location of the outer boundary of the computational domain $x_{\text {out }}$, and references containing more detailed descriptions of the various numerical codes. Entries of 'text' refer to a more detailed explanation given in Sec. 4.2

\begin{tabular}{|c|c|c|c|c|c|c|c|c|}
\hline Code & $f_{\text {conf }}$ & $\partial_{t} \tilde{\Gamma}^{i}$ & $\operatorname{det} \tilde{\gamma}_{i j}=1$ & $\partial_{t} \beta^{i}$ & $M \eta$ & $\zeta_{\beta}$ & $n_{\text {space }}$ & $n_{\mathrm{KO}}$ \\
\hline JCP & $\chi$ & 167 & yes & 16,17 & 2.0 & 1 & 6 & 5 \\
\hline FAU & $\chi$ & 167 & yes & 16,17 & 2.0 & 1 & 6 & 5 \\
\hline GATech & $\chi$ & 168 & no & $(\overline{16}),(\overline{17})$ & 2.0 & 1 & 6 & 7 \\
\hline RIT & $W$ & 167 & yes & 18 & text & 0 & 8 & 5 \\
\hline Lean & $\chi$ & 168 & no & 18 & text & 1 & 6 & 5 \\
\hline AEI & $W$ & 167 & no & 16,17 & 1.375 & 1 & 8 & 9 \\
\hline $\mathrm{PC}$ & $W$ & 167 & no & $(\overline{16}, \overline{17}$ & 1.0 & 1 & 8 & 9 \\
\hline UIUC & $\phi$ & 167. & yes & (18) & 1.375 & 0 & 6 & 5 \\
\hline
\end{tabular}

\begin{tabular}{|c|c|c|c|c|c|}
\hline Code & $\Delta t / \Delta x$ & $\alpha(t=0)$ & Ecc. & $x_{\text {out }} / M$ & Refs. \\
\hline JCP & 0.5 & $\psi_{\mathrm{BL}}^{-2}$ & text & $2050-3250$ & \begin{tabular}{|l|l|}
174 & 175 \\
\end{tabular} \\
\hline FAU & 0.25 & $\psi_{\mathrm{BL}}^{-2}$ & 176 & $774-1029$ & \begin{tabular}{|l|l|}
174 & 175 \\
\end{tabular} \\
\hline GATech & 0.5 & $\psi_{\mathrm{BL}}^{-2}$ & 177 & 410 & \begin{tabular}{|l|l|}
178 & 179 \\
\end{tabular} \\
\hline RIT & 0.25 & $2 /\left(1+1 / W^{2}\right)$ & 161 & 400 & $38 \quad 180$ \\
\hline Lean & 0.5 & $\sqrt{\chi}$ & 181 & $307-768$ & \begin{tabular}{|l|l|}
182 & 183 \\
\end{tabular} \\
\hline AEI & 0.45 & $\psi_{\mathrm{BL}}^{-1}$ & 177 & $3128-3400$ & 184 \\
\hline $\mathrm{PC}$ & 0.45 & $\psi_{\mathrm{BL}}^{-1}$ & 177 & 3400 & $\overline{184}$ \\
\hline UIUC & 0.45 & $\psi_{\mathrm{BL}}^{-1}$ & 177 & 384 & \begin{tabular}{|l|l|}
185 & 186 \\
\end{tabular} \\
\hline
\end{tabular}

waves are extracted by interpolating the Newman-Penrose scalar $\Psi_{4}$ onto spheres of constant coordinate radius $R_{\mathrm{ex}}$ and performing a decomposition into multipoles using spherical harmonics of spin weight $s=-2$ (see for example Sec. II in [199]). The PC group extracts gravitational waves directly at future null infinity $\mathcal{J}^{+}$using the method of Cauchy-characteristic extraction (CCE) 136 200. Information about the black-hole properties throughout the evolution is obtained from the apparent horizons 201 202] and spin estimates are obtained through approximate Killing vectors integrated on the horizon 203 or the relation between the horizon area and equatorial circumference 204.

The degrees of freedom of the individual simulations performed with the moving punctures technique can be summarized as follows.

- The choice of evolution variable for the conformal factor.

- The evolution of the variable $\tilde{\Gamma}^{i}$ using either the method suggested in 167 or that from 168.

- The enforcement of $\operatorname{det} \tilde{\gamma}_{i j}=1$.

- Evolution of the shift using the second-order equations (16), (17) or the first-order 
equation 18.

- The choice of $\eta$ and $\zeta_{\beta}$ in the shift condition.

- The order of spatial finite differencing of the evolution equations and the order of the Kreiss-Oliger dissipation.

- The Courant factor $\Delta t / \Delta x$, which needs to be sufficiently small to provide numerical stability.

- The initialization of the lapse function.

- The method employed for reducing eccentricity in the initial data.

- The placement of the outer boundary of the computational domain.

In table 3 we list the corresponding choices made in the individual moving punctures codes. For a few choices, individual codes use more elaborate implementations. The corresponding entries are labeled 'text' in the table and the descriptions of the methods are given as follows.

Courant factor: All codes use the Courant factor given in table 3 on the inner refinement levels. However, all but the Lean code decrease the Courant factor in the outer levels as follows. The RIT group decreases it on the four coarsest (i.e. outermost) levels by factors of $2,2,4$ and 8 in outgoing order relative to the base value. The JCP group decreases it by a factor of 2 consecutively going outwards on the 6 outermost levels. The FAU and GATech groups do the same on the four outermost levels, and the UIUC group on the three outermost levels. (For these four codes, this decrease of the Courant factor leads to a constant time step in the indicated levels.) The AEI and PC groups, however, decrease the Courant factor by a factor of 2 a single time on their outermost Cartesian patch and use the resulting value on the spherical patches extending to larger radii, as well.

$J C P$ : Low-eccentricity initial parameters were estimated for Case $1(\mathrm{~J} 1 \mathrm{p}+49+11)$ using the method from 34, 177 and for Case $2(\mathrm{~J} 2-15+60)$ using the method from 188].

RIT: For the choice of the shift parameter $\eta$, the RIT group uses a modification of the form proposed in 205. This modification detailed in 206 sets $\eta\left(x^{i}, t\right)=$ $R_{0} \sqrt{\tilde{\gamma}^{i j} \partial_{i} W \partial_{j} W}\left(1-W^{a}\right)^{-b}$ with the specific choice $R_{0}=1.31, a=b=2$. Once the conformal factor settles down to its asymptotic form of $\psi=C / \sqrt{r}+O(1)$ near the puncture, this definition implies that $\eta$ will have the form $\eta=\left(R_{0} / C^{2}\right)\left(1+b\left(r / C^{2}\right)^{a}\right)$ near the puncture and $\eta=R_{0} r^{b-2} M /(a M)^{b}$ as $r \rightarrow \infty$. This modification is designed to treat large mass ratio binaries.

Lean: The Lean simulations have used a position-dependent shift parameter $\eta$. For simulation L4, this function is given by $M \eta_{\mathrm{L} 4}=\eta_{0}\left(r_{1}+r_{2}\right) /\left[\left(1+q^{-1}\right)^{-1} r_{2}+(1+q)^{-1} r_{1}\right]$, where $r_{i}=\left|\mathbf{x}-\mathbf{x}_{i}\right|$ is the coordinate distance of the grid point from the location of the $i$ th black hole and $\eta_{0}=0.7$. For simulation $\mathrm{L} 3+60+00, M \eta_{\mathrm{L} 3}=\left[r_{0}^{2} /\left(r^{2}+r_{0}^{2}\right)\right] \times M \eta_{\mathrm{L} 4}$ where $\eta_{0}=1.0$ (instead of 0.7$), r$ is the distance of the grid point from the origin and $r_{0}$ a constant set to $192 \mathrm{M}$; cf. 207]. Note that with this notation $\eta_{0}$, unlike $\eta$, is dimensionless. 


\section{Numerical-relativity waveforms}

\subsection{Strain waveforms}

For the purpose of binary-black-hole gravitational-wave science, it is necessary to determine the metric strain $h$ very far from the source. In practice, the waveform at future null infinity, $\mathcal{J}^{+}$, is desired. This can be directly computed using the method of Cauchy-characteristic extraction (CCE) [136, 200, 208, or obtained by computing the waveform in the simulation at very large but finite radius, or by extrapolating several finite-radius measurements. Waveforms can be computed at finite radius using the Zerilli formalism 209 or from the Newman-Penrose scalar $\Psi_{4}$. A recent investigation of the detailed relation between the two methods can be found in [210]. The metric strain at $\mathcal{J}^{+}$must then be computed from these finite-radius measurements. While each group may use more than one method simultaneously to compute gravitational waves, we present results using $\Psi_{4}$ as this is the only method implemented by all groups.

The $\Psi_{4}$ waveforms from the Palma-Caltech group are computed directly at $\mathcal{J}^{+}$ using CCE, while the waveforms from all the other groups are computed at finite radii and extrapolated.

Computation of strain Waveform modes of $\Psi_{4}$ are typically computed at several radii as

$$
C_{\ell m}(t, r)=\int{ }^{-2} Y_{\ell m}^{*}(\theta, \phi) r \Psi_{4}(t, r, \theta, \phi) d \Omega,
$$

where ${ }^{-2} Y_{\ell m}$ are the spherical harmonics of spin weight $s=-2$ (see 199 for notation and conventions) and the star denotes the complex conjugate.

To compute $h$ from $\Psi_{4}$ at finite radius, we use the method of fixed-frequency integration (FFI) 211]. Unless the waveform is obtained via CCE, the waveform at $\mathcal{J}^{+}$is computed by extrapolating $h$ from several finite radii.

In the Bondi gaug $\Phi \mathbb{\Phi}$, the strain, $h$, and $\Psi_{4}$ are related by the simple relation $\Psi_{4}=\ddot{h}$. Performing two integrations in the time domain (even if the correct constants can be determined) can lead to unphysical artefacts that severely contaminate the waveform, in particular the sub-dominant modes. Specifically, small perturbations due to numerical noise are amplified unacceptably leading to long-term non-linear drifts in the amplitude; see 213 for examples. The FFI method involves performing the integration in the Fourier domain, the usual division by $\omega$ being replaced by a division by $\omega_{0}$ for $|\omega|<\omega_{0}$ to avoid the phenomenon of spectral leakage. The method is motivated and described in detail in [211].

The Discrete Fourier Transform (DFT) of the discretely sampled $* * * \Psi_{4}$ waveform mode, $C_{j}=C_{\ell m}\left(t_{j}, r\right), j=0,1, \ldots, N-1$, is given by

$$
\tilde{C}_{k}=\frac{1}{\sqrt{N}} \sum_{j=0}^{N-1} C_{j} \mathrm{e}^{2 \pi \mathrm{i} j k / N} .
$$

ฯ 9 See 212 for a discussion of the effects of waveform computation in gauges which are only approximately Bondi.

$* * *$ In the case where the waveform is computed on a nonuniformly-spaced grid $t_{i}$, the data is first interpolated onto a uniformly-spaced grid. 
(Note that the $\ell m$ mode labels are omitted for discretized quantities such as $C_{j}$ for brevity.) The strain, $H_{k}$, is computed by time integration as

$$
\tilde{H}_{k}=\frac{\tilde{C}_{k}}{\Omega^{2}(\omega)},
$$

where

$$
\Omega(\omega)=\left\{\begin{aligned}
\omega_{0} & \text { if }|\omega|<\omega_{0} \\
\omega & \text { if }|\omega| \geq \omega_{0}
\end{aligned}\right.
$$

The choice of $\omega_{0}$ is guided by the principle that it should be smaller than any important physical frequencies present in the problem, but not so small as to introduce significant spectral leakage 200. In practice, we have found acceptable errors when using

$$
\begin{aligned}
& \omega_{0}=m \frac{\omega_{I}}{4}, \\
& \omega_{I}=\left.\frac{d}{d t} \arg C_{22}\right|_{t=t_{0}},
\end{aligned}
$$

where $m$ is the spherical harmonic mode index and $\omega_{I}$ is the initial frequency of $C_{22}$ measured after the junk radiation at $t=t_{0}$. Once a value of $\omega_{0}$ has been determined for $m=2$, values for $\omega_{0}$ for the other modes can be obtained from 22.

We then compute the inverse DFT,

$$
H_{j}=\frac{1}{\sqrt{N}} \sum_{k=0}^{N-1} \tilde{H}_{k} \mathrm{e}^{-2 \pi \mathrm{i} k j / N}
$$

to obtain $H_{\ell m}(t, r)$.

Extrapolation of strain to $\mathcal{J}^{+}$The extrapolation of finite-radius waveforms is not the same as a direct computation at $\mathcal{J}^{+}$, and introduces a degree of uncertainty associated with the extrapolation error (see Sec. 5.2). The agreement between extrapolated results and those obtained at $\mathcal{J}^{+}$using CCE was investigated in 200.

Gravitational waves far from an isolated source propagate - to a good approximation - along outgoing radial null geodesics. In the Schwarzschild spacetime, these are given by

$$
u=T-r_{*}(R)=\text { const. }
$$

where $T$ and $R$ are the Schwarzschild time and areal radius coordinates, respectively, $r_{*}$ is the Schwarzschild tortoise coordinate

$$
r_{*}(R)=R+2 M_{\mathrm{ADM}} \ln \left(\frac{R}{2 M_{\mathrm{ADM}}}-1\right)
$$

and $M_{\mathrm{ADM}}$ is the mass of the Schwarzschild spacetime. The binary black-hole spacetime and its coordinates $t, r$ approach Schwarzschild as $r \rightarrow \infty$, so we approximate the null geodesics of the binary black-hole spacetime using 25 and 26. with $r \approx R, t \approx T\lceil\dagger \dagger$, and will extrapolate along curves of constant $u$.

$\dagger \dagger \dagger$ All coordinate systems used by the numerical-relativity codes are expected to satisfy the property $r \rightarrow R, t \rightarrow T$ as $r \rightarrow \infty$. The error incurred by evaluating at a finite radius will be included in the measure of extrapolation error (see Sec. 5.2. Better approximations here can take into account numerical measures of the lapse and areal radius, for example, and lead to reduced errors in the extrapolation 214. 
The waveforms are computed in the numerical codes at coordinate times which have no relation to the $u=$ const. curves needed for extrapolation. We choose a set of retarded time-values $u_{i}$, and compute for each radius and each $u_{i}$ the corresponding coordinate time $t_{i}(r)=u_{i}+r_{*}(r)$. For each radius $r$, the finite-radius waveforms are interpolated to the coordinate times $t_{i}(r)$. For certain gauge choices, such as the damped harmonic gauge used in SpEC simulations, increased accuracy is obtained by including corrections to (25) that stem from the relatively strong variation of the lapse and areal radius, especially during merger (see 214 for details). We do not use this advanced extrapolation method in this paper to maintain uniformity of the analysis between simulations from different groups.

The real and imaginary parts of the $H_{\ell m}$ coefficients of a waveform oscillate with each gravitational-wave cycle. The complex amplitude and phase of these modes show less structure. These quantities are given by

$$
\begin{aligned}
A_{\ell m}\left(u_{i}, r\right) & =\left|H_{\ell m}\left(t_{i}(r), r\right)\right|, \\
\phi_{\ell m}\left(u_{i}, r\right) & =\arg H_{\ell m}\left(t_{i}(r), r\right)+2 \pi n,
\end{aligned}
$$

where $n$ is determined by continuity of $\phi_{\ell m}\left(u_{i}, r\right)$ in $u$. Extrapolating quantities without oscillatory behavior (i.e. $A$ and $\phi$ ) reduces extrapolation errors significantly. For each retarded time $u_{i}$, and each spherical harmonic mode $(\ell, m)$, we perform two separate linear least squares fits of the form

$$
f\left(u_{i}, r\right)=\sum_{n=0}^{p} \frac{a_{n}}{r^{n}} .
$$

The left-hand-side of $(29)$ represents either $r$ times the amplitude, $r A_{\ell m}\left(u_{i}, r\right)$, or the phase, $\phi_{\ell m}\left(u_{i}, r\right)$, at all extraction radii $r$ at the target retarded time $u_{i}$. The right-hand-side is the fitting polynomial with fitting coefficients $a_{n}$.

The leading order coefficient $a_{0}$ is then taken as the value extrapolated to $\mathcal{J}^{+}$ $(r \rightarrow \infty)$ at order $p$. All radii at which the whole waveform is sufficiently resolved in the numerical simulation are included in the extrapolation. We denote the waveform extrapolated in this manner as $\mathcal{R}_{p}\left(H_{\ell m}\right)$. As an exceptional case, when $p=0$ (no extrapolation $), a_{0}$ is taken to be $f\left(u_{i}, r_{\max }\right)$ instead of the result of the fit.

An alternative extrapolation method consists of using the results of perturbation theory to propagate waveforms obtained at finite radius (but in the radiation zone) to $\mathcal{J}^{+}$. A simple explicit formula can be found relating $\Psi_{4}$ at $\mathcal{J}^{+}$with the finite radius $\Psi_{4}$ and its time integral. For more details, see (53) in 206. This method has been shown to be correct for the next-to-leading $1 / r$ term in $r \Psi_{4}(r, t)$ using only a single observer radius and displays a significantly reduced level of extrapolation noise 62, 215]. The errors produced by this method can be estimated by applying it to different extraction radii. We applied this method to the $q=10$ case and found good agreement (but with significantly reduced noise) between the perturbative technique and the standard extrapolation technique described above.

\subsection{Error analysis}

We identify and provide estimates for three distinct sources of error in the numericalrelativity waveforms:

(i) Finite numerical resolution; 
(ii) Waveform measurement at finite distance from the source;

(iii) Computation of $h$ from $\Psi_{4}$.

Finite resolution error results from the conversion of the continuum Einstein equations into a discrete form suitable for numerical solution. The accuracy of the solution can be increased at the expense of computational cost by decreasing the grid spacing between the numerical grid points on which the solution is represented (in the case of spectral codes, these are the collocation points of the basis functions). One can thus obtain an error estimate by considering two such numerical solutions and the theoretically-expected behaviour of the uncontrolled remainder terms. However, these terms are difficult to model, since within a single simulation, many grid-dependent approximations are used, and the resulting errors combine in complicated ways. For each type of code, finite-difference and spectral, an estimate is made of the dominant source of error and its convergence properties, and this is used to estimate the error in the solution.

For finite-difference codes, the error is estimated using Richardson extrapolation assuming polynomial convergence of the solution at order $r$. The choice of $r$ depends on the approximations used in a given code, and is chosen by the NR group. A quantity $q$ which converges at order $r$ with the grid spacing $\delta$ satisfies

$$
q(\delta)=q_{0}+C \delta^{r}+O\left(\delta^{r+1}\right),
$$

where $q_{0}$ (the continuum solution) and $C$ are unknown and independent of $\delta$. Taking two numerical solutions with different grid spacings $\delta$, the resulting simultaneous equations are solved for $q_{0}$ and $C$, and the error in $q\left(\delta_{2}\right)$ due to truncation of the finite difference approximation is

$$
\sigma_{\mathrm{T}}(q) \equiv q\left(\delta_{2}\right)-q_{0}=\frac{q\left(\delta_{2}\right)-q\left(\delta_{1}\right)}{1-\left(\delta_{1} / \delta_{2}\right)^{r}}+O\left(\delta^{r+1}\right), \quad \text { FD codes. }
$$

Note that (31) assumes that the error indeed decays like $\delta^{r}$, and that the higher order terms $O\left(\delta^{r+1}\right)$ are negligible.

For spectral codes in simple situations (a single domain, no time integration, etc), the error is formally exponentially convergent in the number of grid points. However, due to the complexity of the typical grids employed, and the mix of different sources of error, this exponential convergence is usually not observed. In tests where the exact solution is known, the error typically behaves such that $\sigma_{\mathrm{T}}\left(q_{2}\right) \ll \sigma_{\mathrm{T}}\left(q_{1}\right)$ if $\delta_{2}<\delta_{1}$ for solutions $q_{1}=q\left(\delta_{1}\right)$ and $q_{2}=q\left(\delta_{2}\right)$ for typical choices of grid spacings $\delta_{1}$ and $\delta_{2}$. Since $\sigma_{\mathrm{T}}\left(q_{1}\right)=q_{1}-q_{2}+\sigma_{\mathrm{T}}\left(q_{2}\right)$, then if $\sigma_{\mathrm{T}}\left(q_{2}\right)$ can be neglected, $\sigma_{\mathrm{T}}\left(q_{1}\right)=q_{1}-q_{2}$ is an error estimate on $q_{1}$. Since $\sigma_{\mathrm{T}}\left(q_{1}\right) \gg \sigma_{\mathrm{T}}\left(q_{2}\right)$, this also serves as a conservative bound on the error of $q_{2}$, and is what we quote as its error:

$$
\sigma_{\mathrm{T}}(q) \equiv q\left(\delta_{2}\right)-q\left(\delta_{1}\right), \quad \operatorname{SpEC} .
$$

Since small dephasings can lead to large errors in $\operatorname{Re}[h]$ and $\operatorname{Im}[h]$, given complex waveforms $h=A \exp i \phi$ computed at different grid spacings, we quote the errors $\sigma_{\mathrm{T}}(A)$ and $\sigma_{\mathrm{T}}(\phi)$ rather than $\sigma_{\mathrm{T}}(h)$, as this yields a more useful measurement. The error is clearly identified as a dephasing rather than a mixture of phase and amplitude errors. 
Table 4. Extrapolation orders $p$ chosen for the amplitude $(A)$ and phase $(\phi)$ for each waveform.

\begin{tabular}{llll}
\hline$\#$ & Label & $p(A)$ & $p(\phi)$ \\
\hline $12-13$ & A-* & 2 & 2 \\
$16-24$ & S-* & & \\
\hline 3 & F1p+30-30 & 0 & 1 \\
9 & R10 & & \\
11 & L3+60+00 & & \\
\hline Others & & 2 & 1 \\
\hline
\end{tabular}

Finite radius error results from the computation of gravitational radiation at a finite distance from the source instead of computing it at future null infinity. All waveforms except those computed using CCE make this approximation. To reduce this error, all waveforms are extrapolated from several finite radii as described in Sec. 5.1 .

Due to the different choices of extraction radii made by each group for each waveform, the optimal extrapolation order $p$ varies across the data set. Using a lower extrapolation order leads to a larger error, but using too high an extrapolation order leads to unwanted overfitting of a model with a large number of degrees of freedom in comparison to the number of data points. We found it difficult to construct a strict automated criteria for choosing $p$, and instead visually inspected the behaviour of fits to $A(u, r)$ and $\phi(u, r)$ as functions of $r$ at several values of $u$. We judged whether a given order of extrapolation led to a reasonable fit given the number of radii and the distance over which the extrapolation in $1 / r$ was performed relative to the difference between the minimum and maximum radius, and whether it was possible to construct a good error estimate. Table 4 shows the extrapolation orders chosen for each waveform. Generally, second order extrapolation was possible for $A$ for most of the waveforms, and first order for $\phi$. Waveforms computed at high radius $r>200 M$ were suitable for higher order extrapolation in $\phi(p=2)$. For three cases, the behaviour of $A$ with radius led us to perform no extrapolation in $A$ (denoted $p=0$ ) and simply use the value from the largest extraction radius.

The error due to finite-radius effects is estimated by comparing the extrapolant at different orders. The error in the order $p$ extrapolant, $\mathcal{R}_{p}(q)$, is estimated as

$$
\sigma_{\mathrm{R}}(q) \equiv \min \left(\left|\mathcal{R}_{p}(q)-\mathcal{R}_{p+1}(q)\right|+\left|\mathcal{R}_{p+1}(q)-\mathcal{R}_{p+2}(q)\right|,\left|\mathcal{R}_{p}(q)-\mathcal{R}_{0}(q)\right|\right),
$$

where $q \in\{A, \phi\}$. There are two contributions to this error estimate. If extrapolation works well, i.e. different extrapolation orders do not change the results significantly, then we trust the extrapolation, and take the variation with extrapolation order as indicative of the error. We take the sum of the variations from the next two extrapolation orders, to guard against coincidentally small changes, and to ensure that extrapolation works through at least two more orders. If extrapolation fails (e.g. if higher order extrapolation fits to likely-unphysical features in the different extraction radii), then the tentative error computed by this method will be very large. The large value is caused by the insufficient fit, and is not a reliable estimate of the extrapolation error. In this case, the difference with the outermost extraction radius is used $(p=0)$. Taking the minimum is a means to automatically switch between these two methods. 
Strain error results from the computation of $h$ from $\Psi_{4}$. The FFI method introduces errors caused by spectral leakage and insufficiently low choices of cutoff frequency (see [211]). We generally expect the cutoff frequency to be high enough that spectral leakage is not an issue, and estimate the error due to the choice of cutoff frequency by differentiating the strain twice to get $\Psi_{4}$, and comparing this with the original $\Psi_{4}$. If there was no loss of signal due to the cutoff, we would obtain exactly the original $\Psi_{4}$. Hence, we use the difference as an indication of the effects of the FFI error, and quote phase and relative amplitude errors measured in $\Psi_{4}$ here as if they were errors on the resulting $h$,

$$
\begin{aligned}
\sigma_{\mathrm{S}}(\phi) & \equiv \arg \left(\Psi_{4}\right)-\arg (\ddot{h}), \\
\frac{\sigma_{\mathrm{S}}(A)}{A} & \equiv \frac{\left|\Psi_{4}\right|-|\ddot{h}|}{|\ddot{h}|} .
\end{aligned}
$$

Better methods for estimating the error due to FFI may be developed in the future.

Non-aligned and aligned error estimates: In (31)-(35), we use differences between two waveforms to estimate the error arising from a certain effect. When computing these differences, we pursue two approaches: first, we compute the differences as stated, as functions of time $t$, where the same $t$ is used in both waveforms. This is a non-aligned difference, and directly measures the error in the simulation.

The second approach incorporates alignment of the two waveforms being compared. Because gravitational-wave detection, and matching with analytical models, always extremize over the time-of-arrival and phase of the gravitational waveform, the accuracy of these two degrees of freedom is less important for those applications. The effect of these two degrees of freedom can be eliminated by timeand phase-shifting one of the two waveforms being compared relative to the other, so as to minimize a measure of their difference.

In practice, we choose an alignment window $\left[t_{1}, t_{2}\right]$, hold one waveform $(A)$ fixed, while time- and phase-shifting the other $(B)$ to minimize $31,128,131$

$$
\Xi(\delta t, \delta \Phi)=\int_{t_{1}}^{t_{2}}\left[\phi_{22}^{(A)}(t)-\phi_{22}^{(B)}(t+\delta t)-2 \delta \Phi\right]^{2} d t .
$$

For a given $\delta t$, the minimization over $\delta \Phi$ can be done analytically, reducing the problem to a one-dimensional minimization 31. Waveform $B$ is then shifted in time and phase accordingly; the phase of any $(\ell, m)$ mode is shifted by $m \delta \Phi$. Note that this transformation is appropriate for rotations about the $z$ axis. In significantly precessing systems, alignments will require more general rotations 49,216 218].

The choice of alignment window is as much art as science [35,131], but is chosen to be the same as the window that would be used when aligning the analytic waveform to the numerical waveform (though such alignment is not performed in this paper). Thus, the resulting error measure will describe the precision to which the analytical and numerical waveforms can be expected to agree.

Combined error estimates: The three sources of error - truncation, finite radius and fixed-frequency integration - each contribute to the total error in the waveform. Each one is computed individually without and with alignment. We add them in quadrature 


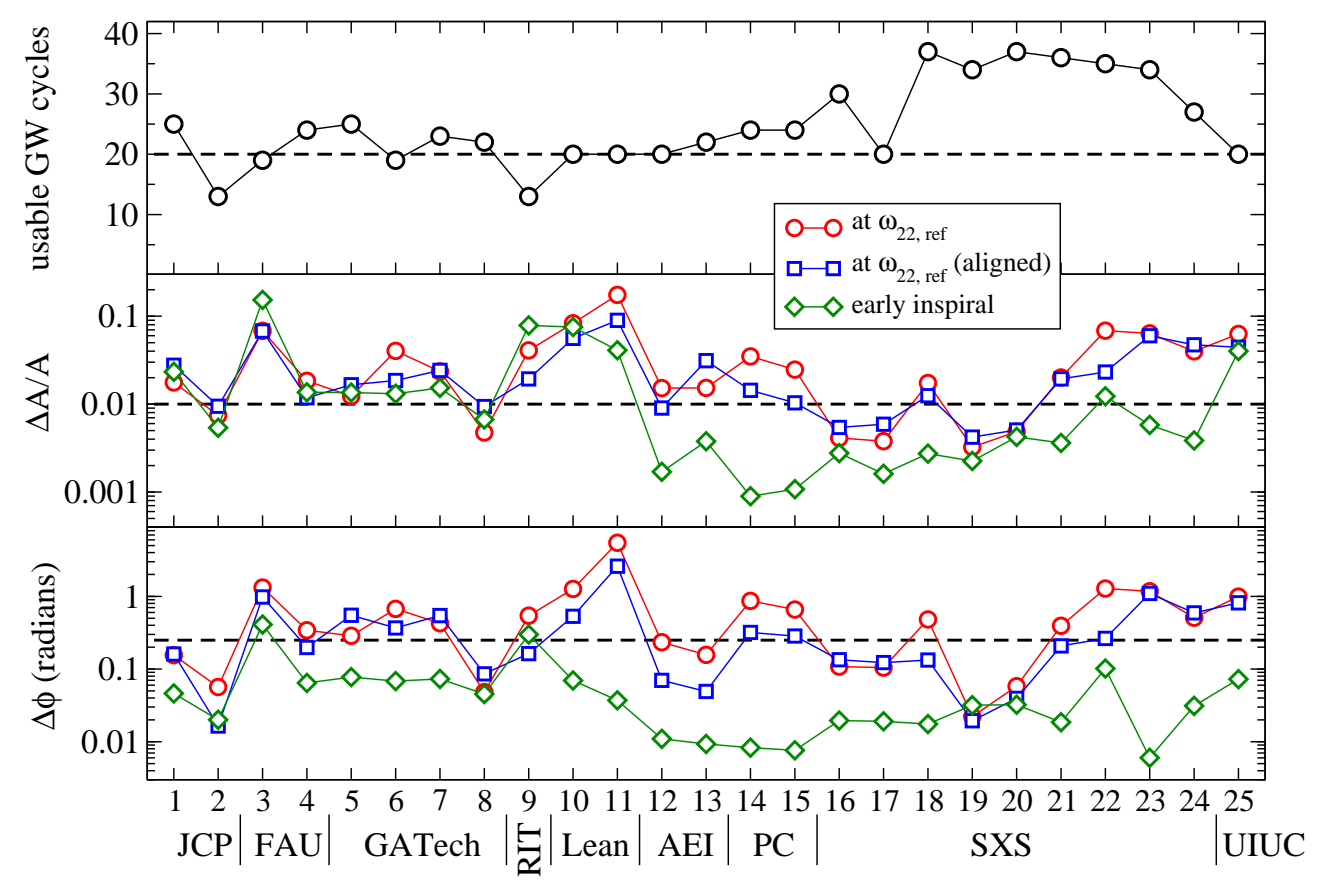

Figure 4. Error estimates for the numerical simulations. The top panel shows the usable gravitational-wave cycles of each waveform, the middle panel the relative amplitude error of the $(2,2)$ mode, and the bottom panel the phase error of the $(2,2)$ mode. The horizontal axis indicates the case-number and the group name (cf. table 1). The dashed lines in each panel indicate the length and accuracy goals.

to obtain our final error estimates on the amplitude and phase of the strain waveform $h$,

$$
\sigma(q)=\sqrt{\sigma_{\mathrm{T}}(q)^{2}+\sigma_{\mathrm{R}}(q)^{2}+\sigma_{\mathrm{S}}(q)^{2}},
$$

where $q \in\{A, \phi\}$. Here, (37) is evaluated separately for the non-aligned and aligned differences, for a total of four time-series $\sigma(q)$ per waveform. Finally, each of these time-series is further reduced to individual real numbers by evaluation at both $t_{0}$ and $t_{\text {ref }}$ (recall that $t_{0}$ is the time after which the effects of junk-radiation are no longer visible in the waveform, and $t_{\text {ref }}$ is the time at which the gravitational-wave frequency of the $(2,2)$ mode $M \omega_{\mathrm{GW}}=M \omega_{22}$, ref $\left.\equiv 0.2\right)$. These reduced error estimates are plotted in figure 4 for all waveforms. Appendix B contains these data in numerical form in table B1

Comparison with targets: We now assess, with reference to figure 4 , the degree to which the waveforms meet the accuracy and length targets described in Sec. 3.2 . All waveforms contain $\gtrsim 20$ usable gravitational wave cycles, apart from two, including Case 9 (R10), which requires great computational cost due to the high mass ratio $(q=10)$. 19 out of 25 waveforms have a relative amplitude error $\lesssim 1 \%$ during the early inspiral, but all have a phase error $\lesssim 0.25$ radians during the early inspiral. The error at early times is dominated by the effects of finite-radius wave extraction, as numerical phase error in the motion of the binaries has not yet accumulated. All waveforms allow at least first order extrapolation with radius in the phase, and this 

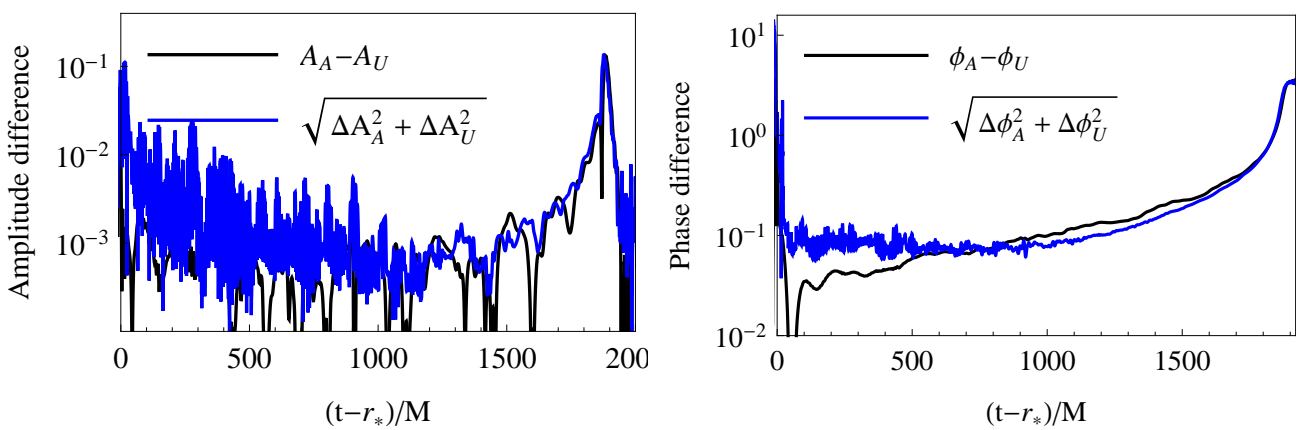

Figure 5. Comparison of the difference between the amplitudes (left) and phases (right) of the waveforms $12(\mathrm{~A} 1+30+00)$ and $25(\mathrm{U} 1+30+00)$ and their combined error estimates. The error estimates are comparable with the disagreement between the waveforms, indicating that the results are compatible with each other. (The error estimates are not rigourous bounds, hence they do not always cover the differences completely.)

is shown to be sufficient to meet the accuracy targets. Amplitude errors during the early inspiral are typically caused by wave extraction at too low a radius, or with too few radii, possibly due to limitations of computational cost specific to certain codes or binary configurations. At the reference time shortly before the merger, 16 out of 25 waveforms have a relative amplitude error $\lesssim 1 \%$ and 17 out of 25 waveforms have a phase error $\lesssim 0.25$ radians (we quote results for the aligned errors). Here the error is typically dominated by the effects of insufficient resolution, as numerical truncation error accumulates with time, especially for the phase.

While not all waveforms meet the accuracy targets, we chose to include all of them in the comparison with analytical models with the exception of Case 11, which has errors at merger significantly larger than any of the other waveforms. The results in Sec. 6 are presented with error bars computed from these error estimates.

Consistency check: Two of the waveforms (Cases $12(\mathrm{~A} 1+30+00)$ and 25 $(\mathrm{U} 1+30+00))$ were computed from the same spatial initial data using two different codes. As such, they should agree within the quoted error estimates. Figure 5 shows the difference between the amplitude and phase of each waveform compared to the corresponding error estimate. The error estimates are comparable with the differences, indicating that the results are compatible.

\section{Comparison of numerical waveforms with existing analytical templates}

We now compare the numerical waveforms $h_{\mathrm{NR}}$ produced in this paper with analytical waveforms $h_{\mathrm{AR}}$ generated by existing spinning non-precessing template models the time-domain SEOBNRv1 model 87 and the frequency-domain phenomenological IMRPhenomB 91 and IMRPhenomC 92 models. In the $q=10$ non-spinning case, we also compare with analytical waveforms generated by two non-spinning EOB models - the EOBNRv2 model [219], which has been used in the searches of gravitational waves from coalescing binary black holes with LIGO and Virgo [94, 95, and the recently developed IHES-EOB model 88 . The comparisons serve as both a sanity check of the numerical waveforms and an evaluation of the analytical models. Furthermore, they provide indications of whether the numerical waveforms produced 
in this paper can be used to improve the existing analytical models. We stress that none of the above analytical models were calibrated using the numerical waveforms produced by the NRAR collaboration. However, the SEOBNRv1 model was calibrated in 87 to two waveforms independently generated by the SXS collaboration and later contributed to the NRAR collaboration, specifically Case $16(\mathrm{~S} 1+44+44)$ and Case 17 (S1-44-44) in table 1 .

As described above, we restrict our analysis here to the $\ell=2, m=2$ mode of the gravitational waveforms. We measure the difference between numerical and analytical waveforms with the unfaithfulness 220.

$$
\overline{\mathcal{F}} \equiv 1-\max _{t_{c}, \phi_{c}} \frac{\left\langle h_{\mathrm{NR}}, h_{\mathrm{AR}}\right\rangle}{\sqrt{\left\langle h_{\mathrm{NR}}, h_{\mathrm{NR}}\right\rangle\left\langle h_{\mathrm{AR}}, h_{\mathrm{AR}}\right\rangle}}
$$

and the ineffectualness 220.

$$
\overline{\mathcal{E}} \equiv 1-\max _{t_{c}, \phi_{c}, \vec{\lambda}} \frac{\left\langle h_{\mathrm{NR}}, h_{\mathrm{AR}}\right\rangle}{\sqrt{\left\langle h_{\mathrm{NR}}, h_{\mathrm{NR}}\right\rangle\left\langle h_{\mathrm{AR}}, h_{\mathrm{AR}}\right\rangle}},
$$

where we denote the time and phase of coalescence of $h_{\mathrm{AR}}$ by $t_{c}$ and $\phi_{c}$, and the binary parameters of $h_{\mathrm{AR}}$ by $\vec{\lambda}$. The dependence of $h_{\mathrm{AR}}$ on $t_{c}, \phi_{c}$ and $\vec{\lambda}$ has been omitted for brevity. We define the inner product between two waveforms through the following integral in the frequency domain

$$
\left\langle h_{1}, h_{2}\right\rangle \equiv 4 \operatorname{Re} \int_{0}^{\infty} \frac{\tilde{h}_{1}(f) \tilde{h}_{2}^{*}(f)}{S_{h}(f)} d f,
$$

where $\tilde{h}_{1}(f)$ and $\tilde{h}_{2}(f)$ are frequency-domain waveforms

$$
\tilde{h}_{k}(f)=\int_{-\infty}^{\infty} h_{k}(t) \mathrm{e}^{-2 \pi \mathrm{i} f t} d t \quad(k=1,2)
$$

and $S_{h}(f)$ is the noise power spectral density of the detector. In this paper, we employ the zero-detuned high-power advanced LIGO noise curve ZERO_DET_HIGH_P [102]. The unfaithfulness of analytical waveforms is the normalized inner product minimized over $t_{c}$ and $\phi_{c}$ and it is related to the bias in measuring the binary parameters. The ineffectualness is also minimized over $\vec{\lambda}$ and quantifies the efficiency in detecting gravitational-wave signals. Although here we are mainly interested in understanding whether the existing template families are effectual in detecting the numerical waveforms given in table 1. we also want to study the consistency of these models against the numerical waveforms over the entire frequency range. This will tell us what region future improvements should focus on. Thus, both unfaithfulness and ineffectualness give us important, complementary information. Because it is computationally expensive to minimize over $\vec{\lambda}$ when using the time-domain EOB models, all the plots in this section will show the unfaithfulness, and for only the few cases for which the unfaithfulness is larger than $1 \%$, we calculate the ineffectualness.

The definition of $\left\langle h_{1}, h_{2}\right\rangle$ given in 40 involves an integral over frequency from 0 to $\infty$. In reality, since the noise power spectral density of the detector has a sharp low-frequency cutoff due to seismic noise, it is safe to start the integral at this cutoff frequency, which is $10 \mathrm{~Hz}$ for the ZERO_DET_HIGH_P noise curve of the advanced LIGO detector. However, for a reasonable range of the total mass $M$ of 


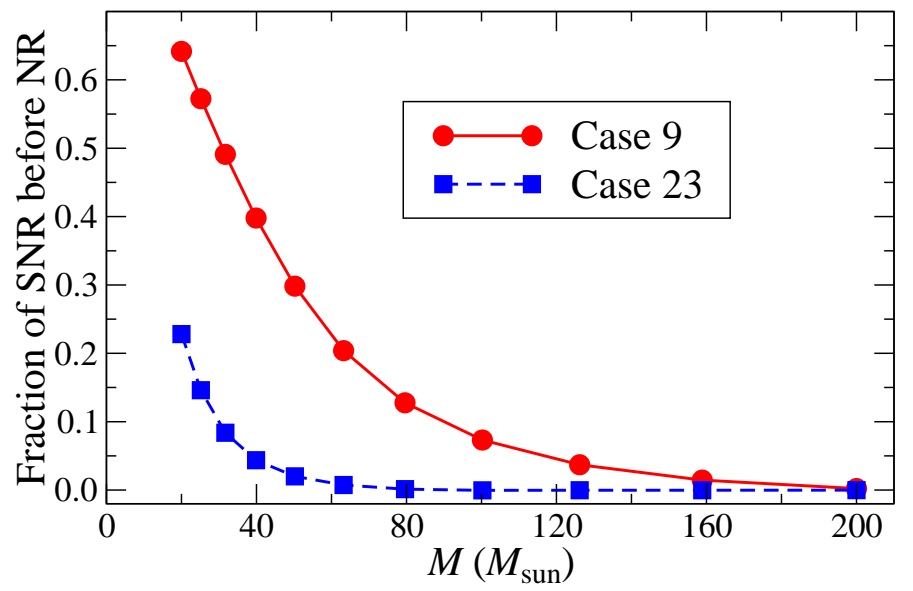

Figure 6. Fraction of SNR accumulated before the numerical waveform starts. For NR-only overlap calculations, we begin the overlap integral given in 40 at the frequency where the numerical waveform begins, ignoring the fraction of SNR shown in this figure, which would be accumulated at lower frequencies. The losses shown in this figure are calculated using SEOBNRv1 waveforms for binary configurations $9(\mathrm{R} 10)$ and $23(\mathrm{~S} 1 \mathrm{p}-30-30)$ in table 1 The low-frequency truncations are at $\left(220 M_{\odot} / M\right) \times 10 \mathrm{~Hz}$ and $\left(90 M_{\odot} / M\right) \times 10 \mathrm{~Hz}$ for Cases 9 and 23 , respectively.

a binary system, which we choose to be $20 M_{\odot}-200 M_{\odot}$ in this section, the numerical waveforms do not always have a small enough initial dimensionless frequency to start at a physical frequency of $10 \mathrm{~Hz}$. In fact, the numerical waveform with the largest initial (dimensionless) frequency (Case 9 (R10)) starts at $\left(220 M_{\odot} / M\right) \times 10 \mathrm{~Hz}$, while the one with the smallest initial frequency (Case $23(\mathrm{~S} 1 \mathrm{p}-30-30)$ ) starts at $\left(90 M_{\odot} / M\right) \times 10 \mathrm{~Hz}$ (see also (1) above and discussion around it). Here we do not build hybrid waveforms by joining together numerical-relativity waveforms to $\mathrm{PN}$-approximants 99 , because we are interested in assessing the closeness of the analytical waveforms to numerical waveforms and do not want to spoil the result by introducing errors due to the disagreement between the analytical waveforms and the PN-approximants used to build the hybrid waveforms.

To reduce artefacts when the numerical waveforms start in the detector bandwidth, we truncate the analytical waveforms in the time domain when they reach the starting frequency of the numerical waveform and taper all waveforms using the Planck-taper window function 221. The width of the window function is set to the length of the numerical waveforms, starting from $t=t_{0}$, after which the effects of junk radiation are no longer important. The window function smoothly rises from 0 to 1 in the first $200 M$ and falls from 1 to 0 in the last $20 M$ of the waveforms. The unphysical junk radiation is therefore completely excluded from the comparison. For the integral in 400, we choose as our low-frequency cutoff $f_{\text {low }}$ the frequency at which the numerical waveforms start. This low-frequency cutoff is chosen separately for each numerical waveform, and can be as high as $110 \mathrm{~Hz}$. Since these low-frequency cutoffs are substantially inside the sensitivity range of the ZERO_DET_HIGH_P noise curve used here, we must be careful when interpreting the unfaithfulness and ineffectualness results.

In the absence of longer numerical waveforms, we cannot exactly quantify the 
effect of choosing the starting frequency of the numerical waveform as our lowfrequency cutoff. However, we can at least investigate and estimate the effect of neglecting the SNR below the starting frequency of the numerical waveform. In figure 6 we show the fraction of SNR lost due to the low-frequency cutoff for the two numerical waveforms with the lowest and highest starting frequencies. Figure 6 is computed using SEOBNRv1 waveforms having the same physical parameters as the numerical waveforms. We can see that for the waveform with the lowest initial frequency (Case $23(\mathrm{~S} 1 \mathrm{p}-30-30))$, the loss of SNR is less than a quarter when $M$ is above $20 M_{\odot}$ and less than $1 \%$ when $M$ is above $100 M_{\odot}$, indicating that the amount of signal lost by truncating the waveforms is relatively small. We therefore expect the results calculated from the low-frequency truncated integrals to be reliable estimates of the exact unfaithfulness and ineffectualness, at least when $M$ is above $100 M_{\odot}$. For the waveform with the highest initial frequency (Case 9 (R10)), however, the loss of SNR can be more than half when $M$ is around $20 M_{\odot}$. Thus, in this case, the unfaithfulness and ineffectualness results calculated from the low-frequency truncated integrals do not allow us to draw definitive conclusions about whether the analytical waveforms are sufficiently accurate for detection and/or measurement purposes for low-mass binaries. Nevertheless, we believe that even when the loss of SNR is high, say $\gtrsim 10 \%$, as in Case $9(\mathrm{R} 10)$ for $M \leq 100 M_{\odot}$, the results obtained with the lowfrequency truncated integrals are still meaningful for understanding and improving the modeling errors in the frequency band covered by the current numerical waveforms.

The reason is the following. When the total mass is $100 M_{\odot}\left(200 M_{\odot}\right)$, the numerical waveform for Case 9 (R10) covers a frequency range of $22-135 \mathrm{~Hz}(11-$ $68 \mathrm{~Hz})$ and the detector noise power spectral density $S_{h}(f)$ decreases by more than one (two) order(s) of magnitude going from the low end to the high end of the frequency range. Thus, when $M \geq 100 M_{\odot}$, the unfaithfulness and ineffectualness results are particularly sensitive to the differences between the numerical and analytical waveforms at high frequency during the merger-ringdown stage. On the contrary, when the total mass is $20 M_{\odot}, S_{h}(f)$ changes by less than a factor of 1.5 in the frequency range of $110-680 \mathrm{~Hz}$ and the unfaithfulness and ineffectualness results provide us with a broader measure of the overall agreement of the numerical and analytical waveforms during inspiral, merger and ringdown. Thus, when the total mass decreases from $100 M_{\odot}$ to $20 M_{\odot}$, the unfaithfulness and ineffectualness are increasingly more sensitive to modeling errors at lower frequency. If the unfaithfulness and ineffectualness are high, it means that the analytical modeling of the last stages of inspiral (i.e. 20-30 gravitational-wave cycles before merger) is not very reliable. In summary, unfaithfulness and ineffectualness results computed with the low-frequency truncated integral may not be used to draw definitive conclusions for detection and parameterestimation purposes when $M \leq 100 M_{\odot}$, but they do provide us with important information on the modeling errors of the analytical waveforms during the last stages of inspiral, and can guide us in improving the models in the future.

Finally, we have carried out the following test to further check the use of the low-frequency truncated integral. We have applied the procedure proposed in 130] and have extended both the numerical and analytical waveforms (SEOBNRv1 model) at low frequency attaching to them the same waveform, so that they span the entire frequency band of advanced LIGO. Using those extended waveforms, we have computed the unfaithfulness and compared it with the unfaithfulness derived with the low-frequency truncated integral. We have found very small differences between these two methods that have no impact on any conclusion of this paper. Therefore, we 

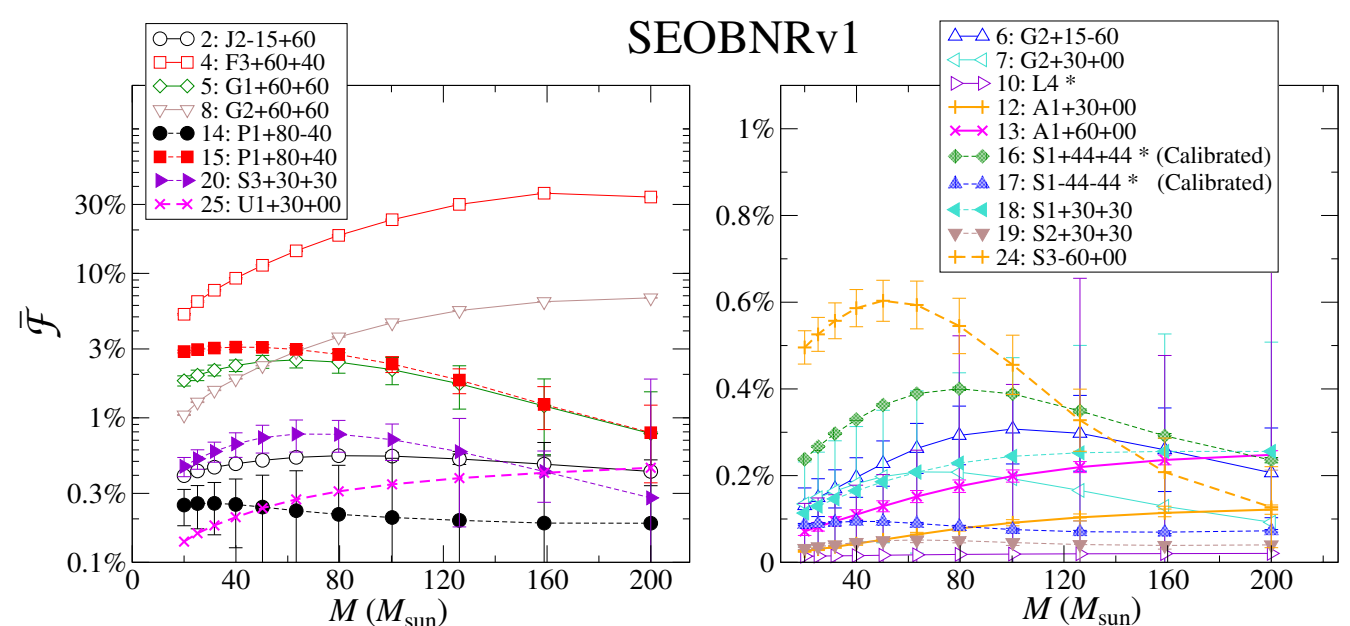

Figure 7. Unfaithfulness $\overline{\mathcal{F}}$ of the SEOBNRv1 waveform model compared to the numerical simulations. The horizontal axis shows the total mass, the vertical axis $\overline{\mathcal{F}}$ in percent. The waveforms are split between the two panels for readability. Note that for $M \lesssim 100 M_{\odot}$ the plot disregards the low-frequency portion of the waveform which is below the starting frequency of the numerical waveform yet of substantial contribution to the SNR (cf. Sec.6 and figure 6). Numerical relativity error-estimates $\delta \overline{\mathcal{F}}$ on the computed unfaithfulness values are plotted, except for Cases 18 and 25 for which $\delta \overline{\mathcal{F}}>20 \%$. Note that the SEOBNRv1 model was calibrated to the contributed Cases $16(\mathrm{~S} 1+44+44)$ and $17(\mathrm{~S} 1-44-44)$ shown in the right panel with shaded symbols.

henceforth restrict our discussion to the unfaithfulness and ineffectualness computed with the low-frequency truncated integral for total masses $20-200 M_{\odot}$.

\subsection{Results using the effective-one-body waveform models}

Of the 25 waveforms presented in table 1, 5 are precessing. In figure 7 , for 18 out of the remaining 20 non-precessing configurations, we show the unfaithfulness between the numerical waveforms and the analytical SEOBNRv1 waveforms as functions of the total mass. We show the unfaithfulness of Case 9 (R10) in figure 8 and omit Case $11(\mathrm{~L} 3+60+00)$, due to its large numerical errors (see figure 4 and table B1).

The SEOBNRv1 model is a non-precessing spin model. It was calibrated in 87 to five non-spinning numerical waveforms with mass ratios $1,2,3,4$ and 6 [66 and two equal-mass spinning, aligned/antialigned numerical waveforms (the contributed waveforms Cases $16(\mathrm{~S} 1+44+44)$ and $17(\mathrm{~S} 1-44-44))$. The SEOBNRv1 model was extrapolated to any mass ratio and to dimensionless spin values $\chi$ in the range -1 to 0.7 , using inspiral, merger and ringdown waveforms produced with a Teukolskyequation code in the large mass-ratio limit 109].

By definition of the unfaithfulness, the binary parameters of the signal and the template coincide. So, when comparing SEOBNRv1 and numerical-relativity waveforms, we evaluate the SEOBNRv1 waveforms using the binary parameters extracted from the numerical simulations immediately after the junk radiation. Whilst these parameters are somewhat gauge dependent, and we have not rigorously included error estimates for them, the good agreement with EOB shown below indicates that this is not a major source of error. For clarity, we divide the results into two panels 
(see figure 7). In each panel, we also show the numerical errors, which we compute as the unfaithfulness $\delta \overline{\mathcal{F}}$ between a numerical waveform $h_{\mathrm{NR}}=A \mathrm{e}^{\mathrm{i} \phi}$ and the waveform $h_{\mathrm{NR}, \Delta}=(A \pm \Delta A) \mathrm{e}^{\mathrm{i}(\phi \pm \Delta \phi)}$, where $\Delta A$ and $\Delta \phi$ are the numerical amplitude and phase errors estimated in Sec. 5.2 .

As can be seen in figure 7, at the low-mass end, in all 18 Cases, the unfaithfulness is at most a few percent, with slopes always positive, indicating increasingly better agreement with the numerical-relativity waveforms at low frequency during the last stages of inspiral. At the high-mass end, in 16 out of 18 cases the unfaithfulness is below $1 \%$, indicating a highly faithful modeling of the merger-ringdown phase in the SEOBNRv1 model.

In Cases 8 and especially 4, the unfaithfulness is much larger than that of the other cases, especially when the total mass is high. We find that in these cases, the amplitudes and frequencies of the SEOBNRv1 waveforms have artificial oscillations around merger. Although these oscillations appear tiny in the time domain, they become much more significant in the frequency domain and lead to the substantial increase of unfaithfulness. It is interesting that this study with the NRAR waveforms has uncovered those spurious features in the EOB model that will be corrected in future modeling. We recall that the SEOBNRv1 model was calibrated to five nonspinning numerical simulations $(1 \leq q \leq 6)$ and only two spinning non-precessing simulations with equal masses and equal, mild $(|\chi|=0.44)$ spin components. The input values 87] that are extracted from the numerical waveforms and used to reshape the EOB waveform around merger were extrapolated across the entire parameter space using only a handful of numerical simulations. Thus, it is not surprising that when we extrapolate the SEOBNRv1 waveforms to unequal-mass and/or unequalspin configurations that are quite far from the calibrated points, we can observe some artefacts around merger. Although the unfaithfulness is large in these two cases (up to $30 \%$ in Case 4), the ineffectualness is much smaller. We find that by minimizing only over the binary component masses the ineffectualness can already be reduced to below $2 \%$. We expect a further reduction when minimizing also over the black-hole spins. Since we estimate that the ineffectualness in all 18 cases is below $1 \%$, we conclude that for total masses $\geq 100 M_{\odot}$ the SEOBNRv1 waveforms are sufficiently accurate for detection purposes, i.e. the modeling error will cause a loss in event rates smaller than $3 \%$.

Five cases in table1 (viz. 1, 3, 21, 22 and 23), are spinning, precessing waveforms and are not included in figure 7 . Since the mass ratios for these cases are not very large, $q=3$ for Case 21 and $q=1$ for the other four, precession-induced modulations are quite mild. Nevertheless, it is interesting to check whether the nonprecessing SEOBNRv1 model is able to match mildly-precessing numerical waveforms. To compare the waveforms in the presence of precession, at the initial time of the numerical simulation we set the EOB spin components in the orbital plane to zero and we identify the numerical and EOB spin components along the direction perpendicular to the orbital plane. Using non-precessing waveforms generated with these EOB spins, we find that the unfaithfulness in all five cases is a few percent at low total masses and below $1 \%$ at high total masses. The non-precessing SEOBNRv1 waveforms are therefore good approximations to the mildly precessing numerical waveforms produced in this paper. It is worth noting that in Cases 22 and 23, the unfaithfulness has a negative slope toward low total masses. This is not surprising, since we expect that relatively stronger precessional effects accumulate during the inspiral, and such effects are not included in the non-precessing SEOBNRv1 model. It is worth pointing out 


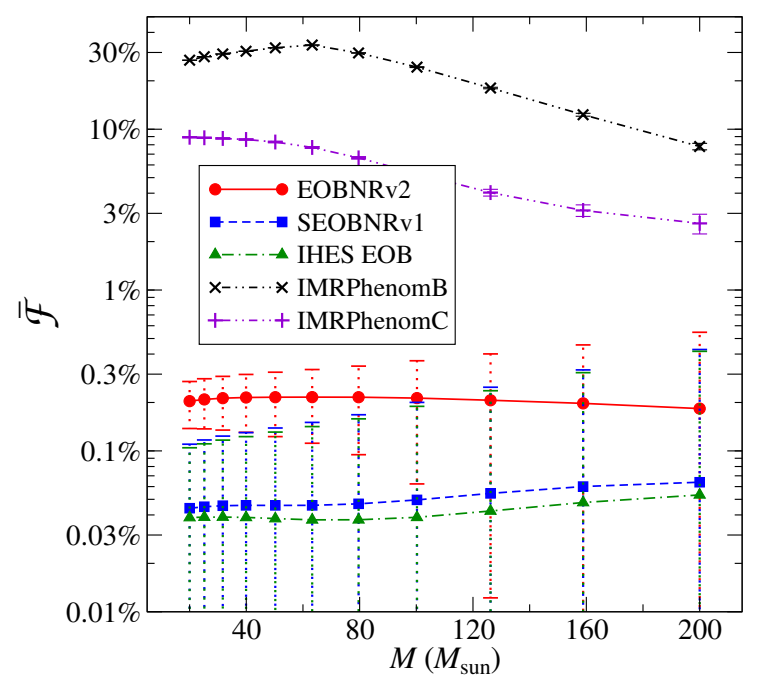

Figure 8. Unfaithfulness of analytical non-spinning $q=10$ waveforms generated by IMRPhenomB, IMRPhenomC and three versions of EOB models with the numerical non-spinning $q=10$ waveform (Case 9 (R10)). The numerical error bars are the same for all sets of results.

that these conclusions for the precessing NRAR waveforms refer only to the $(2,2)$ mode and only to an optimally-oriented precessing system. However, because the numerical waveforms are mildly precessing, we do not expect that these conclusions will change dramatically for a more general orientation.

In summary, for the EOB-spinning comparisons, the non-precessing SEOBNRv1 model performs quite well outside the parameter range in which it was originally calibrated and is also able to match mildly precessing waveforms well. It is certainly effectual in matching all the numerical waveforms of table 1. The results in figure 7 and the discussion above also suggest that the faithfulness of the EOB model can be improved in the high mass-ratio, large asymmetric spins configurations by recalibrating the SEOBNRv1 model using the numerical waveforms produced in this paper, primarily Cases 4 and 8 . Moreover, the 5 precessing numerical waveforms produced in this paper can be used in the calibration of precessing EOB models.

In figure 8 we focus on the largest mass-ratio numerical simulation of table 1 (Case 9 (R10)), which we compare with two non-spinning EOB models, since it is non-spinning. We use the non-spinning limit of the SEOBNRv1 model of 87, 109, which we have discussed above; the EOBNRv2 model developed in [86], which was calibrated to five non-spinning numerical waveforms with mass ratios 1, 2, 3, 4 and 6 from [66], and the recent IHES-EOB model of [88], which was also calibrated to the numerical simulations of [66], but incorporates the most recent, analytical-modeling results from the literature $[222[228]$ that were not available at the time the EOBNRv2 model was developed.

Case 9 (R10) is quite interesting because it is the largest mass-ratio configuration, $q=10$, with sufficient length and accuracy suitable for waveform modeling, and thus it provides an important test of the accuracy of existing EOB models that were calibrated to comparable-mass numerical simulations of $q \leq 6[8688$ and large massratio simulations of $q \geq 1000$ [109, 228]. As we can see in figure 8, for all three EOB 

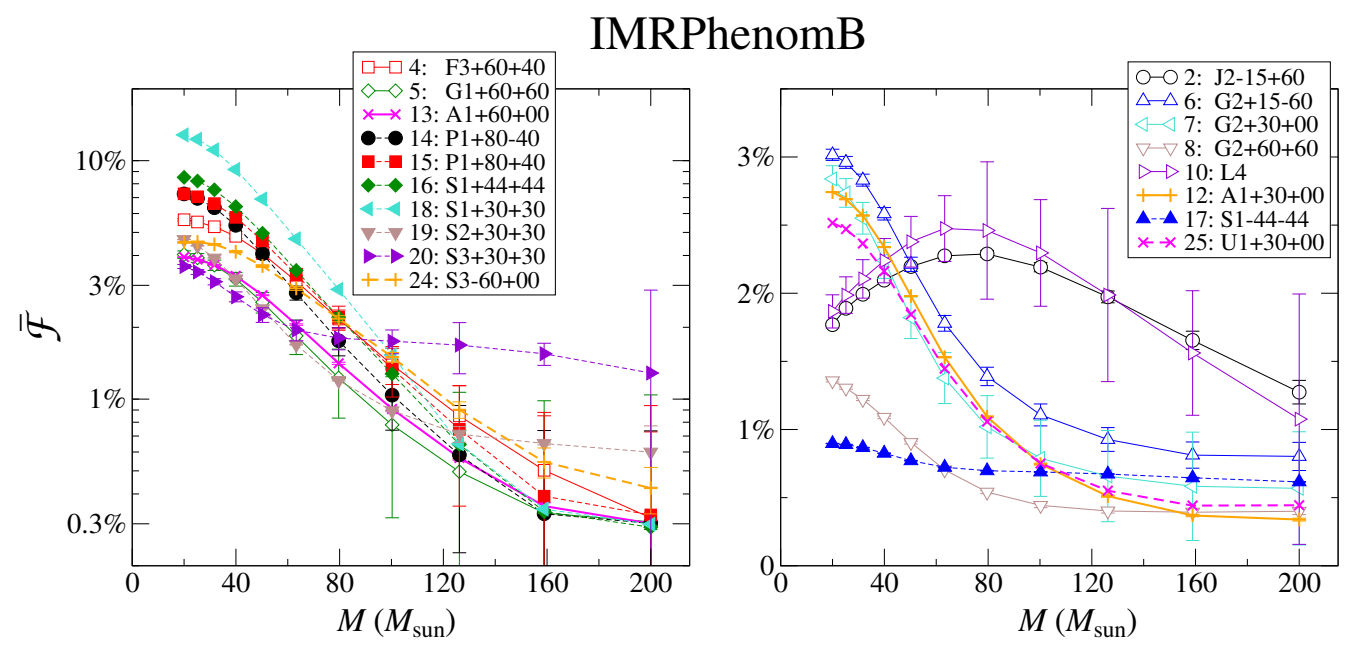

Figure 9. Unfaithfulness $\overline{\mathcal{F}}$ of the IMRPhenomB waveform model compared to the numerical simulations. The horizontal axis shows the total mass, the vertical axis $\overline{\mathcal{F}}$ in percent. The data is split into two panels for readability. As in figure 7 the overlaps for $M \lesssim 100 M_{\odot}$ are affected by the truncation of the overlap integral at the starting frequency of the numerical waveforms. Numerical relativity errorestimates $\delta \overline{\mathcal{F}}$ on the computed unfaithfulness values are plotted, except for Cases 18 and 25 for which $\delta \overline{\mathcal{F}}>20 \%$.

models, the unfaithfulness is a fraction of a percent. We have found that the somewhat larger unfaithfulness of the EOBNRv2 waveform $\left(\sim 10^{-3}\right)$ is due to the fact that in the EOBNRv2 model the peak of $\left|h_{22}\right|$ is enforced to be at the same time as the peak in the orbital frequency. By contrast, in the most recent SEOBNRv1 model, which includes insights from the large mass-ratio limit [224, the peak of $\left|h_{22}\right|$ is chosen to occur $2.5 \mathrm{M}$ earlier than the peak in the orbital frequency. Nevertheless, all unfaithfulness is comparable to or less than the numerical error. Thus, we conclude that all the nonspinning EOB models perform very well, being consistent with the $q=10$ non-spinning numerical simulation. However, longer and more accurate numerical simulations are needed to improve the EOB modeling for mass ratios $q \geq 10$.

\subsection{Results using phenomenological waveform models}

In Figs. 9 and 10, we show the unfaithfulness of the analytical IMRPhenomB and IMRPhenomC waveform models by comparing them with the numerical-relativity waveforms described earlier and included in figure 7.

The IMRPhenomB 91] and IMRPhenomC 92 models are phenomenological inspiral-merger-ringdown waveform families for non-precessing spin binaries. Both models are constructed in the frequency domain with somewhat different ingredients in the low frequency part. The waveforms are parametrized by the total mass $M$, symmetric mass ratio $m_{1} m_{2} /\left(m_{1}+m_{2}\right)^{2}$ and an effective spin parameter $\chi \equiv$ $\left(m_{1} \chi_{1}+m_{2} \chi_{2}\right) / M$. Since the waveforms are described by one spin parameter, these are not meant to be faithful in the case of binaries with unequal spins, and only intended as an effectual template family.

IMRPhenomB is constructed by matching numerical waveforms with adiabatic PN waveforms, notably the 'TaylorT1' approximant, in a matching window. The 
numerical-relativity-PN hybrid waveforms are then parametrized in the frequency domain to get a closed form expression. The IMRPhenomC amplitude is constructed from a PN inspiral amplitude and a ringdown portion, both of which are fit to the model hybrids. IMRPhenomC uses the complete TaylorF2 [12 $999|229| 230]$ PN inspiral phasing. Only the late inspiral and merger phases are fitted in a narrow frequency range $\left[0.1 f_{\mathrm{RD}}, f_{\mathrm{RD}}\right]$ to numerical simulations (where $f_{\mathrm{RD}}$ is the ringdown frequency), while the ringdown waveform is obtained from analytically derived quasi-normal mode expressions for the frequency and attached continuously to the merger phase.

To be consistent with the unfaithfulness calculations shown in Sec. 6.1 for SEOBNRv1, we have computed the unfaithfulness for the phenomenological models as follows. While IMRPhenomB and IMRPhenomC are naturally defined in the Fourier domain as a power series in the frequency $f$, we have chosen to inverse discrete Fourier transform (DFT) them so that the analysis starts out in the time domain. The numerical-relativity and the model waveforms are aligned at an average frequency which is computed over an interval of width $300 M$ starting immediately after the junk radiation has passed. As was done for the SEOBNRv1 unfaithfulness calculation, we apply Planck tapering windows (of width $200 M$ and $20 M$, respectively) to the beginning and end of both timeseries. In addition, the waveforms are further padded with zeros before computing the DFT to increase the frequency resolution. The lowfrequency cutoff for the unfaithfulness integral is chosen as the average frequency defined above, which is close to the starting frequency of each numerical waveform. The same caveats concerning the interpretation of these unfaithfulness results at low masses discussed at the beginning of Sec.6 6 apply here. In addition, we should note that calculating the unfaithfulness directly with the frequency-domain representation of the phenomenological models leads in general to slightly different results. In the approach pursued here, both the numerical-relativity and the model waveforms suffer from similar artefacts due to the DFT near the initial frequency (and the phenomenological waveform further exhibits errors due to the initial inverse DFT bringing it to the time domain), while the amplitude and phase of the phenomenological waveforms vary smoothly with frequency if the frequency-domain representation is used.

The IMRPhenomB and IMRPhenomC models have been calibrated to waveforms of mass ratio $q \leq 4$. Therefore, it is not surprising that both models do not perform very well for the $q=10$ waveform (Case 9 (R10)), as shown in figure 8 However, note that for total mass $\sim 200 M_{\odot}$, the IMRPhenomC waveform has an unfaithfulness of $3 \%$. All models have an ineffectualness of $1 \%$ for binary black holes of mass $100-200 M_{\odot}$, causing a loss of event rate of $3 \%$ if used in advanced LIGO and Virgo searches.

\subsection{Summary of comparison results with analytical models}

We find reasonable agreement between the numerical waveforms produced in this paper and analytical waveforms generated by the EOBNRv2, IHES-EOB, SEOBNRv1, IMRPhenomB and IMRPhenomC models. Since the analytical models were not calibrated using any of the numerical waveforms produced by the NRAR collaboration, these results substantiate the ideas and procedures used in developing the above analytical models.

More quantitatively, the EOBNRv2, IHES-EOB, SEOBNRv1 waveforms agree quite well with the numerical waveforms except for Cases 4 and 8, where artificial features in the SEOBNRv1 waveforms around merger cause large unfaithfulness. 

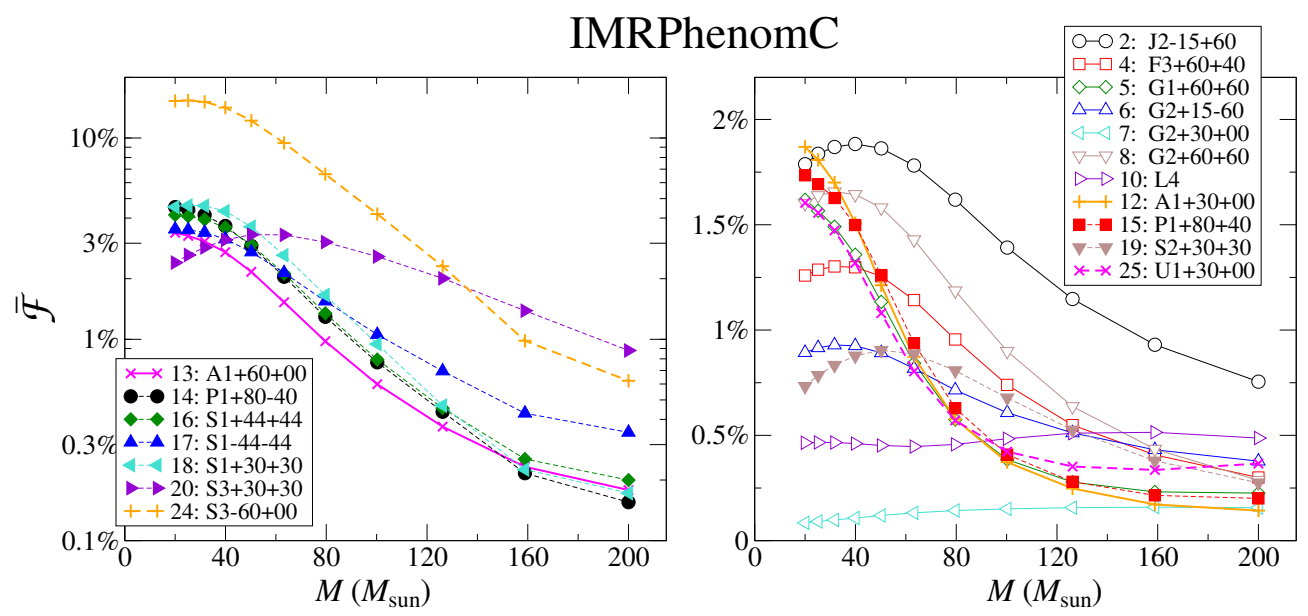

Figure 10. Unfaithfulness $\overline{\mathcal{F}}$ of the IMRPhenomC waveforms compared to the numerical simulations as functions of the total mass. The left panel shows the cases with $\max \overline{\mathcal{F}}>0.02$, the right panel those with $\max \overline{\mathcal{F}}<0.02$. As in figure 7 the overlaps for $M \lesssim 100 M_{\odot}$ are affected by the truncation of the overlap integral at the starting frequency of the numerical waveforms.

Moreover, SEOBNRv1 waveforms are faithful toward both low and high total masses and the unfaithfulness shows less dependence on the total mass, indicating a consistent agreement during the inspiral, plunge, merger and ringodwn stages across the entire frequency range. We estimated that EOB models have ineffectualness below $1 \%$ against all the non-precessing numerical waveforms produced in this paper for binary black holes with mass $100-200 M_{\odot}$, for which the numerical-relativity waveforms fully span the advanced LIGO bandwidth.

The IMRPhenomB and IMRPhenomC waveforms agree quite well with numerical waveforms at high total masses and can be quite faithful when $M>100 M_{\odot}$. However, the agreement is less satisfactory at low total masses, but this is not so surprising because those waveforms use a single, effective spin and were developed as effectual templates. The unfaithfulness of most IMRPhenomB waveforms increases toward lower total mass (with negative slope) because the phasing coefficients in the IMRPhenomB model do not reduce to the PN ones, but are obtained through a fit of the hybrid waveform. Using the TaylorF2 PN phasing, the IMRPhenomC waveforms show improved agreement with numerical waveforms at low total masses. We estimated that phenomenological models have ineffectualness below $1 \%$ against all the non-precessing numerical waveforms produced here for binary black holes with mass $100-200 M_{\odot}$.

\section{Conclusions}

The Numerical-Relativity-Analytical-Relativity (NRAR) Collaboration has the goals of producing numerical waveforms from compact binaries and using them to construct and validate analytical waveform models to search for binary black holes with gravitational-wave detectors. In this first stage of the project, 22 new waveforms were computed and rigorously analysed together with 3 contributed waveforms. The new waveforms were of higher quality than most of the previously published waveforms. 
Furthermore, a larger region of parameter space was covered than before, including several unequal-mass, unequal-spin binaries with aligned spins, and several precessing runs to begin exploration of waveform models for precessing configurations.

For the first time, we compared analytical models previously calibrated to numerical waveforms to newly produced waveforms. We found that the spinning, non-precessing EOB model (SEOBNRv1) 87], which was previously calibrated to only 2 equal-mass, equal-spin, non-precessing numerical waveforms and 5 nonspinning waveforms with mass ratios $q=1,2,3,4,6$ has ineffectualness below $1 \%$ in matching all the non-precessing numerical waveforms when the binary's total mass is $\sim 100-200 M_{\odot}$. Moreover, the non-spinning EOB models 86 88. (EOBNRv2, SEOBNRv1, IHES-EOB) previously calibrated only to the 5 non-spinning waveforms with mass ratios $q=1,2,3,4,6$ have unfaithfulness below $0.3 \%$ in matching the numerical waveform with mass ratio $q=10$. We recall that an ineffectualness of $1 \%$ causes a loss of event rates of $\sim 3 \%$. Quite interestingly, we found that the EOB unfaithfulness and ineffectualness is small towards both low and high total masses (except for two cases where a non-monotonicity of the frequency evolution close to merger causes larger unfaithfulness). This nice performance indicates a consistent agreement between EOB and numerical waveforms during the inspiral, plunge, merger and ringdown stages. These results imply that the EOB approach of building analytical templates is quite robust (for both detection and parameter estimation) in interpolating numerical waveforms away from the points in the parameter space where they were employed to calibrate the EOB templates. This success relies also on the fact that the EOB model incorporates accurate results from PN theory and perturbation theory, notably the Regge-Wheeler-equation and Teukolsky-equation results 109 228.

We found that the phenomenological models 91, 92 (IMRPhenomB and IMPRPhenomC), which were previously calibrated to 25 spinning, non-precessing waveforms with mild spins and mass ratios $q \leq 4$, have somewhat larger unfaithfulness, especially toward low total masses. However, this is not surprising, since those models use a single, effective spin and were conceived as effectual templates. More importantly, their ineffectualness is below $1 \%$ against all the non-precessing numerical waveforms produced in this paper for binary black holes with mass $100-200 M_{\odot}$, for which the numerical-relativity waveforms fully span the advanced LIGO bandwidth. The only exception is the ineffectualness of IMRPhenomB against the $q=10$ waveform at high masses. However, this problem does not exist in the later, enhanced IMRPhenomC model. The faithfulness of EOB and phenomenological models can be improved in the future by re-calibrating those models to the NRAR waveforms. We also identified a few shortcomings in both classes of waveform family, and expect that these can be rectified in future analytic models, using the numerical-relativity waveforms presented here.

The simulations performed pushed all participating NR groups into unknown territory, along one or more of these dimensions:

(i) Simulations of more extreme parameters, for instance very high mass-ratios up to $q=10$ - and performing simulations with both non-zero spin and unequal masses.

(ii) Longer and more accurate simulations than before (20 usable gravitational-wave cycles before merger, with cumulative phase-error $\leq 0.25$ radians and amplitude error of $\leq 1 \%$ ).

(iii) Determining initial data parameters resulting in low eccentricity, $e \lesssim 2 \times 10^{-3}$. 
(iv) Automating codes to increase the number of simulations that can be performed with the available human resources.

(v) Exhaustive cross-comparisons, and the resultant error checking and searching for the origins of discrepancies.

In line with expectations, the accurate simulation of systems with high spins, high mass ratios and (for finite difference codes) large numbers of orbits proved challenging, requiring significant computational resources. Gravitational-wave extraction required significant attention. To push errors into the accuracy regime chosen for this project, extraction must be performed at distances of $r>100 M$, or a more advanced extraction method such as CCE must be employed. Moreover, gravitational-wave extrapolation to infinite extraction radius (for codes that do not extract the waveforms at future null infinity) is essential to achieve the accuracy goals. Errors at finite extraction radius decay so slowly with increased extraction radius that it is impractical to achieve higher gravitational-wave accuracy by simply increasing the extraction radius. Moreover, reliable extrapolation requires several $(\gtrsim 5)$ extraction radii which are spaced over a significant range of radii; the ratio of outermost extraction radius to innermost extraction radius should be $\gtrsim 3$. Furthermore, this project saw the first attempt to combine gravitational-wave extrapolation with fixed-frequency integration to compute $h$ from $\Psi_{4}$. Unfortunately, robust error estimates are difficult to obtain. Future work should address this.

Following on from the NINJA project, this project represents one of the first attempts at large-scale collaboration of this type in this field, and it is only now becoming clear what aspects of data management need to be improved and developed as best-practices. The size of the data set being computed made it difficult to collect, distribute and analyze the data across three continents. Version control of the data and metadata proved essential. While the Subversion version-control system might be adequate for the metadata, it proved deficient to handle a data set of about 150 GByte. At the very least, metadata needs to be provided independently of the main data set, to allow users to download only the small metadata files for preliminary consistency checks. A significant improvement in data-management process for numerical relativity would result if metadata submissions were automatically checked and validated, allowing the submitter to see immediately the problems with the submission, rather than waiting for these quantities to be needed by the analysis, and introducing a turnaround time on the order of days in each case.

The NRAR collaboration now has the ability to take numerical-relativity waveforms in a standard format [199] and automatically process them into a form suitable for comparison with analytic models. While certain consistency checks are applied automatically, this 'pipeline' can still not be used blindly, as there are frequently complications which arise from dealing with a diverse set of waveforms with varying characteristics. However, the existence of automated tools makes it possible in principle to greatly expand the number of waveforms which can be analysed as they become available. Given how surprisingly hard this project turned out to be, one should be mindful that further improvements to numerical simulations might also be hard, e.g. doubling the time span, halving errors, or going to untested regions of parameter space. While numerical relativity has made tremendous progress over the last years, pushing beyond the current limits of simulations is still a research project where unpredictable new problems can and do arise. 
We note that during the last stages of completion of this manuscript two papers appeared on arXiv reporting the production of hundreds of new numerical-relativity waveforms [231, 232. The two papers were written by two of the NR groups that are part of the NRAR collaboration. When planning the next steps of the NRAR collaboration, these results will be taken into account to avoid duplication of numerical simulations.

\section{Acknowledgments}

We thank Beverly Berger and Kip Thorne for helping to establish the NRAR collaboration, Duncan Brown and Frans Pretorius for their work as members of the NRAR executive committee, Duncan Brown, Evan Ochsner and B. Sathyaprakash for useful discussions in the first stage of the NRAR collaboration when the scientific plan and accuracy requirements were established. We also thank John Baker, Peter Diener, Bernard Kelly and Riccardo Sturani for useful interactions. It is also a pleasure to thank David Hilditch for useful discussions on the hyperbolicity of BSSN, Roman Gold for useful discussions on requirements for accurate computation of gravitational waves and P. Ajith for help with performing and interpreting the IMRPhenom comparisons. We also thank Bruce Loftis and Christal Yost at the National Institute for Computational Sciences (NICS) for their support.

Caltech acknowledges support from the Sherman Fairchild Foundation and NSF grants PHY-1068881, PHY-1005655, and DMS-1065438. M. Hannam was supported by Science and Technology Facilities Council grants ST/H008438/1 and ST/I001085/1. CITA acknowledges support from NSERC of Canada, the Canada Chairs Program, and the Canadian Institute for Advanced Research. Cornell acknowledges support from the Sherman Fairchild Foundation and NSF grants PHY-0969111 and PHY1005426. FAU acknowledges financial support via the NSF grants 0855315 and 1204334. GA Tech acknowledges financial support via NSF grants 1205864, 1212433, 0903973, 0941417, and 0955825. Jena and the AEI acknowledge financial support via the DFG SFB/Transregio 7. U. Sperhake acknowledges financial support via the Ramón y Cajal Programme of the Ministry of Education and Science in Spain, the FP7-PEOPLE-2011-CIG grant 293412 CBHEO, the STFC GR Roller grant ST/I002006/1, the ERC Starting grant ERC-2010-StG DyBHo, and the FP7PEOPLE-2011-IRSES grant 295189 NRHEP. A. Nerozzi acknowledges support by the Fundação para a Ciência e Tecnologia through grants SFRH/BPD/47955/2008 and PTDC/FIS/116625/2010. H. Witek acknowledges support by the $E R C$ 2011-StG 279363-HiDGR ERC Starting Grant and by the DyBHo-256667 ERC Starting Grant. H.W. acknowledges support by FCT-Portugal through grant nos. SFRH/BD/46061/2008 and CERN/FP/123593/2011 at early stages of this work. C. Reisswig acknowledges financial support from NASA via the Einstein Postdoctoral Fellowship grant PF2-130099 awarded by the Chandra X-ray center, which is operated by the Smithsonian Astrophysical Observatory for NASA under contract NAS8-03060. RIT acknowledges financial support via NSF grants AST-1028087, PHY-0929114, PHY-0969855, PHY-0903782, OCI-0832606, and DRL-1136221, and NASA grant 07ATFP07-0158. H. Nakano would also like to acknowledge support by the Grandin-Aid for Scientific Research (24103006). UIUC acknowledges financial support via NSF grants PHY-0963136, AST-1002667, and NASA grants NNX11AE11G, NNX13AH44G. V. Paschalidis would also like to acknowledge financial support from a Fortner Research Fellowship. UMD acknowledges financial support via 
NSF grants PHY-0903631 and PHY-1208881, and NASA grants NNX09AI81G and NNX12AN10G.

We also acknowledge the hospitality of the Kavli Institute for Theoretical Physics during the program "Chirps, Mergers and Explosions" (supported by the NSF grant PHY11-25915).

Computing time for this project was provided by the NSF on the TeraGrid (now XSEDE) systems Athena and Kraken at NICS. AEI used computational resources on the Datura cluster at AEI. Caltech and Cornell used additional computational resources on the Zwicky cluster at Caltech, which is supported by the Sherman Fairchild Foundation and by NSF award PHY-0960291, and on the NSF XSEDE network under grant TG-PHY990007N. CITA used additional computational resources at the SciNet HPC Consortium on the GPC system. FAU used additional computational resources via TeraGrid allocations TG-AST100021 and TG-PHY100051 at NICS on the Kraken system. GA Tech used additional computational resources via XSEDE allocation TG-PHY120016, and at Georgia Tech on the Cygnus cluster. JCP used additional computational resources at the LRZ, Munich, and as part of the European PRACE petascale computing initiative on the clusters Hermit, Curie and SuperMUC; simulations were were also carried out at Advanced Research Computing (ARCCA) at Cardiff. US, AN and HW used additional computational resources via XSEDE allocation PHY-090003 at SDCS on the Trestles system and at NICS on the Kraken system, via the Barcelona Supercomputing Center (BSC) allocation AECT-2012-3-0011, via the Centro de Supercomputación de Galicia (CESGA) allocation ICTS-2011-234, the Baltasar-Sete-Sóis cluster at CENTRA/IST Lisbon, the CANE cluster in Poland through PRACE DECI-7 and at the DiRAC HPC Facility on the COSMOS supercomputer supported by STFC and BIS. RIT used additional computational resources at TACC on the Ranger system via XSEDE allocation TG-PHY060027N, and at RIT on the NewHorizons/BlueSky system supported by NSF grants PHY-0722703, DMS-0820923, AST-1028087 and PHY-1229173. UIUC used additional computational resources at TACC on the Stampede system via XSEDE allocation TG-MCA99S008. Hosting for the NRAR data set and collaboration tools was provided by the Syracuse University Gravitationalwave Group, supported by NSF awards PHY-0847611, PHY-1040231 and PHY1104371.

The EOBNRv2, SEOBNRv1, IMRPhenomB and IMRPhenomC templates were produced using the public LIGO Algorithm Library [233, while the IHES-EOB templates were produced using the public code in 234.

\section{References}

[1] Abbott B P et al. (LIGO Scientific Collaboration) 2009 Rep. Prog. Phys. 72076901 (arXiv 0711.3041

[2] Accadia T et al. (Virgo Collaboration) 2012 J. Instrum. 73012

[3] Grote H (for the LIGO Scientific Collaboration) 2010 Class. Quantum Grav. 27084003

[4] Harry G M (for the LIGO Scientific Collaboration) 2010 Class. Quantum Grav. 27084006

[5] Abadie J et al. (LIGO Scientific Collaboration and Virgo Collaboration) 2010 Class. Quantum Grav. 27173001 (arXiv 1003.2480)

[6] Aasi J et al. (LIGO Scientific Collaboration and Virgo Collaboration) 2013 (arXiv 1304.0670)

[7] Somiya K (for the KAGRA Collaboration) 2012 Class. Quantum Grav. 29124007 (arXiv 1111. $7185)$

[8] IndIGO website: http://www.gw-indigo.org/tiki-index.php

[9] Amaro Seoane P et al. (eLISA Consortium) 2013 (arXiv 1305.5720) 
[10] ESA LISA website: http://sci.esa.int/lisa

[11] Dhurandhar S V and Sathyaprakash B S 1994 Phys. Rev. D 491707

[12] Sathyaprakash B S and Dhurandhar S V 1991 Phys. Rev. D 443819

[13] Balasubramanian R, Sathyaprakash B S and Dhurandhar S V 1996 Phys. Rev. D 533033 (arXiv gr-qc/9508011)

[14] Owen B J and Sathyaprakash B S 1999 Phys. Rev. D 60022002 (arXiv gr-qc/9808076

[15] Apostolatos T A 1996 Phys. Rev. D 542421

[16] Allen B, Anderson W G, Brady P R, Brown D A and Creighton J D E 2012 Phys. Rev. D 85 122006 (arXiv gr-qc/0509116)

[17] Finn L S and Chernoff D F 1993 Phys. Rev. D 472198 (arXiv gr-qc/9301003)

[18] Babak S et al. 2013 Phys. Rev. D 87024033 (arXiv 1208.3491)

[19] Babak S, Balasubramanian R, Churches D, Cokelaer T and Sathyaprakash B S 2006 Class. Quantum Grav. 235477 (arXiv gr-qc/0604037)

[20] Blanchet L 2006 Living Rev. Rel. 94 URL http://relativity.livingreviews.org/Articles/ 1rr-2006-4

[21] Abadie J et al. (LIGO Scientific Collaboration and Virgo Collaboration) 2012 Phys. Rev. D 85 082002 (arXiv 1111.7314 )

[22] Blanchet L, Damour T, Iyer B R, Will C M and Wiseman A 1995 Phys. Rev. Lett. 743515 (arXiv $\mathrm{gr}-\mathrm{qc} / 9501027$ )

[23] Damour T, Jaranowski P and Schäfer G 2001 Phys. Lett. B 513147 (arXiv gr-qc/0105038)

[24] Itoh Y and Futamase T 2003 Phys. Rev. D 68121501 (arXiv gr-qc/0310028)

[25] Blanchet L, Damour T, Esposito-Farèse G and Iyer B R 2004 Phys. Rev. Lett. 93091101 (arXiv $\mathrm{gr}-\mathrm{qc} / 0406012$ )

[26] Buonanno A, Iyer B, Ochsner E, Pan Y and Sathyaprakash B S 2009 Phys. Rev. D 80084043 $(\operatorname{arXiv} 0907.0700)$

[27] Brown D A, Kumar P and Nitz A H 2013 Phys. Rev. D 87082004 (arXiv 1211.6184)

[28] Hannam M, Husa S, González J A, Sperhake U and Brügmann B 2008 Phys. Rev. D 77044020 (arXiv 0706.1305

[29] Boyle M, Brown D A, Kidder L E, Mroué A H, Pfeiffer H P, Scheel M A, Cook G B and Teukolsky S A 2007 Phys. Rev. D 76124038 (arXiv 0710.0158)

[30] Hannam M, Husa S, Brügmann B and Gopakumar A 2008 Phys. Rev. D 78104007 (arXiv 0712. 3787)

[31] Boyle M, Buonanno A, Kidder L E, Mroué A H, Pan Y, Pfeiffer H P and Scheel M A 2008 Phys. Rev. D 78104020 (arXiv 0804.4184

[32] Hinder I, Herrmann F, Laguna P and Shoemaker D 2010 Phys. Rev. D 82024033 (arXiv 0806. 1037)

[33] Campanelli M, Lousto C O, Nakano H and Zlochower Y 2009 Phys. Rev. D 79084010 (arXiv:0808.0713)

[34] Hannam M, Husa S, Ohme F, Muller D and Brügmann B 2010 Phys. Rev. D 82124008 (arXiv:1007.4789)

[35] MacDonald I, Mroué A H, Pfeiffer H P, Boyle M, Kidder L E, Scheel M A, Szilágyi B and Taylor N W 2013 Phys. Rev. D 87024009 (arXiv 1210.3007)

[36] Hemberger D A, Lovelace G, Loredo T J, Kidder L E, Scheel M A, Szilágyi B, Taylor N W and Teukolsky S A 2013 Phys. Rev. D 88064014 (arXiv 1305.5991)

[37] Pretorius F 2005 Phys. Rev. Lett. 95121101 (arXiv gr-qc/0507014)

[38] Campanelli M, Lousto C O, Marronetti P and Zlochower Y 2006 Phys. Rev. Lett. 96111101 (arXiv $\mathrm{gr}-\mathrm{qc} / 0511048$ )

[39] Baker J G, Centrella J M, Choi D I, Koppitz M and van Meter J R 2006 Phys. Rev. Lett. 96 111102 (arXiv gr-qc/0511103)

[40] Pretorius F 2009 Binary black hole coalescence Physics of Relativistic Objects in Compact Binaries: from Birth to Coalescence ed Colpi M, Casella P, Gorini V, Moschella U and Possenti A (Heidelberg, Germany: Springer) pp 305-369 (arXiv:0710.1338)

[41] Hannam M 2009 Class. Quantum Grav. 26114001 (arXiv 0901.2931)

[42] Centrella J M, Baker J G, Kelly B J and van Meter J R 2010 Ann. Rev. Nucl. Part. Sci. 60 75 (arXiv 1010.2165)

[43] Hinder I 2010 Class. Quantum Grav. 27114004 (arXiv 1001.5161)

[44] Shibata M and Taniguchi K 2011 Living Rev. Rel. 146 URL http://relativity. livingreviews.org/Articles/lrr-2011-6

[45] Sperhake U, Berti E and Cardoso V 2013 Comptes Rendus Physique 14306 (arXiv 1107.2819)

[46] Pfeiffer H P 2012 Class. Quantum Grav. 29124004 (arXiv 1203.5166)

[47] Tichy W and Marronetti P 2007 Phys. Rev. D 76061502 (arXiv gr-qc/0703075) 
[48] Tichy W and Marronetti P 2008 Phys. Rev. D 78081501 (arXiv 0807.2985)

[49] Schmidt P, Hannam M, Husa S and Ajith P 2011 Phys. Rev. D 84024046 (arXiv 1012.2879)

[50] O'Shaughnessy R, Vaishnav B, Healy J, Meeks Z and Shoemaker D 2011 Phys. Rev. D 84 124002 (arXiv 1109.5224)

[51] Mroué A H and Pfeiffer H P 2012 (arXiv 1210.2958)

[52] O'Shaughnessy R, Healy J, London L, Meeks Z and Shoemaker D 2012 Phys. Rev. D 85084003 (arXiv 1201.2113 )

[53] O'Shaughnessy R, London L, Healy J and Shoemaker D 2013 Phys. Rev. D 87044038 (arXiv 1209.3712)

[54] Pekowsky L, Healy J, Shoemaker D and Laguna P 2013 Phys. Rev. D 87084008 (arXiv 1210. 1891)

[55] Healy J, Laguna P, Pekowsky L and Shoemaker D 2013 Phys. Rev. D 88024034 (arXiv 1302. 6953)

[56] Lovelace G, Owen R, Pfeiffer H P and Chu T 2008 Phys. Rev. D 78084017 (arXiv 0805.4192)

[57] Lovelace G, Scheel M A and Szilágyi B 2011 Phys. Rev. D 83024010 (arXiv 1010.2777)

[58] Lovelace G, Boyle M, Scheel M A and Szilágyi B 2012 Class. Quantum Grav. 29045003 (arXiv 1110.2229$)$

[59] Lousto C O, Nakano H, Zlochower Y, Mundim B C and Campanelli M 2012 Phys. Rev. D 85 124013 (arXiv 1203.3223)

[60] Lousto C O and Zlochower Y 2011 Phys. Rev. Lett. 106041101 (arXiv 1009.0292)

[61] Sperhake U, Cardoso V, Ott C D, Schnetter E and Witek H 2011 Phys. Rev. D 84084038 (arXiv:1105.5391)

[62] Lousto C O and Zlochower Y 2013 Phys. Rev. D 88024001 (arXiv 1304.3937)

[63] Campanelli M, Lousto C O, Zlochower Y and Merritt D 2007 Astrophys. J. 659 L5 $(\operatorname{arXiv} \mathrm{gr-qc/0701164)}$

[64] González J A, Hannam M D, Sperhake U, Brügmann B and Husa S 2007 Phys. Rev. Lett. 98 231101 (arXiv gr-qc/0702052)

[65] Lousto C O and Zlochower Y 2011 Phys. Rev. Lett. 107231102 (arXiv 1108.2009)

[66] Buchman L T, Pfeiffer H P, Scheel M A and Szilágyi B 2012 Phys. Rev. D 86084033 (arXiv: 1206.3015)

[67] Healy J, Bode T, Haas R, Pazos E, Laguna P, Shoemaker D M and Yunes N 2012 Class. Quantum Grav. 29232002 (arXiv 1112.3928)

[68] Barausse E, Palenzuela C, Ponce M and Lehner L 2013 Phys. Rev. D 87081506 (arXiv 1212. 5053)

[69] Berti E, Cardoso V, Gualtieri L, Horbatsch M and Sperhake U 2013 Phys. Rev. D 87124020 (arXiv 1304.2836

[70] Cao Z, Galaviz P and Li L F 2013 Phys. Rev. D 87104029

[71] Buonanno A and Damour T 1999 Phys. Rev. D 59084006 (arXiv gr-qc/9811091)

[72] Buonanno A and Damour T 2000 Phys. Rev. D 62064015 (arXiv gr-qc/0001013)

[73] Damour T, Jaranowski P and Schäfer G 2000 Phys. Rev. D 62084011 (arXiv gr-qc/0005034)

[74] Davis M, Ruffini R and Tiomno J 1972 Phys. Rev. D 52932

[75] Goebel C J 1972 Astrophys. J. Lett. 172 L95

[76] Mashhoon B 1985 Phys. Rev. D 31290

[77] Price R H and Pullin J 1994 Phys. Rev. Lett. 723297 (arXiv gr-qc/9402039)

[78] Buonanno A, Chen Y and Damour T 2006 Phys. Rev. D 74104005 (arXiv gr-qc/0508067)

[79] Buonanno A, Cook G B and Pretorius F 2007 Phys. Rev. D 75124018 (arXiv gr-qc/0610122)

[80] Buonanno A, Pan Y, Baker J G, Centrella J, Kelly B J, McWilliams S T and van Meter J R 2007 Phys. Rev. D 76104049 (arXiv 0706.3732)

[81] Damour T, Nagar A, Dorband E N, Pollney D and Rezzolla L 2008 Phys. Rev. D 77084017 (arXiv 0712.3003)

[82] Damour T, Nagar A, Hannam M, Husa S and Brügmann B 2008 Phys. Rev. D 78044039 (arXiv 0803.3162)

[83] Buonanno A, Pan Y, Pfeiffer H P, Scheel M A, Buchman L T and Kidder L E 2009 Phys. Rev. D 79124028 (arXiv 0902.0790)

[84] Damour T and Nagar A 2009 Phys. Rev. D 79081503 (arXiv 0902.0136)

[85] Pan Y, Buonanno A, Buchman L T, Chu T, Kidder L E, Pfeiffer H P and Scheel M A 2010 Phys. Rev. D 81084041 (arXiv 0912.3466)

[86] Pan Y, Buonanno A, Boyle M, Buchman L T, Kidder L E, Pfeiffer H P and Scheel M A 2011 Phys. Rev. D $84124052(\operatorname{arXiv} 1106.1021)$

[87] Taracchini A, Pan Y, Buonanno A, Barausse E, Boyle M, Chu T, Lovelace G, Pfeiffer H P and Scheel M A 2012 Phys. Rev. D 86024011 (arXiv 1202.0790) 
[88] Damour T, Nagar A and Bernuzzi S 2013 Phys. Rev. D 87084035 (arXiv 1212.4357)

[89] Pan Y, Buonanno A, Baker J G, Centrella J, Kelly B J, McWilliams S T, Pretorius F and van Meter J R 2008 Phys. Rev. D 77024014 (arXiv 0704.1964)

[90] Ajith P et al. 2008 Phys. Rev. D 77104017 (arXiv 0710.2335)

[91] Ajith P et al. 2011 Phys. Rev. Lett. 106241101 (arXiv 0909.2867)

[92] Santamaría L et al. 2010 Phys. Rev. D 82064016 (arXiv 1005.3306)

[93] Abadie J et al. (LIGO Scientific Collaboration and Virgo Collaboration) 2011 Phys. Rev. D 83 122005 (arXiv: 1102.3781)

[94] Aasi J et al. (LIGO Scientific Collaboration and Virgo Collaboration) 2013 Phys. Rev. D 87 022002 (arXiv: 1209.6533)

[95] Abadie J et al. (LIGO Scientific Collaboration and Virgo Collaboration) 2012 Phys. Rev. D 85 102004 (arXiv 1201.5999 )

[96] Aasi J et al. (LIGO Scientific Collaboration and Virgo Collaboration) 2013 Phys. Rev. D 88 062001 (arXiv: 1304.1775)

[97] Aylott B et al. 2009 Class. Quantum Grav. 26165008 (arXiv 0901.4399)

[98] Cadonati L et al. 2009 Class. Quantum Grav. 26114008 (arXiv:0905.4227)

[99] Ajith P et al. 2012 Class. Quantum Grav. 29124001 (arXiv 1201.5319)

[100] Hannam M et al. 2009 Phys. Rev. D 79084025 (arXiv 0901.2437)

[101] Baker J G, Campanelli M, Pretorius F and Zlochower Y 2007 Class. Quantum Grav. 24 S25 (arXiv $\mathrm{gr}-\mathrm{qc} / 0701016)$

[102] Shoemaker D (LIGO Scientific Collaboration) 2010 Advanced LIGO anticipated sensitivity curves LIGO Document T0900288-v3 URL https://dcc.ligo.org/cgi-bin/DocDB/ ShowDocument?docid=2974

[103] Scheel M A, Boyle M, Chu T, Kidder L E, Matthews K D and Pfeiffer H P 2009 Phys. Rev. D 79024003 (arXiv 0810.1767)

[104] Ajith P 2008 Class. Quantum Grav. 25114033 (arXiv 0712.0343

[105] Buonanno A, Chen Y, Pan Y and Vallisneri M 2004 Phys. Rev. D 70104003 (arXiv gr-qc/ 0405090)

[106] Apostolatos T A, Cutler C, Sussman G J and Thorne K S 1994 Phys. Rev. D 496274

[107] Pan Y, Buonanno A, Chen Y and Vallisneri M 2004 Phys. Rev. D 69104017 (arXivgr-qc/ 0310034

[108] Ajith P 2011 Phys. Rev. D 84084037 (arXiv 1107.1267)

[109] Barausse E, Buonanno A, Hughes S A, Khanna G, O'Sullivan S and Pan Y 2012 Phys. Rev. D $85024046(\operatorname{arXiv} 1110.3081)$

[110] Pürrer M, Hannam M, Ajith P and Husa S 2013 Phys. Rev. D 88064007 (arXiv 1306.2320)

[111] Boyle L, Kesden M and Nissanke S 2008 Phys. Rev. Lett. 100151101 (arXiv 0709.0299)

[112] Buonanno A, Kidder L E and Lehner L 2008 Phys. Rev. D 77026004 (arXiv 0709.3839)

[113] Rezzolla L, Barausse E, Dorband E N, Pollney D, Reisswig C, Seiler J and Husa S 2008 Phys. Rev. D 78044002 (arXiv 0712.3541)

[114] Barausse E and Rezzolla L 2009 Astrophys. J. Lett. 704 L40 (arXiv 0904.2577)

[115] Barausse E, Morozova V and Rezzolla L 2012 Astrophys. J. 75863 (arXiv: 1206.3803)

[116] Cannon K, Chapman A, Hanna C, Keppel D, Searle A C and Weinstein A J 2010 Phys. Rev. D $82044025(\operatorname{arXiv} 1005.0012$

[117] Cannon K, Hanna C and Keppel D 2011 Phys. Rev. D 84084003 (arXiv 1101.4939)

[118] Cannon K, Hanna C and Keppel D 2012 Phys. Rev. D 85081504 (arXiv 1108.5618)

[119] Field S E, Galley C R, Herrmann F, Hesthaven J S, Ochsner E and Tiglio M 2011 Phys. Rev. Lett. 106221102 (arXiv 1101.3765 )

[120] Caudill S, Field S E, Galley C R, Herrmann F and Tiglio M 2012 Class. Quantum Grav. 29 095016 (arXiv 1109.5642 )

[121] Field S E, Galley C R and Ochsner E 2012 Phys. Rev. D 86084046 (arXiv 1205.6009)

[122] Miller M A 2005 Phys. Rev. D 71104016 (arXiv gr-qc/0502087)

[123] Lindblom L, Owen B J and Brown D A 2008 Phys. Rev. D 78124020 (arXiv 0809.3844)

[124] Lindblom L 2009 Phys. Rev. D 80042005 (arXiv 0906.5153)

[125] Lindblom L 2009 Phys. Rev. D 80064019 (arXiv 0907.0457)

[126] Lindblom L, Baker J G and Owen B J 2010 Phys. Rev. D 82 084020 (arXiv 1008.1803

[127] Damour T, Nagar A and Trias M 2011 Phys. Rev. D 83024006 (arXiv 1009.5998)

[128] Hannam M, Husa S, Ohme F and Ajith P 2010 Phys. Rev. D 82124052 (arXiv: 1008.2961)

[129] Boyle M 2011 Phys. Rev. D 84064013 (arXiv 1103.5088)

[130] Ohme F, Hannam M and Husa S 2011 Phys. Rev. D 84064029 (arXiv 1107.0996)

[131] MacDonald I, Nissanke S and Pfeiffer H P 2011 Class. Quantum Grav. 28134002 (arXiv 1102. 5128) 
[132] Littenberg T B, Baker J G, Buonanno A and Kelly B J 2012 Phys. Rev. D 87104003 (arXiv 1210.0893$)$

[133] Poisson E and Will C M 1995 Phys. Rev. D 52848 (arXiv gr-qc/9502040)

[134] Baird E, Fairhurst S, Hannam M and Murphy P 2013 Phys. Rev. D 87024035 (arXiv 1211. 0546)

[135] Hannam M, Brown D A, Fairhurst S, Fryer C L and Harry I W 2013 Astrophys. J. 766 L14 (arXiv 1301.5616$)$

[136] Winicour J 2012 Living Rev. Rel. 152 URL http://relativity.livingreviews.org/ Articles/lrr-2012-2

[137] Friedrich H 1985 Commun. Math. Phys. 100525

[138] Garfinkle D 2002 Phys. Rev. D 65044029 (arXiv gr-qc/0110013)

[139] Pretorius F 2005 Class. Quantum Grav. 22425 (arXiv/gr-qc/0407110

[140] Shibata M and Nakamura T 1995 Phys. Rev. D 525428

[141] Baumgarte T W and Shapiro S L 1999 Phys. Rev. D 59024007 (arXiv gr-qc/9810065)

[142] Hannam M, Husa S, Pollney D, Brügmann B and Ó Murchadha N 2007 Phys. Rev. Lett. 99 241102 (arXiv $\mathrm{gr}-\mathrm{qc} / 0606099)$

[143] Hannam M, Husa S, Ohme F, Brügmann B and Ó Murchadha N 2008 Phys. Rev. D 78064020 (arXiv:0804.0628)

[144] Brown J D 2009 Phys. Rev. D 80084042 (arXiv 0908.3814)

[145] Spectral Einstein Code website: http://www.black-holes.org/SpEC.html

[146] Lindblom L, Scheel M A, Kidder L E, Owen R and Rinne O 2006 Class. Quantum Grav. 23 S447-S462 (arXiv gr-qc/0512093)

[147] Lindblom L and Szilágyi B 2009 Phys. Rev. D 80084019 (arXiv 0904.4873)

[148] Szilágyi B, Lindblom L and Scheel M A 2009 Phys. Rev. D 80124010 (arXiv 0909.3557)

[149] Caudill M, Cook G B, Grigsby J D and Pfeiffer H P 2006 Phys. Rev. D 74064011 (arXiv gr-qc/0605053)

[150] Cook G B and Pfeiffer H P 2004 Phys. Rev. D 70104016 (arXiv gr-qc/0407078)

[151] Buonanno A, Kidder L E, Mroué A H, Pfeiffer H P and Taracchini A 2011 Phys. Rev. D 83 104034 (arXiv 1012.1549 )

[152] Ossokine S, Kidder L E and Pfeiffer H P 2013 Phys. Rev. D 88084031 (arXiv 1304.3067

[153] Scheel M A, Pfeiffer H P, Lindblom L, Kidder L E, Rinne O and Teukolsky S A 2006 Phys. Rev. D 74104006 (arXiv gr-qc/0607056)

[154] Chu T, Pfeiffer H P and Scheel M A 2009 Phys. Rev. D 80124051 (arXiv:0909.1313)

[155] Hemberger D A, Scheel M A, Kidder L E, Szilágyi B and Teukolsky S A 2013 Class. Quantum Grav. 30115001 (arXiv 1211.6079)

[156] Press W H, Teukolsky S A, Vetterling W T and Flannery B P 2007 Numerical Recipes: The Art of Scientific Computing (3rd Ed.) (New York: Cambridge University Press)

[157] Gundlach C 1998 Phys. Rev. D 57863 (arXiv gr-qc/9707050)

[158] Brown J D and York Jr J W 1993 Phys. Rev. D 471407

[159] Ashtekar A and Krishnan B 2004 Living Rev. Rel. 710 URL http://www.livingreviews.org/ lrr-2004-10

[160] Owen R 2007 Topics in Numerical Relativity: The periodic standing-wave approximation, the stability of constraints in free evolution, and the spin of dynamical black holes Ph.D. thesis California Institute of Technology URL http://resolver.caltech.edu/CaltechETD: etd-05252007-143511

[161] Pfeiffer H P, Brown D A, Kidder L E, Lindblom L, Lovelace G and Scheel M A 2007 Class. Quantum Grav. 24 S59 (arXiv gr-qc/0702106)

[162] Arnowitt R, Deser S and Misner C W 1962 The dynamics of general relativity Gravitation an introduction to current research ed Witten L (John Wiley, New York) pp 227-265 (arXiv $\mathrm{gr}-\mathrm{qc} / 0405109$

[163] York Jr J W 1979 Kinematics and dynamics of general relativity Sources of Gravitational Radiation ed Smarr L (Cambridge University Press, Cambridge) pp 83-126

[164] Beyer H R and Sarbach O 2004 Phys. Rev. D 70104004 (arXiv gr-qc/0406003)

[165] Gundlach C and Martín-García J M 2006 Phys. Rev. D 74024016 (arXiv gr-qc/0604035)

[166] Witek H, Hilditch D and Sperhake U 2011 Phys. Rev. D 83104041 (arXiv 1011.4407)

[167] Alcubierre M, Brügmann B, Diener P, Koppitz M, Pollney D, Seidel E and Takahashi R 2003 Phys. Rev. D 67084023 (arXiv gr-qc/0206072)

[168] Yo H J, Baumgarte T W and Shapiro S L 2002 Phys. Rev. D 66084026 (arXiv gr-qc/0209066)

[169] Gundlach C and Martín-García J M 2004 Phys. Rev. D 70044032 (arXiv gr-qc/0403019)

[170] Marronetti P, Tichy W, Brügmann B, González J and Sperhake U 2008 Phys. Rev. D 77064010 (arXiv 0709.2160) 
[171] van Meter J R, Baker J G, Koppitz M and Choi D I 2006 Phys. Rev. D 73124011 (arXiv gr-qc/0605030)

[172] Bowen J M and York Jr J W 1980 Phys. Rev. D 212047

[173] Brandt S and Brügmann B 1997 Phys. Rev. Lett. 783606 (arXiv gr-qc/9703066)

[174] Brügmann B, González J A, Hannam M D, Husa S, Sperhake U and Tichy W 2008 Phys. Rev. D 77024027 (arXiv gr-qc/0610128

[175] Husa S, González J A, Hannam M, Brügmann B and Sperhake U 2008 Class. Quantum Grav. $25105006(\operatorname{arXiv} 0706.0740)$

[176] Tichy W and Marronetti P 2011 Phys. Rev. D 83024012 (arXiv 1010.2936)

[177] Husa S, Hannam M, González J A, Sperhake U and Brügmann B 2008 Phys. Rev. D 77044037 (arXiv 0706.0904)

[178] Herrmann F, Hinder I, Shoemaker D, Laguna P and Matzner R A 2007 Astrophys. J. 661430 (arXiv $\mathrm{gr}-\mathrm{qc} / 0701143$ )

[179] Healy J, Herrmann F, Hinder I, Shoemaker D M, Laguna P and Matzner R A 2009 Phys. Rev. Lett. 102041101 (arXiv 0807.3292)

[180] Zlochower Y, Baker J G, Campanelli M and Lousto C O 2005 Phys. Rev. D 72024021 (arXiv $\mathrm{gr}-\mathrm{qc} / 0505055)$

[181] Mroué A H, Pfeiffer H P, Kidder L E and Teukolsky S A 2010 Phys. Rev. D 82124016 (arXiv 1004.4697 )

[182] Sperhake U 2007 Phys. Rev. D 76104015 (arXiv gr-qc/0606079)

[183] Sperhake U, Berti E, Cardoso V, González J A, Brügmann B and Ansorg M 2008 Phys. Rev. D 78064069 (arXiv 0710.3823 )

[184] Pollney D, Reisswig C, Schnetter E, Dorband N and Diener P 2011 Phys. Rev. D 83044045 (arXiv 0910.3803)

[185] Etienne Z B, Faber J A, Liu Y T, Shapiro S L, Taniguchi K and Baumgarte T W 2008 Phys. Rev. D $77084002(\operatorname{arXiv} 0712.2460)$

[186] Etienne Z B, Liu Y T, Paschalidis V and Shapiro S L 2012 Phys. Rev. D 85064029 (arXiv:1112.0568)

[187] Ansorg M, Brügmann B and Tichy W 2004 Phys. Rev. D 70064011 (arXiv gr-qc/0404056)

[188] Walther B, Brügmann B and Müller D 2009 Phys. Rev. D 79124040 (arXiv 0901.0993)

[189] Pürrer M, Husa S and Hannam M 2012 Phys. Rev. D 85124051 (arXiv 1203.4258)

[190] Hannam M, Husa S and Ó Murchadha N 2009 Phys. Rev. D 80124007 (arXiv 0908.1063)

[191] Schnetter E, Hawley S H and Hawke I 2004 Class. Quantum Grav. 211465 (arXivigr-qc/ 0310042)

[192] Brügmann B 1999 Int. J. Mod. Phys. D 885 (arXiv gr-qc/9708035)

[193] Brügmann B, Tichy W and Jansen N 2004 Phys. Rev. Lett. 92211101 (arXiv gr-qc/0312112)

[194] Goodale T, Allen G, Lanfermann G, Massó J, Radke T, Seidel E and Shalf J 2003 The Cactus framework and toolkit: Design and applications Vector and Parallel Processing VECPAR'2002, 5th International Conference, Lecture Notes in Computer Science (Berlin: Springer)

[195] Cactus computational toolkit: http://www.cactuscode.org

[196] Loffler F, Faber J, Bentivegna E, Bode T, Diener P et al. 2012 Class. Quantum Grav. 29 115001 (arXiv 1111.3344 )

[197] Einstein Toolkit: Open software for relativistic astrophysics: http://einsteintoolkit.org/

[198] Gustafsson B, Kreiss H O and Oliger J 1995 Time dependent problems and difference methods (New York: Wiley)

[199] Ajith P et al. 2007 (arXiv 0709.0093

[200] Reisswig C, Bishop N, Pollney D and Szilágyi B 2009 Phys. Rev. Lett. 103221101 (arXiv 0907. 2637)

[201] Thornburg J 1996 Phys. Rev. D 544899 (arXiv gr-qc/9508014)

[202] Thornburg J 2004 Class. Quantum Grav. 21743 (arXiv gr-qc/0306056)

[203] Campanelli M, Lousto C O, Zlochower Y, Krishnan B and Merritt D 2007 Phys. Rev. D 75 064030 (arXiv gr-qc/0612076)

[204] Sperhake U, Cardoso V, Pretorius F, Berti E, Hinderer T and Yunes N 2009 Phys. Rev. Lett. 103131102 (arXiv 0907.1252)

[205] Müller D and Brügmann B 2010 Class. Quantum Grav. 27114008 (arXiv 0912.3125)

[206] Lousto C O, Nakano H, Zlochower Y and Campanelli M 2010 Phys. Rev. D 82104057 (arXiv 1008.4360$)$

[207] Schnetter E 2010 Class. Quantum Grav. 27167001 (arXiv 1003.0859)

[208] Reisswig C, Bishop N, Pollney D and Szilágyi B 2010 Class. Quantum Grav. 27075014 (arXiv 0912.1285 
[209] Zerilli F J 1970 Phys. Rev. Lett. 24737

[210] Lousto C O 2005 Class. Quantum Grav. 22 S569 (arXiv gr-qc/0501088)

[211] Reisswig C and Pollney D 2011 Class. Quantum Grav. 28195015 (arXiv 1006.1632)

[212] Lehner L and Moreschi O M 2007 Phys. Rev. D 76124040 (arXiv 0706.1319)

[213] Berti E, Cardoso V, González J A, Sperhake U, Hannam M, Husa S and Brügmann B 2007 Phys. Rev. D 76064034 (arXiv gr-qc/0703053)

[214] Boyle M and Mroué A H 2009 Phys. Rev. D 80124045 (arXiv 0905.3177)

[215] Babiuc M C, Szilágyi B, Winicour J and Zlochower Y 2011 Phys. Rev. D 84044057 (arXiv 1011.4223$)$

[216] Boyle M, Owen R and Pfeiffer H P 2011 Phys. Rev. D 84124011 (arXiv 1110.2965

[217] Schmidt P, Hannam M and Husa S 2012 Phys. Rev. D 86104063 (arXiv 1207.3088)

[218] Boyle M 2013 Phys. Rev. D 87104006 (arXiv 1302.2919)

[219] Pan Y, Buonanno A, Fujita R, Racine E and Tagoshi H 2011 Phys. Rev. D 83064003 $(\operatorname{arXiv} 1006.0431)$

[220] Damour T, Iyer B R and Sathyaprakash B S 1998 Phys. Rev. D 57885 (arXiv gr-qc/9708034)

[221] McKechan D, Robinson C and Sathyaprakash B S 2010 Class. Quantum Grav. 27084020 (arXiv 1003.2939)

[222] Fujita R and Iyer B R 2010 Phys. Rev. D 82044051 (arXiv 1005.2266)

[223] Le Tiec A, Blanchet L and Whiting B F 2012 Phys. Rev. D 85064039 (arXiv 1111.5378)

[224] Barausse E, Buonanno A and Le Tiec A 2012 Phys. Rev. D 85064010 (arXiv 1111.5610

[225] Le Tiec A, Barausse E and Buonanno A 2012 Phys. Rev. Lett. 108131103 (arXiv 1111.5609)

[226] Akcay S, Barack L, Damour T and Sago N 2012 Phys. Rev. D 86104041 (arXiv 1209.0964)

[227] Bini D and Damour T 2012 Phys. Rev. D 86124012 (arXiv 1210.2834)

[228] Bernuzzi S, Nagar A and Zenginoğlu A 2012 Phys. Rev. D 86104038 (arXiv 1207.0769)

[229] Cutler C and Flanagan É É 1994 Phys. Rev. D 492658 (arXiv gr-qc/9402014)

[230] Droz S, Knapp D J, Poisson E and Owen B J 1999 Phys. Rev. D 59124016 (arXiv gr-qc/ 9901076)

[231] Pekowsky L, O'Shaughnessy R, Healy J and Shoemaker D 2013 Phys. Rev. D 88024040 (arXiv 1304.3176$)$

[232] Mroué A H et al. 2013 (arXiv 1304.6077)

[233] LIGO Algorithm Library Suite website: https://www.lsc-group.phys.uwm.edu/daswg/ projects/lalsuite.html

[234] IHES EOB code website: http://eob.ihes.fr/

\section{Appendix A. Initial Data Parameters}


Table A1. Initial data: mass parameters and positions. Suffixes 1 and 2 denote the two black holes. $\mu_{i}$ is the initial-data mass parameter (see code descriptions). $M$ is the sum of the Christodoulou masses of each black hole at the 'after-junk' time $t_{0} . \mathbf{x}_{i}$ is the coordinate position of each black hole. Vectors are given with subscripts $x$ and $y$ in a Cartesian coordinate system. The black holes lie in the $x y$ plane.

\begin{tabular}{|c|c|c|c|c|c|c|c|}
\hline \multirow[b]{2}{*}{ \# } & \multirow[b]{2}{*}{ Label } & \multirow[b]{2}{*}{$\mu_{1} / M$} & \multirow[b]{2}{*}{$\mu_{2} / M$} & \multicolumn{2}{|c|}{$\mathbf{x}_{1} / M$} & \multicolumn{2}{|r|}{$\mathbf{x}_{2} / M$} \\
\hline & & & & $x$ & $y$ & $x$ & $y$ \\
\hline 1 & $\mathrm{~J} 1 \mathrm{p}+49+11$ & 0.42386572 & 0.47868401 & 0 & 6.2562331 & 0 & -6.2562331 \\
\hline 2 & $\mathrm{~J} 2-15+60$ & 0.64865948 & 0.26550575 & 0 & -3.3333222 & 0 & 6.6666444 \\
\hline 3 & $\mathrm{~F} 1 \mathrm{p}+30-30$ & 0.40458816 & 0.40467792 & 0 & 5.9847297 & 0 & -5.9847297 \\
\hline 4 & $\mathrm{~F} 3+60+40$ & 0.61400511 & 0.22334067 & 0 & -2.6817824 & 0 & 8.0453472 \\
\hline 5 & $\mathrm{G} 1+60+60$ & 0.40477256 & 0.40477255 & -5.9999999 & 0 & 5.9999999 & 0 \\
\hline 6 & $\mathrm{G} 2+15-60$ & 0.65061114 & 0.26737099 & 4. & 0 & -8.0000001 & 0 \\
\hline 7 & $\mathrm{G} 2+30+00$ & 0.63208139 & 0.32260272 & 4. & 0 & -8 & 0 \\
\hline 8 & $\mathrm{G} 2+60+60$ & 0.54353789 & 0.26665158 & 3.6666667 & 0 & -7.3333333 & 0 \\
\hline 9 & R10 & 0.90392578 & 0.084911211 & -0.75029467 & 0 & 7.60393 & 0 \\
\hline 10 & L4 & 0.79227685 & 0.19127667 & 2.1865032 & 0 & -8.7460126 & 0 \\
\hline 11 & $\mathrm{~L} 3+60+00$ & 0.61425068 & 0.23827383 & -2.329625 & 0 & 6.988875 & 0 \\
\hline 12 & $\mathrm{~A} 1+30+00$ & 0.4881057 & 0.46983416 & 5.8999302 & 0 & -5.8999302 & 0 \\
\hline 13 & $\mathrm{~A} 1+60+00$ & 0.48812903 & 0.40453387 & 5.8998442 & 0 & -5.8998442 & 0 \\
\hline 14 & $\mathrm{P} 1+80-40$ & 0.45533296 & 0.30397002 & 6.2506584 & 0 & -6.2506584 & 0 \\
\hline 15 & $\mathrm{P} 1+80+40$ & 0.45524795 & 0.30394401 & 6.1759493 & 0 & -6.1759493 & 0 \\
\hline 16 & $\mathrm{~S} 1+44+44$ & 0.49986559 & 0.49986559 & 6.6810546 & 0 & -6.6810546 & 0 \\
\hline 17 & S1-44-44 & 0.49986355 & 0.49986355 & 6.6753871 & 0 & -6.6753871 & 0 \\
\hline 18 & $\mathrm{~S} 1+30+30$ & 0.49996958 & 0.49996958 & 7.4995437 & $-5.0912558 \times 10^{-8}$ & -7.4995437 & $-5.0912558 \times 10^{-8}$ \\
\hline 19 & $\mathrm{~S} 2+30+30$ & 0.66662601 & 0.333313 & 4.6301375 & 0.01288301 & -9.2949462 & 0.01288301 \\
\hline 20 & $\mathrm{~S} 3+30+30$ & 0.74995534 & 0.24998511 & 3.3712598 & 0.016546877 & -10.171299 & 0.016546877 \\
\hline 21 & S3p+00-15 & 0.74998557 & 0.24999519 & 3.5532079 & 0.016524038 & -10.706707 & 0.016524038 \\
\hline 22 & $\mathrm{~S} 1 \mathrm{p}+30+30$ & 0.49996927 & 0.49996927 & 7.359082 & 0 & -7.359082 & 0 \\
\hline 23 & S1p-30-30 & 0.50001 & 0.50001 & 8.0977416 & $1.8034293 \times 10^{-6}$ & -8.0977488 & $1.8034293 \times 10^{-6}$ \\
\hline 24 & S3-60+00 & 0.74943027 & 0.24981009 & 3.4836516 & 0.016192245 & -10.505713 & 0.016192245 \\
\hline 25 & $\mathrm{U} 1+30+00$ & 0.46984424 & 0.4881112 & -5.9 & 0 & 5.9 & 0 \\
\hline
\end{tabular}


Table A2. Initial data: velocities/momenta. Suffixes 1 and 2 denote the two black holes. $M$ is the sum of the Christodoulou masses of each black hole at the 'after-junk' time $t_{0}$. For Bowen-York configurations, the momenta $\mathbf{p}_{i}$ are given, whereas for Conformal-Thin-Sandwich configurations (those with label initial S), the velocities $\mathbf{V}_{i}$ are given. Vectors are given as $v_{x} v_{y} v_{z}$ in a Cartesian coordinate system.

\begin{tabular}{|c|c|c|c|c|c|c|c|}
\hline \multirow[b]{2}{*}{$\#$} & \multirow[b]{2}{*}{ Label } & \multicolumn{3}{|c|}{$10^{3}\left(\mathbf{p}_{1} / M \mid \mathbf{V}_{1}\right)$} & \multicolumn{3}{|c|}{$10^{3}\left(\mathbf{p}_{2} / M \mid \mathbf{V}_{2}\right)$} \\
\hline & & $x$ & $y$ & $z$ & $x$ & $y$ & $z$ \\
\hline 1 & $\mathrm{~J} 1 \mathrm{p}+49+11$ & -81.586324 & -0.44156253 & 0.38454864 & 81.586324 & 0.44156253 & -0.38454864 \\
\hline 2 & $\mathrm{~J} 2-15+60$ & 85.163716 & 0.76349079 & 0 & -85.163716 & -0.76349079 & 0 \\
\hline 3 & $\mathrm{~F} 1 \mathrm{p}+30-30$ & -85.280216 & -0.38705042 & 0 & 85.280216 & 0.38705042 & 0 \\
\hline 4 & $\mathrm{~F} 3+60+40$ & 66.395903 & 0.24967205 & 0 & -66.395903 & -0.24967205 & 0 \\
\hline 5 & $\mathrm{G} 1+60+60$ & 0.47254707 & -82.397999 & 0 & -0.47254707 & 82.397999 & 0 \\
\hline 6 & $\mathrm{G} 2+15-60$ & -0.43487079 & 75.97791 & 0 & 0.43487079 & -75.97791 & 0 \\
\hline 7 & $\mathrm{G} 2+30+00$ & -0.40590058 & 74.816237 & 0 & 0.40590058 & -74.816237 & 0 \\
\hline 8 & $\mathrm{G} 2+60+60$ & -0.49753473 & 77.378061 & 0 & 0.49753473 & -77.378061 & 0 \\
\hline 9 & R10 & 0.16787436 & -36.558416 & 0 & -0.16787436 & 36.558416 & 0 \\
\hline 10 & L4 & -0.38936512 & 58.050509 & 0 & 0.38936512 & -58.050509 & 0 \\
\hline 11 & $\mathrm{~L} 3+60+00$ & 0.48767064 & -73.103363 & 0 & -0.48767064 & 73.103363 & 0 \\
\hline 12 & $\mathrm{~A} 1+30+00$ & -0.51501591 & 85.285491 & 0 & 0.51501591 & -85.285491 & 0 \\
\hline 13 & $\mathrm{~A} 1+60+00$ & -0.51904429 & 84.698363 & 0 & 0.51904429 & -84.698363 & 0 \\
\hline 14 & $\mathrm{P} 1+80-40$ & -0.45368779 & 82.107448 & 0 & 0.45368779 & -82.107448 & 0 \\
\hline 15 & $\mathrm{P} 1+80+40$ & -0.43446276 & 80.958927 & 0 & 0.43446276 & -80.958927 & 0 \\
\hline 16 & $\mathrm{~S} 1+44+44$ & -0.33287494 & 123.67003 & 0 & 0.33287494 & -123.67003 & 0 \\
\hline 17 & S1-44-44 & -0.74710129 & 125.43959 & 0 & 0.74710129 & -125.43959 & 0 \\
\hline 18 & $\mathrm{~S} 1+30+30$ & -0.2554492 & 118.21853 & 0 & 0.2554508 & -118.21852 & 0 \\
\hline 19 & $\mathrm{~S} 2+30+30$ & -0.41767515 & 81.031813 & 0 & 0.16039033 & -162.67201 & 0 \\
\hline 20 & $\mathrm{~S} 3+30+30$ & -0.42953692 & 58.999873 & 0 & 0.13264816 & -178.00901 & 0 \\
\hline 21 & $\mathrm{~S} 3 \mathrm{p}+00-15$ & -0.45466925 & 60.404889 & 0 & 0.24265399 & -182.01832 & 0 \\
\hline 22 & $\mathrm{~S} 1 \mathrm{p}+30+30$ & 0.68885058 & 117.0166 & 0 & -0.68885058 & -117.0166 & 0 \\
\hline 23 & S1p-30-30 & -0.650836 & 115.79539 & 0 & 0.650785 & -115.79549 & 0 \\
\hline 24 & $\mathrm{~S} 3-60+00$ & -0.448744 & 61.2985 & 0 & 0.209116 & -184.862 & 0 \\
\hline 25 & $\mathrm{U} 1+30+00$ & 0.515 & -85.29 & 0 & -0.515 & 85.29 & 0 \\
\hline
\end{tabular}


Table A3. Initial data: spins. Suffixes 1 and 2 denote the two black holes. $M$ is the sum of the Christodoulou masses of each black hole at the 'after-junk' time $t_{0} . \mathbf{S}_{i}$ is the spin angular momentum of each black hole. Vectors are given as $v_{x} v_{y} v_{z}$ in a Cartesian coordinate system.

\begin{tabular}{|c|c|c|c|c|c|c|c|}
\hline \multirow[b]{2}{*}{ \# } & \multirow[b]{2}{*}{ Label } & \multicolumn{3}{|c|}{$\mathbf{S}_{1} / M^{2}$} & \multicolumn{3}{|c|}{$\mathbf{S}_{2} / M^{2}$} \\
\hline & & $x$ & $y$ & $z$ & $x$ & $y$ & $z$ \\
\hline 1 & $\mathrm{~J} 1 \mathrm{p}+49+11$ & -0.0421608 & -0.032117926 & 0.12366979 & 0.037330398 & 0.032234057 & 0.026242352 \\
\hline 2 & $\mathrm{~J} 2-15+60$ & 0 & 0 & -0.066666222 & 0 & 0 & 0.066666222 \\
\hline 3 & $\mathrm{~F} 1 \mathrm{p}+30-30$ & 0.12990416 & 0 & 0.075000204 & 0 & 0.12990416 & -0.075000204 \\
\hline 4 & $\mathrm{~F} 3+60+40$ & 0 & 0 & 0.3375 & 0 & 0 & 0.025 \\
\hline 5 & $\mathrm{G} 1+60+60$ & 0 & 0 & 0.15 & 0 & 0 & 0.15 \\
\hline 6 & $\mathrm{G} 2+15-60$ & 0 & 0 & 0.066666667 & 0 & 0 & -0.0666666667 \\
\hline 7 & $\mathrm{G} 2+30+00$ & 0 & 0 & 0.13333333 & 0 & 0 & 0 \\
\hline 8 & $\mathrm{G} 2+60+60$ & 0 & 0 & 0.26666667 & 0 & 0 & 0.066666667 \\
\hline 9 & R10 & 0 & 0 & 0 & 0 & 0 & 0 \\
\hline 10 & $\mathrm{~L} 4$ & 0 & 0 & 0 & 0 & 0 & 0 \\
\hline 11 & $\mathrm{~L} 3+60+00$ & 0 & 0 & 0.3375 & 0 & 0 & 0 \\
\hline 12 & $\mathrm{~A} 1+30+00$ & 0 & 0 & 0 & 0 & 0 & 0.074998226 \\
\hline 13 & $\mathrm{~A} 1+60+00$ & 0 & 0 & 0 & 0 & 0 & 0.14999208 \\
\hline 14 & $\mathrm{P} 1+80-40$ & 0 & 0 & -0.10002107 & 0 & 0 & 0.20004214 \\
\hline 15 & $\mathrm{P} 1+80+40$ & 0 & 0 & 0.10002107 & 0 & 0 & 0.20004214 \\
\hline 16 & $\mathrm{~S} 1+44+44$ & 0 & 0 & 0.10914509 & 0 & 0 & 0.10914509 \\
\hline 17 & S1-44-44 & 0 & 0 & -0.10940641 & 0 & 0 & -0.10940641 \\
\hline 18 & $\mathrm{~S} 1+30+30$ & 0 & 0 & 0.074990874 & 0 & 0 & 0.074990874 \\
\hline 19 & $\mathrm{~S} 2+30+30$ & 0 & 0 & 0.13331707 & 0 & 0 & 0.033329267 \\
\hline 20 & $\mathrm{~S} 3+30+30$ & 0 & 0 & 0.1687299 & 0 & 0 & 0.018747767 \\
\hline 21 & $\mathrm{~S} 3 \mathrm{p}+00-15$ & 0 & 0 & 0 & 0.016237351 & 0 & -0.0093746393 \\
\hline 22 & $\mathrm{~S} 1 \mathrm{p}+30+30$ & 0.12988784 & 0 & 0.074990781 & 0.12988784 & 0 & 0.074990781 \\
\hline 23 & S1p-30-30 & 0 & 0.12990901 & -0.075003 & 0 & 0.12990901 & -0.075003 \\
\hline 24 & S3-60+00 & 0 & 0 & -0.33698744 & 0 & 0 & 0 \\
\hline 25 & $\mathrm{U} 1+30+00$ & 0 & 0 & 0.075 & 0 & 0 & 0 \\
\hline
\end{tabular}




\section{Appendix B. Waveform Error Estimates}

Table B1. Error estimates. For each waveform, the table shows the number of cycles in the $(2,2)$ mode of $h$ between the start of the usable NR waveform and the peak of the amplitude of $h$ and the relative amplitude and absolute phase errors measured at the start of the inspiral and at the reference time shortly before the merger. The 'aligned' errors were computed using waveforms which have been aligned as described in Sec. 5.2

\begin{tabular}{lllllllll}
\hline$\#$ & Label & Cycles & $\Delta A /\left.A\right|_{\text {insp. }}$ & $\left.\Delta \phi\right|_{\text {insp. }}$ & $\Delta A /\left.A\right|_{\text {ref. }}$ & $\left.(\Delta A / A)\right|_{\text {ref. }} ^{\text {aligned }}$ & $\left.\Delta \phi\right|_{\text {ref. }}$ & $\left.\Delta \phi\right|_{\text {ref. }} ^{\text {aligned }}$ \\
\hline 1 & J1p+49+11 & 25 & 0.023 & 0.046 & 0.018 & 0.028 & 0.16 & 0.16 \\
2 & J2-15+60 & 13 & 0.0054 & 0.02 & 0.0074 & 0.0095 & 0.057 & 0.016 \\
3 & F1p+30-30 & 19 & 0.15 & 0.41 & 0.068 & 0.068 & 1.3 & 0.98 \\
4 & F3+60+40 & 24 & 0.014 & 0.064 & 0.018 & 0.012 & 0.34 & 0.2 \\
5 & G1+60+60 & 25 & 0.014 & 0.078 & 0.012 & 0.017 & 0.29 & 0.55 \\
6 & G2+15-60 & 19 & 0.013 & 0.068 & 0.04 & 0.019 & 0.67 & 0.37 \\
7 & G2+30+00 & 23 & 0.015 & 0.073 & 0.024 & 0.024 & 0.43 & 0.54 \\
8 & G2+60+60 & 22 & 0.0067 & 0.045 & 0.0048 & 0.0094 & 0.048 & 0.086 \\
9 & R10 & 13 & 0.079 & 0.3 & 0.041 & 0.019 & 0.54 & 0.16 \\
10 & L4 & 20 & 0.075 & 0.07 & 0.083 & 0.056 & 1.3 & 0.53 \\
11 & L3+60+00 & 20 & 0.041 & 0.037 & 0.17 & 0.09 & 5.5 & 2.6 \\
12 & A1+30+00 & 20 & 0.0017 & 0.011 & 0.015 & 0.009 & 0.23 & 0.07 \\
13 & A1+60+00 & 22 & 0.0038 & 0.0093 & 0.015 & 0.031 & 0.16 & 0.049 \\
14 & P1+80-40 & 24 & 0.0009 & 0.0083 & 0.035 & 0.014 & 0.87 & 0.32 \\
15 & P1+80+40 & 24 & 0.0011 & 0.0076 & 0.025 & 0.01 & 0.66 & 0.28 \\
16 & S1+44+44 & 30 & 0.0028 & 0.02 & 0.0041 & 0.0054 & 0.11 & 0.13 \\
17 & S1-44-44 & 20 & 0.0016 & 0.019 & 0.0038 & 0.0059 & 0.1 & 0.12 \\
18 & S1+30+30 & 37 & 0.0027 & 0.018 & 0.017 & 0.012 & 0.48 & 0.13 \\
19 & S2+30+30 & 34 & 0.0023 & 0.032 & 0.0032 & 0.0042 & 0.022 & 0.019 \\
20 & S3+30+30 & 37 & 0.0042 & 0.032 & 0.0049 & 0.0051 & 0.058 & 0.04 \\
21 & S3p+00-15 & 36 & 0.0036 & 0.019 & 0.02 & 0.019 & 0.39 & 0.21 \\
22 & S1p+30+30 & 35 & 0.012 & 0.1 & 0.069 & 0.023 & 1.3 & 0.26 \\
23 & S1p-30-30 & 34 & 0.0058 & 0.006 & 0.064 & 0.06 & 1.2 & 1.1 \\
24 & S3-60+00 & 27 & 0.0039 & 0.031 & 0.04 & 0.047 & 0.51 & 0.59 \\
25 & U1+30+00 & 20 & 0.04 & 0.073 & 0.063 & 0.044 & 0.99 & 0.82 \\
\hline
\end{tabular}

